\title{
ORTHODONTIC MECHANOTRANSDUCTION AND THE ROLE OF THE P2X7 RECEPTOR
}

Rodrigo F. Viecilli

Submitted to the faculty of the University Graduate School

in partial fulfillment of the requirements

for the degree

Doctor of Philosophy

in the School of Dentistry,

Indiana University

August 2009 
Accepted by the Faculty of Indiana University, in partial fulfillment of the requirements for the degree of Doctor of Philosophy.

Thomas R. Katona, Ph.D., D.M.D, Chair

Doctoral Committee

W. Eugene Roberts, Jr., D.D.S., Ph.D.

Nov. $19^{\text {th }}, 2008$

James K. Hartsfield, Jr., D.M.D., Ph.D.

Joseph Bidwell, Ph.D. 


\section{DEDICATION}

To my parents, who have supported my education. 


\section{ACKNOWLEDGMENTS}

One of the most important things I have learned during my PhD is the necessity of the existence of a working network of people that can help you achieve your goals. The more effectively this network functions, the higher the chance you will achieve the goals quickly.

I would first like to thank my parents, who have unconditionally supported my decision to abandon a profitable private practice in Orthodontics, and return to humble student conditions while aiming for a full time academic career.

I also thank my girlfriend Laura Kruter who has (almost always) been ok with my, some might say, not very sane ideas. Sometimes they do turn out fine, though (like this “ $\mathrm{PhD}$ ” one).

I can hardly think of anyone else that could have complemented my work and ideas during this Program as well as Dr. Thomas Katona. While I was passionately conditioned to an idea, he was always rational and careful. While I was sometimes furious with a shortcoming, he was always temperate and humorous. When I ran into his office with some news about a project, he would always listen and almost never seem too busy to see me. While I was working and something went wrong, he would listen and wonder with me about the "whys" and "hows", instead of telling me what to do or express disappointment. He was even subject to some of my home culinary experiments. I was extremely fortunate to find someone that thought about science the same way I did, and that actually helped me with my project instead of just telling me what to do. I felt like we worked as a team, and that helped to increase my motivation during my studies. We completed, so far, 5 papers together during my $\mathrm{PhD}$ studies. More than helping me to 
complete my work, he gave me a lifetime lesson on how I should treat my future students.

I would like to thank Dr. Charles J. Burstone, who imbued me with motivation to pursue an academic career. I don't think I would have even started my doctorate without his initial help and guidance.

The other professors who served on my advisory or research committee, also contributed in many different areas. Dr. W. Eugene Roberts (Department of Orthodontics and Orofacial Genetics) was fundamental by believing in my potential and accepting me in the Combined PhD/Orthodontics Program. When I had the engineering model ready, he pointed me in the right direction and helped me to obtain a genetic model from Dr. Charles Turner's laboratory (Department of Orthopedics), who I also must thank together with Dr. Jiliang Li and Dr. Dawei Liu. Dr. Roberts, together with Dr. Jeffrey Dean, was also decisive in making funding available to complete my research.

Dr. Jie Chen (Department of Mechanical Engineering), trusted my knowledge and guided my introduction to Finite Element Modeling. Dr. James K. Hartsfield introduced me to the contemporary world of genetics and explained the advantages of mouse models. Dr. James Baldwin was a friend for many biomechanics discussions. Dr. James Bidwell was receptive of my research idea and pointed me in the right direction by strongly recommending conventional bone label analysis in my project. Dr. Don-John Summerlin was very helpful spending a few hours with me explaining what was going on in my histological sections.

I extend thanks to Dr. Richard Gregory, who made financial support available in from second year in the Program until now. 
My thanks also go to Mrs. Patsy Dunn-Jena for assistance and guidance in several laboratory procedures. In addition, Robert Meagher must be thanked for efficiently setting up software for my research. Finally, G\&H Wire Co., which was helpful to construct the mouse springs according to my specifications. 


\begin{abstract}
Rodrigo F. Viecilli

ORTHODONTIC MECHANOTRANSDUCTION AND THE ROLE OF THE P2X7 RECEPTOR
\end{abstract}

The first part of the study describes the development of a microCT based engineering model to study orthodontic responses.

The second part investigated the relationship between orthodontic stimulus, root resorption and bone modeling. It was hypothesized that stress magnitudes are insufficient to portray the mechanical environment and explain the clinical response; directions also play a role. An idealized tooth model was constructed for finite element analysis. The principal stress magnitudes and directions were calculated in tipping and translation. It was concluded that within the same region of root, PDL and bone, there can be compression in one structure, tension in another. At a given point in a structure, compression and tension can coexist in different directions. Magnitudes of compression or tension are typically different in different directions. Previously published data presenting only stress magnitude plots can be confusing, perhaps impossible to understand and/or correlate with biological responses. To avoid ambiguities, a reference to a principal stress should include its predominant direction. Combined stress magnitude/direction results suggest that the PDL is the initiator of mechanotransduction.

The third part of this project tested the role of the P2X7 receptor in the dentoalveolar morphology of C57B/6 mice. P2X7R KO (knockout) mice were compared to $\mathrm{C} 57 \mathrm{~B} / 6 \mathrm{WT}$ to identify differences in a maxillary molar and bone. Tooth dimensions 
were measured and 3D bone morphometry was conducted. No statistically significant differences were found between the two mouse types. $P 2 X 7 R$ does not have a major effect on alveolar bone or tooth morphology.

The final part examines the role of the P2X7 receptor in a controlled biomechanical model. Orthodontic mechanotransduction was compared in wild-type (WT) and P2X7R knock-out (KO) mice. Using Finite Element Analysis, mouse mechanics were scaled to produce typical human stress levels. Relationships between the biological responses and the calculated stresses were statistically tested and compared. There were direct relationships between certain stress magnitudes and root resorption and bone formation. Hyalinization and root and bone resorption were different in WT and KO. Orthodontic responses are related to the principal stress patterns in the PDL and the P2X7 receptor plays a significant role in their mechanotransduction.

Thomas R. Katona, Ph.D., D.M.D, Chair 


\section{TABLE OF CONTENTS}

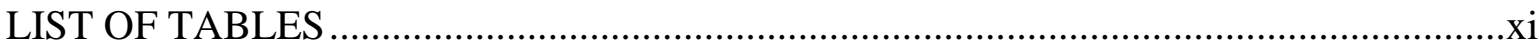

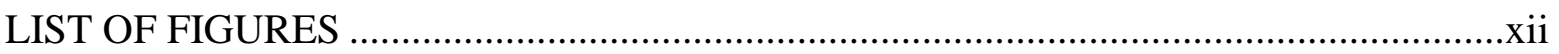

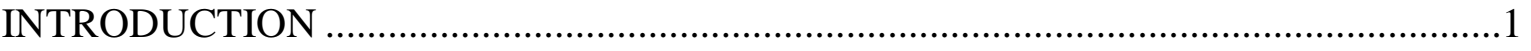

Orthodontic Tooth Movement, Bone Response and Root Resorption ........................1

Background ............................................................................................

Malocclusion...............................................................................................

Mechanical variables in orthodontic tooth movement and ERR .....................4

Orthodontic mechanotransduction ...............................................................

Biological factors in rate of tooth movement and ERR.................................14

Damage-associated molecular patterns/Alarmins ............................................17

HMGB1 and Bone ................................................................................19

P2X7 receptor: a possible mediator of orthodontic mechanotransduction ....20

OPTIMIZATION OF MICROCT DATA FOR MODELLING OF DENTAL

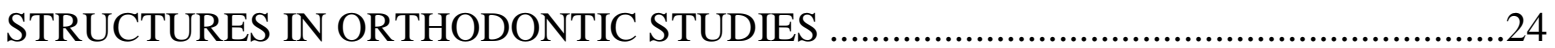

THE THREE-DIMENSIONAL MECHANICAL ENVIRONMENT OF

ORTHODONTIC TOOTH MOVEMENT AND ROOT RESORPTION ...............................37

COMPARISON OF DENTOALVEOLAR MORPHOLOGY IN C57B/6 AND P2X7R

KO MICE FOR THE DEVELOPMENT OF BIOMECHANICAL ORTHODONTIC

MODELS 


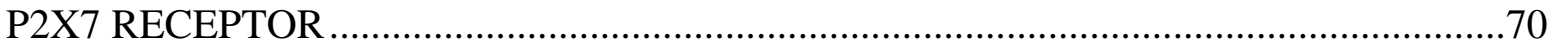

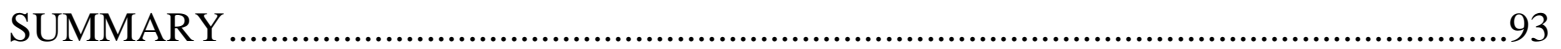

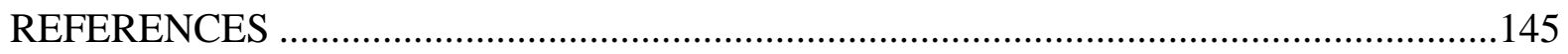

CURRICULUM VITAE 


\section{LIST OF TABLES}

I Bone morphometry data from the sphere taken from the C57B6 mouse. Standard abbreviations are used.

II Comparison of maxillary molar measurements in WT and KO mice .98

III Comparison of bone 3D morphometric parameters. BMD= bone mineral density; $\mathrm{BV} / \mathrm{TV}=$ bone volume fraction; $\mathrm{BS} / \mathrm{BV}=$ bone surface-volume ratio; $\mathrm{Tr}$.Th= trabecular thickness; Tr.Sp= trabecular separation; Tr.N= trabecular number; Tr.Pf= trabecular pattern factor; $\mathrm{SMI}=$ structure model index; $\mathrm{DA}=$ degree of anisotropy; $\mathrm{FD}=$ fractal dimension. .99

IV Comparison of bone morphometry parameters of alveolar trabecular bone before and after application of orthodontic force in $\mathrm{WT}$ and $\mathrm{KO}$ mice. $\mathrm{BMD}=$ bone mineral density; $\mathrm{BV} / \mathrm{TV}=$ bone volume fraction; $\mathrm{BS} / \mathrm{BV}=$ bone surface-volume ratio; $\mathrm{Tr}$. Th= trabecular thickness; Tr.Sp= trabecular separation; Tr.N= trabecular number; Tr.Pf= trabecular pattern factor; SMI= structure model index; DA= degree of anisotropy; $\mathrm{FD}=$ fractal dimension .100

V Comparison of alveolar trabecular bone morphometry after force application in WT and $\mathrm{KO}$ mice. $\mathrm{BMD}=$ bone mineral density; $\mathrm{BV} / \mathrm{TV}=$ bone volume fraction; $\mathrm{BS} / \mathrm{BV}=$ bone surface-volume ratio; Tr.Th= trabecular thickness; Tr.Sp= trabecular separation; Tr.N= trabecular number; Tr.Pf= trabecular pattern factor; $\mathrm{SMI}=$ structure model index; $\mathrm{DA}=$ degree of anisotropy; FD= fractal dimension. 101 


\section{LIST OF FIGURES}

Figure 1. Fatigue experiment diagram: the apparatus for loading of the dentoalveolar structures of a mandibular lower incisor is illustrated 102

Figure 2. The newly developed NiTi coilspring and its insertion procedure 103

Figure 3. Sections scanned without (left) and with (right) median filter. Note the sharpness and detail of the left image in which random movement of 100 lines and averaging of 8 frames were used to remove noise instead of the Median Filter

Figure 4. Alignment (wing) artefacts (arrows) on the mesial root of the mouse first molar. .105

Figure 5. Alignment tool, as used for alignment (left) and with separated cylinders for specimen scanning (right) 106

Figure 6. A difference in the object magnification from 0 to 180 degrees scans. The higher the object width $b$ and the higher the magnification, increased by decreasing a, the higher the discrepancy c....

Figure 7. Segmentation of bone, periodontal ligament and tooth into different masks before and after 3D rendering 108

Figure 8. Bone plane cut to show the $0.5 \mathrm{~mm}$ diameter spherical sample from alveolar bone between the roots. Transparency was toggled to allow interior visualization .109

Figure 9. Dog incisor and mouse molar root rendered with maximum accuracy. A close up to visualize Retzius striae, cementum-enamel junction and root surface roughness in the dog tooth is also included .110 
Figure 10. Examples of solid models generated after editing the stl files. Smoothing was performed to allow quality meshing, but the main features of the original scan were maintained

Figure 11. Diagram of contemporary orthodontic biomechanics

Figure 12. A, General state of stress; B, Principal stresses in the principal (x’, y’, z')

directions

Figure 13. A, CAD model showing internal morphology; B, FE model and coordinate system

Figure 14. A, Exaggerated (10x) displaced positions of the root. B, Deformed root shapes. C, Exaggerated (x5000) total tooth displacement in translation. The scale is in $\mathrm{mm}$ 115

Figure 15. Exaggerated 3-D occlusal view of socket deformations resulting from tooth translation. The final position is the colored solid and the initial position is the element skeleton .116

Figure 16. Tooth translation associated principal stresses in the root: A, distal side S1 (S3 is almost zero;) B, mesial side S3 (S1 is almost zero;) C, distal side S2;

$\mathrm{D}$, mesial side S2 117

Figure 17. Tooth tipping associated principal stresses in the root: A, distal side S3

( $\mathrm{S} 1$ is almost zero); $\mathrm{B}$, mesial side $\mathrm{S} 1$ ( $\mathrm{S} 3$ is almost zero); C, distal side S2;

$\mathrm{D}$, mesial side $\mathrm{S} 2$

Figure 18. Tooth translation associated principal stresses and their components on the distal side of the PDL: A, S1; B, S2; C, S3. D, buccal view of PDL principal stresses in the apical $\sim 1 / 3$ of the root during translation (left side is the distal side) 119 
Figure 19. Tooth tipping associated principal stresses and their components on the distal side of the PDL: A, S1; B, S2; C, S3

Figure 20. Three-dimensional magnitude plot of tooth translation associated principal stresses in bone. A, S1; B, S2; C, S3

Figure 21. 3-D direction plots of tooth translation associated principal stresses in bone, A, distal side (cuspid socket is on the right) and B, mesial side (cuspid socket is on the left;) Polar plots at the cervical region of the bone for C, S1 and D, S3. S2 is not shown because it is very small

Figure 22. As in Figure 10 except for tooth tipping.

Figure 23. As in Figure 11 except for tooth tipping.

Figure 24. Typical histological section, focusing on the root and PDL. Bone and tooth root are stained blue, and PDL is stained pink. There were no noticeable differences between WT and KO sections 126

Figure 25. Surface finite element model of a representative specimen showing highly organic internal structure, even after smoothing of tissues. Yellow= tooth, blue= bone, red $=$ PDL

Figure 26. Resulting solid 3D finite element model obtained in ANSYS .128

Figure 27. A, Segmentation and B, 3D reconstruction of the mouse molar. C, Isolated trabecular bone sphere for bone morphometry. D, Finite element model of the mouse molar. 129

Figure 28. A, CAD model of the segment, and a cut showing the periodontal ligament, adjacent premolar socket and lateral incisor root. B, Finite element model 130 
Figure 29. A Mouse spring insertion procedure with custom prop. B, Spring in place. C, Sagittal and D, occlusal views of the line of action of the force.

Figure 30. A, Occlusal view of idealized human canine PDL stress directions during tipping. The small red and white arrows represent maximum compressive and tensile stress directions, respectively. Note that the line of action of the force (large white arrow) and the maximum PDL stress directions are coincident with each other. B, and C, Apical views of the mouse PDL stress directions during tipping, with the correspondent root identification ( $\mathrm{DB}=$ disto-buccal, $\mathrm{DP}=$ disto-palatal and $\mathrm{M}=$ =mesial). Histological cutting plane for ERR analysis (dashed black line in B, C, and D follows the maximum compression PDL stress direction (red arrows), not the line of action of the force (white arrow in D.) .132

Figure 31. Static sections of the 3D FE model showing calculated initial (contour) and final (solid) positions of the teeth on the A, ERR analysis section plane and B, bone formation analysis section plane. Because the sections followed stress directions, the tooth has negligible out-of- plane movement. This is evidenced by little change observed in tooth and root shape between the initial and final positions in these planes

Figure 32. Method to relate FE-computed maximum PDL tensile stresses (left) to histologically measured interlabel bone distances (right.) In-plane maximum tensile stresses, acting horizontally, decrease from region 8 to 5. Note that the pattern of PDL deformation was correctly predicted by the FEA. In bone measurements of the picture, $\mathrm{d} 3$ would be discarded because it is in a remodeling cavity, and only $\mathrm{d} 1$ and $\mathrm{d} 2$ would be averaged. The scale shows the maximum tensile stress values in Pascals. .134

Figure 33. Comparison of tooth hyalinization scores in treated KO and WT mice 135 
Figure 34. Comparison of ERR proportion score by region (Fig. 11) within each strain of treated mice. Significant differences in the WT and KO were found between regions 1 and $2(\mathrm{p}=0.002, \mathrm{p}=0.001)$, and 1 and $3(\mathrm{p}<0.001, \mathrm{p}=0.002)$.

Figure 35. Comparison of ERR proportion score by type in the region of high stress $(\mathrm{p}<0.02)$

Figure 36. Illustration of microCT root resorption detection. A, Histological section of the DB root of a KO force group mouse. B, MicroCT reconstructed section of the same region and C, its segmentation. Such segmentation of all sections allows 3D rendering of the structures and volume estimation. D, Visualization of resorption cavities (c) and the area of hyalinized PDL tissue (Hz)

Figure 37. Tooth volume by type in control and force groups. A significant difference was found only after force application, confirming that KO has more $\operatorname{ERR}(p=0.002)$ 139 Figure 38. Example sections of WT (A and C) and KO (B and D) histology. C and D are magnified high stress regions of A and B, respectively. Note the increased presence of neutrophils (lobular shaped nuclei) in the WT section. .140

Figure 39. Example section of a labeled control WT disto-palatal root. Note normal bone apposition around blood vessels. New bone is also observed on the palatal periosteal surface and near the apex, suggesting slight eruption during the experimental period. Due to this interference, the apical regions (2 apical region 5 points and region 6) were eliminated from linear regression analysis in the treated mice. 
Figure 40. Linear regression plots of the natural logarithm of maximum principal stress [ln (Stress)] vs. Bone Interlabel Distance in the DP root of WT and KO mice. The outer lines in each regression are the limits of the 95\% confidence interval 142

Figure 41. A, Histological section of a KO mouse. Large area of hyalinized tissue remains on the compression side of the $\mathrm{DB}$ root. $\mathrm{B}$, Corresponding section of the $\mathrm{FE}$ model. Note that the FEA correctly predicted the deformation of the PDL. Hyalinization occurs at PDL stress levels $>\sim 10 \mathrm{KPa}$ .143

Figure 42. Structures around the high-tension PDL of DB root. Note stretched fibroblasts and the absence of bone formation and inflammatory cells within the socket. Bone formation, however, is prominent on the periosteum of the buccal surface.

(1) Root. (2) PDL with stretched fibroblasts. (3) Buccal alveolar wall. (4) Osteoid.

(5) Osteoblasts. (6) Trapped osteoblast that would become an osteocyte.

(7) Cervical area where once there was resorption, and now there is formation .144 


\section{INTRODUCTION}

\section{Orthodontic Tooth Movement, Bone Response and Root Resorption}

\section{Background}

The identification of variables associated with orthodontic ERR (external root resorption) and rate of tooth movement has been a subject of research for many decades. This problem has been examined in cellular, animal and clinical experimental models. Although the literature is abundant, results from each model have been contradictory. Clinical studies, despite providing the most realistic orthodontic model possible, are often limited to 2D radiographic data. The existence of a large range of morphological variability in dento-alveolar structures requires large numbers of subjects to maintain statistical power, causing these types of studies to be expensive and laborious (Chan \& Darendeliler, 2004; Darendeliler et al., 2004). Given that the biomechanical relationships between many of the variables are not known, or sometimes not considered, the existence of controversial results is not surprising.Traditional animal models allow in-depth analysis of the physiology with histology. However, they are typically compromised by the same difficulties: 2D data and problems with ERR and bone formation assessment techniques.

Inbred strains of mice (Thyagarajan et al., 2003), where individual variability is practically eliminated, have recently been explored in orthodontic studies (Al-Qawasmi et al., 2004; Al-Qawasmi et al., 2006; Ignelzi et al., 1995; Oshiro et al., 2002). With the development of genetic engineering, it has become possible to analyze the effects of a single biological variable (encoded by a gene) in orthodontic responses. However, it is still a challenge to accurately quantify 3D experimental variables such as root resorption 
and bone formation. It is known that the mechanical stimuli for orthodontic responses are the 3D distributed stresses/strains on the root and bone-PDL (periodontal ligament) interface (Andersen et al., 1991a; Andersen, Pedersen \& Melsen, 1991b; Cattaneo, Dalstra \& Melsen, 2005; Davidovitch, 1991; Fukui, 1993; Jones et al., 2001; Katona et al., 1995; Kawarizadeh et al., 2003a; Kawarizadeh et al., 2004; Krishnan \& Davidovitch, 2006; Melsen, 1999; Melsen, 2001; Middleton, Jones \& Wilson, 1996; Provatidis, 2002; Roberts, Goodwin \& Heiner, 1981; Rygh, 1976; Toms et al., 2002; Wright \& Yettram, 1979). Histology provides only 2D data and the results greatly depend on the choice of the representative sections, as well as choice of method of root resorption assessment. Because the tooth root is a 3D object, it is technically difficult to choose sections according to a method that is biomechanically consistent (i.e., to cut the dento-alveolar complex through areas of similar stresses and strains). Moreover, if bone formation is studied, the use of traditional bone labels for quantification requires thick mineralized histological sections, which further increase the above mentioned complications. It has been argued that the methodology for this type of research needs to be significantly improved (Chan \& Darendeliler, 2005; Chan \& Darendeliler, 2006; Chan \& Darendeliler, 2004; Chan et al., 2004a; Chan et al., 2004b; Chan, Petocz \& Darendeliler, 2005; Darendeliler et al., 2004).

The methods developed in this study can be used as tools for 3D-based quantification of biomechanical variables such as PDL stress, bone microstructure, ERR and bone formation in orthodontic animal studies. The experiments with the $P 2 X 7 R$ mice serve as the first example of application of this model, as well as provide animal evidence 
for the role of this receptor in orthodontics associated bone modeling and remodeling, and ERR.

\section{Malocclusion}

Malocclusion is a common problem. A survey in the 1960's showed that $60 \%$ of children between 6 and 11 years old and more than 83\% of teenagers in the 12-17 age interval had crowding and misalignments (Proffit \& Fields, 2000). Such orthodontic problems may lead to psychosocial disorders, muscular stress and speech difficulties.

The large prevalence of malocclusion implies that side effects of orthodontic treatment, such as ERR, are also a large population problem (Jakobsson \& Lind, 1973; Lupi, Handelman \& Sadowsky, 1996; Mirabella \& Artun, 1995a). For instance, one study showed that 50 out of 54 maxillary central incisors of orthodontic patients had radiographic evidence of ERR (McLaughlin, 1964), 25\% had more than 3mm of resorption (Hollender, Ronnerman \& Thilander, 1980) and 5\% had more than 5mm (Copeland \& Green, 1986). Histological studies often reveal a larger prevalence and extent of ERR than 2D radiographic studies, because resorption on buccal and lingual faces of the root cannot be seen on the radiograph (Kurol, Owman-Moll \& Lundgren, 1996). This type of side effect can result in legal complications for orthodontists, which in itself may contribute to increases in malpractice insurance costs (Brezniak \& Wasserstein, 1993a; Brezniak \& Wasserstein, 1993b; Brezniak \& Wasserstein, 2002b). Patients who undergo significant ERR partially lose the PDL and bone support, which can result in immediate deleterious consequences. In addition, in the event of establishment of a periodontal disease, less tissue destruction is necessary to increase tooth mobility and in the ultimate tooth loss. The possibility of genetic predisposition of 
ERR has accumulated a large amount of evidence in the last decade. Family clustering (Harris, Kineret \& Tolley, 1997; Newman, 1975), influence of ethnicity (Sameshima \& Sinclair, 2001), heritability studies (Harris et al., 1997; Ngan et al., 2004), and clinical association studies (Al-Qawasmi et al., 2003a) have provided extensive evidence for the existence of genetic predisposition to ERR. The identification of genes that mediate the ERR process will possibly become a future prevention strategy. In addition, it may also lead to technologies to modulate its effects directly during treatment.

The increasing demand for orthodontic treatment in the US as a result of population growth, has not been compensated by an increase in the number of orthodontists (Waldman, 1998), possibly because of the reduced number of orthodontic residency programs and the high rate of retiring orthodontists. A possible solution to this problem is to discover methods to enhance orthodontic treatment velocity. Several attempts to accomplish this have been made; however, they are often associated with traumatic surgical procedures, or increased side effects such as ERR and pain. To stimulate basic research that focuses on the understanding of the normal physiology of mechanical and molecular mechanisms that control orthodontic mechanotransduction is probably a good long-term strategy to open new possibilities for breakthroughs in this area.

\section{Mechanical variables in orthodontic tooth movement and ERR}

Orthodontic tooth movement is ultimately a result of bone adaptation to stresses/strains induced by forces and/or moments in the bone and PDL (Burstone \& Pryputniewicz, 1980; Choy et al., 2000; Christiansen \& Burstone, 1969; Fukui, 1993; Katona et al., 1995; Kawarizadeh et al., 2003a; Kawarizadeh et al., 2004; Melsen, 1999; 
Melsen, 2001; Middleton et al., 1996; Nagerl et al., 1991; Smith \& Burstone, 1984;

Tanne, Burstone \& Sakuda, 1989a; Tanne et al., 1989b; Tanne, Sakuda \& Burstone, 1987; Toms et al., 2002; van Steenbergen et al., 2005a; van Steenbergen et al., 2005b; Vanden Bulcke et al., 1987; Vanden Bulcke et al., 1986). The changes in the mechanical environment that surrounds the tooth depend not only on the force system, but also on the morphology of the root and alveolar bone (Burstone \& Pryputniewicz, 1980; Choy et al., 2000; Nagerl et al., 1991; Tanne et al., 1989a; Tanne et al., 1991). Because the stress magnitude is inversely related to the extension of the loaded area, it is natural that different regions surrounding the root will withstand more stresses than others. In addition, localized irregularities will act as stress concentrators, because of their relative small surface. Studies that examined ERR damage to the surface of the root revealed that this damage is concentrated in areas of PDL hyalinization (Kurol \& Owman-Moll, 1998; Miyoshi et al., 2001; Nakamura, Sahara \& Deguchi, 2001; Ramanathan \& Hofman, 2006; Rygh, 1974; von Bohl et al., 2004), which, in turn, are result of compressive strains. A recent study that used software to convert 2D scanning microscopy images of ERR in 3D volumetric data showed that there are significant and much more ERR in areas of compression than tension, and that the extent of ERR increases with the force magnitude (Chan \& Darendeliler, 2006). This study used the difference in grayscale caused by the topology of the root surface, obtained with scanning electron microscopy (2D image), multiplied by the area of the crater to perform software conversion from 2D to 3D data ( 15\% error rate). It was the first attempt to explore the third dimension in ERR. However, an experiment that provides true 3D measurements of volume for either ERR or bone has never been published. 
Another important aspect of this biomechanics is the possibility of bending of the root (Graber, Vanarsdall \& Vig, 2005), especially significant if we consider the high loads exerted by the muscles involved in mastication. In addition, when the teeth move, there may be a premature contact situation, which can result in loading of a single tooth with forces that were physiologically appropriate for the entire dentition. Although this particular aspect of ERR is still under investigation, it is probably natural that under these circumstances, where load is very high, the pattern of damage distribution along the root could be inconsistent with strains in the PDL. However, most of the evidence tends to show correlation of ERR with areas of PDL compression and not root bending, and the effect of root bending on root resorption still needs to be further investigated.

Studies have tried to assess parameters that affect the distribution of stresses and strains, such as root shape and length. Some studies have found that shorter roots undergo more ERR (Goldson \& Henrikson, 1975; Harris et al., 1997; Jakobsson \& Lind, 1973; Newman, 1975; Taithongchai, Sookkorn \& Killiany, 1996; Thongudomporn \& Freer, 1998), others found no difference (Goldin, 1989; Levander \& Malmgren, 1988), and others found the opposite (Mirabella \& Artun, 1995b; Sameshima \& Sinclair, 2001). A likely mechanical explanation for this controversy is that length alone is a very poor indicator of the stresses/strains on the apical region of the root. If a root is longer, it can so be by changing its shape in different ways, also changing the surface area distribution along the root and the position of the center of resistance. In this situation, it is impossible to predict stresses and strains in a generalized manner. On the other hand, clinically determined abnormalities in root shape such as pipette-shaped and apically bent roots denote a relatively large portion of the root with a small surface area, which will typically 
develop significant stress concentration if compared to a normal root. Typically, the literature is in agreement that abnormal shapes are correlated to an increase in ERR (Hartsfield, Everett \& Al-Qawasmi, 2004; Kjaer, 1995; Levander \& Malmgren, 1988; McFadden et al., 1989; Mirabella \& Artun, 1995b; Newman, 1975; Sameshima \& Sinclair, 2001; Thongudomporn \& Freer, 1998).

A good experimental model for comparison of biological factors that influence ERR needs to be able to control and somehow quantify the parameters that determine stresses and strains which act along the dento-alveolar complex. To do this, it is necessary to control root morphology, natural occlusion, and to apply a reproducible and accurate force system. This type of variable control not only increases the statistical power of the study by decreasing in-group result variability, but also increases the strength of the evidence provided by avoiding confounders. For instance, the investigation of the effect of a biological variable, such as the influence of a gene that encodes a growth factor in inflammation associated with ERR, could be problematic if this growth factor previously affected the morphology of the root during tooth formation. If root morphology differences are not quantified, the results of the study could reflect a change in the mechanical environment rather than reveal the direct role of this particular biological variable in inflammation. Moreover, depending on the region of the root that is analyzed in each study, studies on the same growth factor could provide opposite results, because different root regions in different roots would have unpredictable comparison values. 


\section{Orthodontic mechanotransduction}

Tooth movement occurs after mechanical stimuli, from occlusion and/or orthodontics are transduced into biological signals that ultimately result in catabolic and anabolic bone modeling, and a local increase in the bone remodeling rate (Roberts, 1999; Verna \& Melsen, 2003; Verna, Zaffe \& Siciliani, 1999). The rate limiting step in this process is catabolic modeling, because after PDL equilibrium, tooth displacement is primarily limited by bone resorption (Roberts, 2004).

The process of orthodontic mechanotransduction, or how the bone, root surface and/or PDL react to the stresses and strains through biological signals, is still far from clear. The first attempts to understand this process were made with histological research by Sandstedt, Oppenheim and Schwartz, which led to the so called "pressure-tension” theory (Krishnan \& Davidovitch, 2006). The theory basically states that in the pressure side cell replication decreases due to vascular constriction, whereas in the tension side the opposite happens due to stretching of PDL fibers. This concept was further developed by Schwartz, who tried to correlate the applied force with the capillary bed blood pressure, stating that forces should not overcome this pressure on the root surface (Schwarz, 1932). If they do, tissue necrosis would occur (PDL hyalinization). Reitan defined hyalinization as areas where normal PDL structure has been disrupted: fiber organization was lost and no cells are observed (Reitan, 1994). He found that hyalinization occurred more during tipping than during translation. These studies were the first to conclude that the tissue degeneration and rate of tooth movement were correlated to a force per unit area (stress). The bone in compression areas could be resorbed directly, by osteoclasts lining up on the bone-PDL interface, or indirectly, on the underside of bone beyond necrotic tissue. Areas 
of hyalinization produced undermining resorption, characterized by bone resorption adjacent to the necrotic area. Melsen hypothesized that indirect resorption, unlike direct resorption, is not directly induced by the load, but by the inflammation that follows the presence of necrotic tissue itself (Melsen, 1999). FEA simulations seem to corroborate that the correlation of principal or linear stresses in the PDL coincide with the actual movement tendency and cell response (Katona et al., 1995; Kawarizadeh et al., 2003a; Kawarizadeh et al., 2004). In addition, analysis of location of ERR sites were visually compatible with these areas of high stresses and strains related to tension and mainly compression (Chan \& Darendeliler, 2005; Chan \& Darendeliler, 2006). However, a systematic study that examines the 3D distribution of EARR sites in direct comparison to bone or PDL stimulation is still lacking.

An alternative theory of how tissues react to the mechanical stimulus is based on bone bending, initially suggested by Farrar (Krishnan \& Davidovitch, 2006). Epker and Frost are responsible for a well-known version of this theory, which states that the alveolar bone wall at the tension side bends decreasing its radius of curvature, causing bone compression which results in apposition and resorption (as a result of a regional acceleratory phenomenon) (Epker \& Frost, 1965). Experiments in dog mandibles also showed that in areas of PDL tension the bone assumes a concave shape, whereas in compression areas it tends to assume a convex shape (Bassett \& Becker, 1962; Bassett, Pawluk \& Becker, 1964; Krishnan \& Davidovitch, 2006; Zengo et al., 1974; Zengo, Pawluk \& Bassett, 1973). According to this theory, the resulting apposition and resorption processes in orthodontics would then be consistent with known orthopedic behavior of long bones. The problem of this theory seems to be the lack of extensive 
literature showing correlation of the strains in the bone with the actual cellular response, measured by labeling bone or cell activity markers. The high strain levels that lead to formation in long bones have not been matched by many of the FE simulations because orthodontic tooth movement happens even with extremely small loads. Recently, a purely mechanical finite element simulation was published to try to correlate this theory with the orthodontic response, arguing that the bone is being continuously bent by pre-tension in the PDL fibers in strain levels that match the "maintenance" strain levels for bone structure (Cattaneo et al., 2005). The application of compression in these areas would lead to "disuse", or "lack of tension" and, when combined with RAP, bone resorption. In the tension side, the load would tend to add to the pre-existent tension and thus induce bone apposition. Although the FEA could show consistent results with this theory, the gap of this model is the lack of data regarding the magnitude of pre-tension in the PDL fibers, so that strain values cannot be compared directly with orthopedic standards for disuse, maintenance, formation and crack formation levels.

Until now, two animal studies have tried to correlate biological responses in terms of bone formation with mechanical stimulus. In one of them, a 2D finite element analysis (FEA) of rat tooth movement was used for 2D histological correlation of osteoblast activity (Katona et al., 1995). The other performed a 3D FEA and correlated strains with osteoclast activity in 2D histological sections (Kawarizadeh et al., 2003a; Kawarizadeh et al., 2004). The main limitation of these studies is the lack of 3D biological data on bone formation and/or cell activity. The results are also limited by the technical difficulties involved in calibration of the FE model with the actual in vivo tissue behavior, lack of 
simulation of the direction effect of PDL fibers, and the natural limitations of the use of a computer model rather than an in vivo simulation.

From a molecular perspective, bone mechanotransduction has been associated mainly with fluid flow stimulation of osteocytes (Roberts, 2004). A comprehensive review by Robling, Castillo and Turner (Robling, Castillo \& Turner, 2006b) summarized the known mechanisms of bone mechanotransduction: when the bone is loaded, bending causes fluid movement in the canalicular network, which leads to shear stress on integrin and actin molecules mainly on the cell surface of osteocytes. This effect has been also observed in other bone cells; however, because the proportion between osteocytes and other bone cells is approximately 20:1, it is believed that osteocytes probably have the main role in this process. Fluid shear enhances ATP release and causes an influx of $\mathrm{Ca}^{2+}$ via voltage-sensitive channels. ATP then binds to P2Y (g-protein coupled) or P2X (ligand-gated ion channel) receptors. The P2Y G protein pathway leads to $\mathrm{Ca}^{2+}$ release. ATP binds to P2X7 receptor increasing pore formation and causing the release of PGE2, which in turn binds to EP (PGE receptor) stimulating bone formation through a pathway that is still unknown. PTH seems to have a role in this process to increase PGE2 levels, also through a pathway that is still unknown. Typically PTH tends to increase bone resorption, but intermittent PTH hormone injections induce formation. The anabolic action involves hormone regulation of apoptosis, proliferation, and differentiation of cells of the osteoblast lineage. In addition, there is regulation of remodeling, and impact on systemic factors such as FGF2, vitamin D and others (Jilka, 2007; Goltzman, 2008). There is evidence that wnt (wingless-type MMTV integration site family member) signaling through the lrp5 receptor (LDL-receptor related protein 5), which acts through 
beta-catenin translocation to the nucleus to modify gene expression, also has a role in bone formation. Pressure on the marrow cavity or fluid stress on marrow cells may stimulate NO synthase activity and NO release, which is a strong inhibitor of bone resorption and probably decreases RANK-L expression and increases OPG production, ultimately inhibiting osteoclast differentiation. PGE2 and PTH (parathyroid hormone) apparently maintain a close relationship in mechanotransduction.

PDL stress/strain is also a strong candidate for a role in orthodontic mechanotransduction. The strain, which initially acts on the extracellular matrix, is transduced to the cytoskeleton through cell surface proteins, such as actin (microfilaments) and integrins (which connect the cell to the extracellular matrix) (Krishnan \& Davidovitch, 2006). The strains can induce reorganization of the cytoskeleton, secretion of cytokines, ribosomal activation and gene transcription. Studies in fibroblasts from the PDL showed that tension stress decreases M-CSF production, a factor important for osteoclast differentiation (M-CSF knockout mice have osteopetrosis (Yoshida et al., 1990)). This would cause a net decrease in bone resorption that could favor the anabolic component of remodeling in the tension side of the PDL. Another in vitro study on osteoblast-like cells showed that IL-1 $\beta$ production is inhibited under tensile strains of lower magnitude, again contributing to bone formation in areas subject to tension (Agarwal et al., 2003; Long et al., 2002). According to the same studies, this cytokine leads to a decrease of PGE2 production in fibroblasts under low-magnitude tensile stress. Unlike bone mechanotransduction mechanisms, PDL transduction mechanisms seem to connect IL-1 $\beta$ and PGE2 with bone resorption (Agarwal et al., 1998; Agarwal et al., 2003; Brady et al., 1998; Long et al., 2002; Saito et al., 1991b). 
Studies on the role of ATP as a signaling molecule also open the possibility of purine receptor activation in fibroblasts, monocytes and macrophages, which could potentially lead to release of inflammatory mediators. This particular aspect of transduction will be discussed in the $\mathrm{P} 2 \mathrm{X} 7$ receptor section.

Although defenders of both tooth movement theories have tried to think in separate ways, evidence supporting both sides raises the possibility that orthodontic mechanotransduction happens as a simultaneous response to bone loading (with the resulting bone mechanotransduction pathways), and PDL loading and damage (with the resultant inflammatory pathways). Orthodontics has taken benefit from the results of general bone biology and immunology research for many decades. However, because of the specific physiology of the bone-PDL interface, the mechanisms can be more intricate and complex, and theoretically need much more effort to be unveiled. On the other hand, because orthodontics can take advantage of previous research results from bone biology and immunology, experimental models can be planned at relatively advanced levels of investigation. It is hard, or maybe impossible, to perform experiments where bone, PDL and tooth root cells maintain a relationship close as in live animals. Animal models are probably the only comprehensive way to test the roles of different molecular mechanisms in orthodontic mechanotransduction.

The transduction mechanisms for root resorption are basically unknown. Evidence shows that compressive zones, associated with hyalinization, undergo much more resorption than tensile areas. Root resorption continues even after the force application is stopped, but halts after the PDL structure is restored (Brudvik \& Rygh, 1995a; Brudvik \& Rygh, 1995b). This opens the possibility for the hypothesis that normal PDL physiology, 
or PDL tension, are necessary to maintain balance between repair and resorption. From the molecular perspective, one study showed that calcitonin, a known inhibitor of osteoclast activity, also inhibits odontoclast activity, but the signaling pathway uses protein kinase A (PKA), which is different from osteoclast signaling. This result also corroborates the hypothesis that mechanotransduction in orthodontics may be a result of complex or specific molecular pathways.

\section{Biological factors in rate of tooth movement and ERR}

Rate of orthodontic tooth movement and ERR are influenced by a wide range of molecule types. Cytokines, prostaglandins, nitric oxide, hormones, the biologically active form of Vitamin D and growth factors are widely accepted examples (Krishnan \& Davidovitch, 2006; Roberts, 2004). Each molecular category acts on different levels of the response. For many one of these, there are genes that may encode molecules which perform their actions with normal, increased or decreased activity. This is part of the physiological basis for diversity in orthodontic responses that needs to be unveiled, to determine to which extent the response can be modified with treatment.

Levels of cytokines such as IL1- $\beta$ and TNF- $\alpha$ have been associated with enhancement of tooth movement (Agarwal et al., 1998; Davidovitch et al., 1988; Hou, Liang \& Luo, 1997; Iwasaki et al., 2001; Jager et al., 2005; Lossdorfer, Gotz \& Jager, 2002; Saito et al., 1991a; Saito et al., 1991b; Yamaguchi \& Kasai, 2005; Yang et al., 2000). These cytokines induce recruitment of endothelial cells (monocytes) that differentiate into osteoclasts after binding of RANKL (receptor activator of nuclear factor kappa ligand), an osteoclast differentiation factor (Ogasawara et al., 2004). On the ERR side, an animal study and one clinical association study revealed that absence or a mutant 
gene (less transcribed) of IL1- $\beta$ are respectively linked to increase in orthodontic ERR (Al-Qawasmi et al., 2004; Al-Qawasmi et al., 2003a). A possible biological explanation to this fact is that the unavailability of this cytokine compromises neutrophil chemotaxis, fibroblast mitosis, and ultimately, the healing of the PDL. This compromises restoration of the original physiologic conditions that would keep repair and resorption balance. The presence of this cytokine is typically associated with catabolic activity: reduction of osteocalcin, TIMP (MMP inhibitor) and alkaline phosphatase and increase in proteases. Initial catabolic activity is necessary to remove hyalinized areas and restore the normal physiology of the PDL. Another interesting fact is that the absence of this cytokine impairs PGE2 and NO production (Hurwitz et al., 1997; Ralston \& Grabowski, 1996), which favors anabolic activity in the bone mechanotransduction pathway mentioned in the previous section. Nitric oxide (NO) increases root and bone resorption in orthodontics (Akin, Gurton \& Olmez, 2004; Leitao et al., 2005; Leitao et al., 2004), an effect opposite to that in orthopedic reactions. Currently, there is no proven explanation for these contradictions. There might be still unknown relationships in these mechanisms, such as dosage-dependent changes in molecular effects (like already proven dosage-dependent PTH and PGE2 effects).

Some research has also been performed to analyze the role of cytokine family factors involved in osteoclast differentiation in orthodontics. Linkage disequilibrium was shown between orthodontic ERR and a marker for the TNFRSF11A gene (Al-Qawasmi et al., 2003b), encoding RANK, a receptor essential for RANKL-mediated osteoclastogenesis. Although RANKL gene transfer has been shown to increase tooth movement (Kanzaki et al., 2006), RANKL levels have also been linked to severe 
orthodontic ERR (Yamaguchi et al., 2006). Another focus of research is OPG (osteoprotegerin), a decoy receptor that competes with RANKL to bind RANK, which when bound leads to suppression of osteoclast differentiation and activation, also inducing apoptosis. OPG gene transfer to the PDL was shown to inhibit tooth movement and osteoclast differentiation (Kanzaki et al., 2006; Kanzaki et al., 2004; Yamaguchi et al., 2006).

Although PGE1 seems to increase the rate of tooth movement and root resorption (Lee, 1990; Spielmann, Wieslander \& Hefti, 1989; Yamasaki, Shibasaki \& Fukuhara, 1983; Yamasaki et al., 1984; Zhang, 1992), some of the PGE2 effects in orthodontics are still controversial. In bone biology, lower dosages of PGE2 were shown to have anabolic effects, whereas higher dosages appear to disrupt this effect and cause bone resorptionthis effect appears to be linked to a consequential increase in IGF-1 levels (Baylink, Finkelman \& Mohan, 1993). In orthodontics, there seems to be a consensus on the fact that PGE2 injections increase the rate of tooth movement (Bao, Zhao \& Rao, 1995; Chao et al., 1988; Kale et al., 2004; Kokkinos et al., 1993; Leiker et al., 1995; Roberts, 2004; Seifi, Eslami \& Saffar, 2003). In contrast to orthopedic studies, which typically show anabolic effects of PGE2 (Norrdin, Jee \& High, 1990), studies have reported increase in bone resorption alone(Boekenoogen et al., 1996; Chao et al., 1988; Kale et al., 2004; Leiker et al., 1995; Seifi et al., 2003; Yamasaki, Shibata \& Fukuhara, 1982). For ERR, there are studies that report an increase (Boekenoogen et al., 1996; Leiker et al., 1995), but there are also studies that report no significant effect of this molecule (Brudvik \& Rygh, 1991). The controversies regarding bone formation, resorption and ERR may be related to differences in the methods of these studies, including dosage of PGE2 and 
previously discussed limitations of histological methods. In high dosages, PGE2 also causes orthopedic bone resorption.

Although the research on growth factors, rate of tooth movement and ERR is not as abundant as the previously mentioned molecule categories, results show that they may have an important role (Krishnan \& Davidovitch, 2006). TGF $\beta$ was shown to enhance osteoclast differentiation, also attracting monocytes and fibroblasts (Bonewald \& Mundy, 1990; Filvaroff et al., 1999; Hock, Canalis \& Centrella, 1990; Karst et al., 2004; Koseki et al., 2002; Kubota et al., 1990; Massey et al., 2001; Mundy, 1991; Pilkington, Sims \& Dixon, 2001; Sells Galvin et al., 1999; Yan et al., 2001). IGF-I and II stimulate cell proliferation and differentiation and may have a role in orthodontic tooth movement (Canalis et al., 1989; Mochizuki et al., 1992; Raisz et al., 1993a; Spencer et al., 1991; Thaller et al., 1993). Administration of recombinant VEGF (vascular endothelial growth factor) increases the rate of tooth movement and the number of osteoclasts, whereas the inhibition of this growth factor has the opposite effect (Kaku et al., 2001; Kohno et al., 2005).

In a comparison study, the active form of Vitamin D enhanced tooth movement as much as PGE2, but PGE2 promoted higher number of Howship’s lacunae in the compression side (Kale et al., 2004). However, Vitamin D increased the number of osteoblasts in the formation side. According to the author, a possible explanation is that this vitamin promotes a coupled resorption/formation enhancement.

\section{Damage-associated molecular pattern molecules/ Alarmins}

Molecules released by damaged or dying cells have been termed damageassociated molecular pattern molecules (DAMPs). They contribute to the induction of 
inflammation and interact with pattern recognition receptors. Disrupted or injured cells recruit innate inflammatory cells by releasing DAMPs. They are protein, or non protein (e.g. ATP, uric acid) molecules which are typically inside cells, but are released to the extracellular medium upon cell damage, or are sometimes released by cells. Many receptors for DAMPs and PAMPs (pathogen associated molecular patterns) are shared, indicating that similarities exist between pathogen-induced and noninfectious inflammatory responses. The repertoire of molecules released varies according to the type of injured tissue, and, therefore, the associated inflammation varies accordingly. DAMPs can serve to accentuate (e.g. HMGB1) or inhibit (e.g. adenoside) an inflammatory response (Rubartelli et al., 2007).

Alarmins can be defined as endogenous molecules with several characteristics: 1) rapidly released following nonprogrammed cel death, but not released by apoptotic cells; 2) immune system cells can be later induced to produce alarmins without dying; 3) they recruit and activate receptor-expressing cells of the innate immune system, such as dentritic cells, and also promote adaptive immunity responses and 4) can restore homeostasis by promoting reconstruction of the tissue (Bianchi, 2007). Clearly, the definitions of Alarmins and DAMPs overlap. Defensins, cathelicidin, eosinophil-derived neurotoxin (EDN), and high mobility group box protein 1 (HMGB1) can be considered typical alarmins.

Alarmins can bind to different receptors such as TLRs or IL1R, which are classical receptors leading to inflammatory and immune responses. RAGE also appears to be a receptor that plays a key role in alarmin function. Alarmins can play specific roles in the metabolism of mineralized tissue. Two major examples of alarmins have been shown 
to affect bone: extracellular ATP (binding to P2X7R) and HMGB1 (binding to RAGE), which will be discussed below.

\section{HMGB1 and Bone}

HMGB1 plays a significant role in endochondral but not intramembranous ossification. HMGB1 KO mice die shortly after birth but the embryos reveal that long bone development is significantly compromised, whereas the calvaria appears unaffected HMGB1 moves from the cytosol of hypertrophic chondrocytes at the growth plate to be released into the immediate environment. The released molecule acts as a chemoattractant for invading osteoclasts, osteoblasts and endothelial cells (Taniguchi et al., 2007).

This is consistent with a recent report showing differences between marrow and calvarial derived osteoblasts to recombinant HMGB1. The results suggest that HMGB1 acts as a potent bone resorption signal within the confines of the long bone marrow microenvironment but it may have a distinct role in flat bones (Yang et al., 2007).

A model has been recently proposed to explain control of bone resorption by HMGB1, embased by a close relationship between inflammation and bone loss associated with the RANK/RANKL/OPG mechanism. The hypothesis maintains that the osteocyte release of HMGB1 into the bone microenvironment triggers the expression and release of potent bone resorption agents such as RANKL, TNFa and IL-6 from BMSCs, osteoblasts and macrophages upon bindng to the RAGE, TLR2 and TLR4 receptors. This HMGB1 is chemotactic to the alerted osteoclasts. In addition, HMGB1 might also trigger the release of pro-inflammatory cytokines including HMGB1 itself acting as such. In normal bone remodeling, the release of modest amounts of HMGB1 brings the appropriate number of osteoclasts and osteoblasts to a microcrack in the osteocyte network caused by 
microdamage during normal turnover. In the pathological setting, an adverse inflammatory stimulus such as infection or arthritic erosion, the massive one time release of this alarmin would mobilize an excessive number of osteoclasts and disrupt the remodeling response (Bidwell et al., 2008).

\section{P2X7 receptor: a possible mediator of orthodontic mechanotransduction}

A P receptor was initially defined as a purine receptor, but research showed that some of these receptors also bind to pyrimidine nucleotides and nucleosides (North \& Barnard, 1997; Rassendren et al., 1997; Surprenant et al., 1996). P1 and P2 differ consistently in the molecular structure, but functionally their mechanisms are variable. The functional differentiation in these receptors is denoted by the letter (X or Y)- X means that the receptor is ionotropic (ligand-gated ion channel), whereas Y means that the receptor is metabotropic (G-protein or tyrosine kinase coupled receptors) (North \& Barnard, 1997; Rassendren et al., 1997; Surprenant et al., 1996). These receptors are expressed by a wide variety of cell types, including osteocytes, osteoblasts, fibroblasts and monocytes (Gartland et al., 2001; Naemsch, Dixon \& Sims, 2001; Solini et al., 1999). This receptor forms a signaling complex (Kim et al., 2001) and some of the known mechanisms affected by them are related to neuronal, immunological, oral and skeletal physiology (Collo et al., 1997; Turner et al., 1998).

$\mathrm{P} 2$ receptors typically can be activated by ATP release by the same (autocrine) or by other (paracrine) cells (North \& Barnard, 1997; Rassendren et al., 1997; Surprenant et al., 1996). It has been shown that P2 activation leads to increases in concentration of intracellular calcium and PGE2 release (Naemsch et al., 2001). ATP, which activates this pathway, is typically released by bone, endothelial and epithelial cells and fibroblasts 
under stress conditions, acting as a DAMP/alarmin (Bodin \& Burnstock, 1998;

Burnstock, 1999; Furuya, Sokabe \& Furuya, 2005; Grygorczyk \& Hanrahan, 1997; John \& Barakat, 2001; Katsuragi \& Migita, 2004; Katz, Boland \& Santillan, 2006; Kerkweg \& de Groot, 2005; Milner et al., 1992; Milner et al., 1990b; Ohata et al., 1997; Patel et al., 2005; Romanello et al., 2001; Sauer, Hescheler \& Wartenberg, 2000; Yamamoto et al., 2003).

The P2X7 receptor, encoded by the P2X7R gene, was shown to induce cytolytic pore formation, release of interleukin 1 beta in immune cells, cell membrane blebbing and necrosis/apoptosis. Interestingly, it also forms a complex with cell surface proteins with known role in mechanotransduction, such as alpha-actins and beta-integrins. These facts lead to the investigation on a possible role of this receptor in bone physiology.

True P2X7R KO mice were developed by construction of a targeting vector (Solle et al., 2001). A cDNA probe specific to the mouse $P 2 X 7 R$ gene was synthesized by reverse transcription-polymerase chain reaction using 2 primers, which were designed based on the published rat cDNA sequence of the P2X7R gene. Total RNA isolated from the J774 A.1 mouse monocyte/macrophage cell line was used as the template RNA. This polymerase chain reaction product was 401 base pairs long and was cloned and sequenced to verify that it corresponded to the mouse $P 2 X 7 R$ gene. The probe was used to screen a $129 / \mathrm{Sv}$ mouse genomic library and to isolate a single positive genomic clone. Sequence analysis of BamHI subcloned fragments confirmed that this clone corresponded to the mouse $P 2 X 7 R$ gene. A targeting vector was constructed that inserted the neomycin resistance gene from the pJNS2 plasmid directly after the Arg505 codon, deleting from Cys506 to Pro532, which is in the carboxyl-terminal domain of the $P 2 X 7 R$ gene product. 
Homologous recombination of the targeting vector with the endogenous $P 2 X 7 R$ gene disrupts the carboxyl-terminal coding region of the P2X7R gene. 129/Ola-derived E14Tg2a ES cells were grown, transformed, and screened using standard methods. Targeted ES cells and mice carrying the mutant allele were identified using a probe specific to a genomic region upstream of the targeted locus. Chimeric mice derived from targeted ES cells were mated with C57B/6 mice originally for 7 generations.

The KO mice showed reduced mineral content and periosteal circumference in the femur (Ke et al., 2003). They also show increased trabecular bone resorption and reduced periosteal bone formation. A recent study also showed, using $\mathrm{KO}$ mice, that the activation of this receptor affects the linear response to mechanical loading of bone (Li et al., 2005). The basic mechanism was shear stress=>ATP release=> P2X7 activation=> increased intracellular $[\mathrm{Ca} 2+]$ and release of PGE2. The osteogenic response was $73 \%$ less in male P2X7R KO mice and it is not associated with cell apoptosis.

Activation of the P2X7 receptor by ATP causes release of IL- $1 \beta$ by means of intracellular calcium signaling and cAMP (Chakfe et al., 2002; Ferrari et al., 2006; Grahames et al., 1999; Gudipaty et al., 2003). Increases in extracellular calcium after mechanical stimulation lead to further ATP release, which magnifies the process. In vitro experiments showed that P2X7R KO mice monocytes and macrophages were not able to post-process and release IL-1 $\beta$ extracellularly, and cell media had undetectable levels of this cytokine. The secretion of IL1 receptor antagonist is also dependent of signaling through this receptor (Wilson et al., 2004). In bone, this cytokine stimulates resorption. Absence of the cytokine would theoretically result in increased formation. The results from the P2X7 receptor study apparently show that the effect of this cytokine is probably 
comparably minor in bone mechanotransduction, because a decrease in the level of this cytokine in bone would imply increased formation in the KO mice. The exact opposite was shown by the studies involving the role of the P2X7R in bone.

These details may have important implications for orthodontics. Testing the role of this receptor in tooth movement could give us evidence of whether the previously mentioned mechanotransduction pathways play a major role in tooth movement, and confirm the role of this cytokine in root resorption. We hypothesize that the absence of this receptor will result in deficient bone formation and resulting low levels of IL-1 $1 \beta$ will result in decreased catabolic activity in the compression sides of the PDL. The net result would be a decreased rate of tooth movement. Because previous studies showed a link between IL-1 $\beta$ and root resorption, a similar finding in this study would provide evidence for another stage of the root resorption pathway: that the P2X7 activation mediates the mechanism. The summarized evidence suggests that the absence of the P2X7R could result in a compromise of signaling in transduction mechanisms in the PDL and bone.

From the genetics perspective, there are two polymorphisms that lead to a null allele of the P2X7 gene, one of them affecting 1-2\% of the Caucasian population, has already been reported (Gu et al., 2004; Skarratt et al., 2005). In addition, one polymorphism was proven to impair ATP-induced IL-1 $1 \beta$ release from human monocytes and one to affect normal trafficking of this receptor (Sluyter, Shemon \& Wiley, 2004; Wiley et al., 2003). If the role of the P2X7 receptor in tooth movement is shown in an experimental model controlling for multiple variables, it could provide a strong base for association studies of these polymorphisms with ERR, and rate of tooth movement. 


\section{Optimization of $\mu \mathrm{CT}$ Data Processing for Modelling of Dental Structures in Orthodontic Studies}

\section{Introduction}

Many studies have been published on the accuracy of $\mu \mathrm{CT}$ measurements the relationship of instrument settings to the rendering of tooth morphology and the results of bone morphometry (Kim, Paik \& Lee, 2005; Kohler et al., 2005; Olejniczak \& Grine, 2006; Thomsen et al., 2005; Waarsing, Day \& Weinans, 2004; Yeni et al., 2005).

Recently, several authors have extracted anatomical contours from $\mu \mathrm{CT}$ data to construct FE models (Cattaneo et al., 2005; Rahimi et al., 2005). The accuracy and reliability of these methods depend not only on the algorithm used for segmentation, but also on the quality of the acquired data. 'Resolution' is one measure of image quality. Resolution refers to smallest interval measurable by a scientific instrument. In tomography, it is typically given by the linear size of one side of the pixel (for instance, 8 micrometer resolution). Definition is a broader term, and refers to the distinctness of the reproduced image and its fidelity to the object. High resolution is necessary, but not sufficient, for good definition. That is, a blurred or noisy high resolution image lacks definition.

Obtaining good definition $\mu \mathrm{CT}$ radiographic images and reconstructions is not trivial. The objective of this project was to provide guidelines and procedures for optimizing $\mu \mathrm{CT}$ acquired data for dental applications and to demonstrate the high definition images and segmentations obtainable with commercially available software. The model of $\mu$ CT used was Skyscan ${ }^{\circledR} 1072$ (Skyscan ${ }^{\circledR}$, Aartselaar, Belgium). The specific application is the development of models for the biomechanical analysis of orthodontics associated root resorption. 
Some external root resorption is normal on human root surfaces where cementoclasts (cells very similar to osteoclasts) and osteoblasts function in a dynamic equilibrium. However, this balance may be perturbed by changes in the environment due to pathogens, trauma, orthodontic loading or occlusal interferences (Hartsfield JK Jr, 2004). The literature on root resorption is particularly ambiguous because of controversial and contradictory findings (Krishnan, 2005). It is therefore crucial to develop biomechanical models with adequate control of variables. Optimized $\mu \mathrm{CT}$ image processing is a critical component of that process.

\section{Materials and Methods}

\section{Biomechanical Models of Orthodontic Responses}

The first study evaluated mechanical damage on the root surface derived from fatigue loading. A hypothesis is that pre-existing irregularities on the root surface act as loci of stress concentrations that could initiate resorption. In the experiments, laboratory fatigue loading of dog teeth with a MTS Bionix ${ }^{\circledR} 858$ (Minneapolis, MN-USA) machine simulates situations such as a premature contact, or orthodontically induced occlusal interferences (Fig. 1). To detect surface differences in $\mu \mathrm{CT}$ images and to build representative FE models, good $\mu \mathrm{CT}$ output definition and sophisticated solid modelling are required.

The second model addressed the biological aspects of root resorption and tooth movement. In this model, transgenic and inbred strains of mice were used to quantify orthodontic responses. Within an inbred strain, all the mice have practically identical genetic backgrounds and they are homozygous for all loci. The comparison of mice from a particular inbred strain to a transgenic mouse (such as a gene knockout mouse derived 
from the same original inbred strain) allows us to compare differences in the anatomy and function of craniofacial structures that are attributable to that single genetic change. Through this comparison, differences in anatomical characteristics of dental structures are minimized to optimize control of mechanical variables along the root. In addition, comparison of orthodontic responses such as root resorption and velocity of tooth movement can be performed between different strains of mice (or transgenic mice) to evaluate associations between the response and other variables such as bone structure.

For the latter model, G\&H® Wire Company (Greenwood, IN- USA) helped to develop a special pseudoelastic NiTi spring that can deliver forces as low as $1 \mathrm{cN}$ with a load-deflection rate below $1 \mathrm{cN} / \mathrm{mm}$. A mouth prop was bent to allow placement and checking of the position and activation of the spring, thus minimizing activation error (Fig. 2). The force level was measured with a transducer with 1cN of accuracy (Orthomeasurements ${ }^{\circledR}$, Fairfield, CT- USA). This level of accuracy in the force system helps to assure that the animals will always have a very reproducible stimulus, so that changes in anatomy can be detected through imaging with decreased variability.

\section{$\mu C T$ Acquisition of Radiographs}

The basic $\mu \mathrm{CT}$ acquisition settings are amperage, voltage and exposure. Amperage refers to the intensity of the delivered $x$-ray beam. Voltage regulates the spectrum curve that is emitted by the polychromatic source. The higher the voltage, the greater are the proportions of x-rays with high energy. However, data from the manufacturer show that most of the radiation is within the $40-60 \mathrm{KeV}$ range, independent of the voltage setting. Exposure is the duration of the exposure. A high exposure helps to 
assure that the entire specimen is exposed to the full spectrum range. The best contrast was achieved with $85 \mathrm{KeV}$ and 9 seconds of exposure at $116 \mathrm{~mA}$.

After these parameters were set, the acquisition of good images depended on the minimization of artifacts and the use of small $\left(0.23^{\circ}\right)$ rotational steps when performing $180^{\circ}$ scans. $360^{\circ}$ scans generate more alignment discrepancies between the radiographic images, so they were not used, although they may be useful for other applications where there are high-density objects such as implants in the specimen. If the distance between the object and the central axis is large on the radiographic image (large magnification) it is necessary to use small rotation steps to prevent loss of information (gaps filled by interpolation) on the periphery of the object. The drawback is the increase in scanning time. In the 20-30x magnification range, maximum detail was achieved by using the minimum rotational step setting possible $\left(0.23^{\circ}\right)$ in a $180^{\circ}$ scan. Using this setting, the definition of the later reconstructions was similar to a microradiograph of a histological section. $0.45^{\circ}$ increments decreased the scan time by $50 \%$, but the sharpness of the image was not acceptable for our purposes. For both models, the optimum quality images were taken with 8-11 micrometers resolution. The size of a resorption cavity is typically larger than 10 micrometers (the size of a mature osteoclast). Although the system can achieve resolutions of up to 2 micrometers, at resolutions below 8 micrometers it becomes practically impossible to accurately represent specimens of our size, because of the increased number of magnification alignment artifacts.

The most common artifacts are due to noise, beam hardening and alignment. Noise is related to imperfections in camera detection, heterogeneous x-ray emission, cosmic events, and the presence of air/dust. There are two 'functional' types of noise. 
One type is 'static' noise due to camera imperfections or dust on its surface. Static noise can be effectively eliminated by using a flat field, an image that is subtracted from each radiograph. The other is 'dynamic’ or random noise, which results from cosmic events, heterogeneity in x-ray distribution, or floating dust. Flat field correction can incorrectly subtract random noise, which changes in each frame. Its minimization is discussed next.

\section{Control of Point Noise}

How to deal with dynamic noise? One standard tool is to average different frames (Frame Averaging). This means taking more than one radiograph for each angular position. Naturally, this causes an increase in scanning time. This procedure takes care of most of the lower intensity noise, such as floating dust, but the stronger cosmic events still remain. A software tool recommended by the manufacturer is the Median Filter. It substitutes the median greyscale value for all pixels within a determined region on the radiograph. This is very effective in removing noise due to cosmic events, but it also blurs the edges of objects within an image. For the dog tooth fatigue model, this entails the risk of a significant loss of information about cracks, imperfections, etc.

A more conservative approach to dealing with dynamic noise is Random Movement. During the scanning of a frame, both the camera and the object move in synchrony. This causes the noise to 'wash out', or become a line with a lower grey value that is inversely proportional to the amount of the Random Movement. For instance, Random Movement of 80 means that there will be an 80-line range of vertical movement during the scanning of the frames. Just like point noise, this line can generate ring artifacts in the reconstruction. However, when dealing with mineralized tissue, these artifacts can be eliminated with greyscale thresholding. When that does not work (for 
instance, with low magnifications), ring artifact correction must be used during reconstruction. Large random movement with high frame averaging is a conservative alternative to the median filter approach to remove noise (Fig. 3).

The ideal settings for both experimental models are 8 frame averages and random movement of 100 lines. We found that random movement of 100 removes almost completely the ring artefacts, but less than that tends to increase dramatically their number, compromising the thresholding and segmentation processes later. Figure 3 illustrates examples of noisy reconstruction sections obtained when random movement and median filter were off and frame averaging was low.

\section{Control of Beam Hardening}

Beam hardening occurs when the lower energy $\mathrm{x}$-rays are absorbed by the outside of the specimen, allowing only high energy radiation to reach the camera. This causes the interior parts of the object to look more radiolucent than they really are in comparison to the outside of the object. In the acquisition process, beam hardening can be controlled with aluminium filters. The aluminium filter skews the spectrum towards the high energy range, thus minimizing the beam hardening effect. We used a $0.5 \mathrm{~mm}$ aluminium filter in our mouse scans to eliminate beam hardening issues that could interfere with bone density measurements. For the dog model, it was not necessary to use the Aluminium filter because bone density measurements were not be taken. The presence of highdensity objects (such as implants) may also lead to streak artifacts and loss of information. The information loss can be controlled with a $360^{\circ}$ scan, at the expense of image sharpness, because with $360^{\circ}$ scans the possibilities for alignment mismatches increase. 


\section{Control of Alignment Artifacts}

One of the most important and unique of all settings during $\mu \mathrm{CT}$ image acquisition is the alignment. There are two issues related to alignment: 'wobbling' and magnification discrepancies. To avoid 'wobbling', the x-ray focus, the rotation axis of the specimen stage, and the camera center must be on the same line. Although mechanical calibration of the machine should take care of this, it is difficult to attain a sufficient level of precision. Imperfections in the rotational mechanism and in the straightness of the specimen holder itself may lead to deviant movements of the stage. The result is a lateral misalignment of the radiographs that appears in the reconstruction as wing artifacts wing-shaped extensions of the object (Fig. 4). The misalignment varies gradually with the wobbling of the holder, which explains the wing shape after convolution. This type of artifact can pose a significant problem during subsequent image processing.

The $\mu \mathrm{CT}$ control software has an algorithm for alignment correction. It consists of calculating the average misalignments at the top, centre and bottom of the rotational stage using the central vertical line of the screen as the reference. Appropriate compensations are then made in the positioning of the camera (at lower magnifications) or source (at higher magnifications). Software compensations are also made for misalignment between the top and bottom of the camera. The software also provides compensation for offsets associated with the lifting of the specimen stage, which naturally changes the alignment. To perform this procedure, a cylinder balanced on a specimen stage must be used. This is a partial solution to the problem, because there can be inconsistencies in the 'straightness' of different specimen holders and cylinders and in the way they are gripped by the chuck. As a practical issue, the small cylinder tends to topple off during the scan. To solve all 
these issues, we designed a precision-machined (by Mechanical Engineering, School of Engineering and Technology, IUPUI) tool with a removable alignment cylinder. Thus, the specimen can be scanned on the same holder that is used for alignment (Fig. 5).

Unfortunately, there are other alignment issues that are more difficult to control. Cone-beam geometry has a drawback: magnification differences in the images at each rotational position. Any object that significantly extends laterally from the long axis of the holder is subject of imperfections in the reconstruction derived from magnification differences in the radiographs (Fig. 6). The equation below establishes the relationship between the expected difference in magnification and the position of the object between the source and the camera.

$$
\frac{a}{b}=\frac{d}{c}(1)
$$

The equation (1) demonstrates that the lower the pixel size (higher resolution), the more problems can be expected from magnification differences (c) in the radiographic images. The point of the object facing the source at 0 will be in a different position on the camera at the $180^{\circ}$ rotation step. These magnification inconsistencies (as well as lateral misalignments) can be visualized directly on the screen of the NRecon ${ }^{\circledR}$ reconstruction software when the two radiographic images, $180^{\circ}$ apart, are superimposed. In summary, an increase in resolution does not necessarily imply an increase in the definition of the object for all applications when using cone beam geometry.

This problem is not eliminated by software; however, it can be minimized. The smaller the size of the object (i.e. the closer it is in size to the long axis of the specimen holder), the lower the differences in magnification between two images 180 degrees apart. Naturally, this also means that the volume of interest in a particular object should 
always be centralized on the specimen holder. The only method to minimize this problem is to find, by trial and error, the minimum pixel size that does not cause magnification alignment artifacts on the reconstruction image. For the dog scans, we use the minimum (14x) magnification. For the mice, good results were obtained in the 23-30x range. Despite of all these technical problems, $\mu \mathrm{CT}$ reconstruction has proven to be reasonably precise for human dental applications (Kim et al., 2005; Peters et al., 2000).

\section{Reconstruction}

The reconstruction software made available by Skyscan ${ }^{\circledR}$, NRecon ${ }^{\circledR}$, provides the cross-sections based on the radiographs. For our purpose, NRecon smoothing and correction for beam hardening were not used because they often cause loss of valuable information.

\section{Post-Acquisition Alignment Correction}

In the reconstruction step, post-alignment correction reduces magnification and lateral misalignment artifacts. It gives the user an opportunity to customize the reconstruction output with pixel shifting. If the machine is aligned, the shift value should be close to zero. The alignment tool allowed for the machine to be aligned specifically at the magnification position determined for the object, so optimal pixel shift values were typically between -1 and +1 pixel.

\section{Greyscale Thresholding}

Greyscale thresholding consisted of clearing (making white) soft tissue from the data and establishing the darkest pixel as black. This minimized the amount of information that had to be loaded into the segmentation software (Mimics ${ }^{\circledR}$ Materialise ${ }^{\circledR}$, Leuven, Belgium). To threshold accurately, we left some soft tissue 
reference on an unimportant area of the specimen. This allowed us to find the best range to establish our greyscale. All soft tissues were made white during the process, and the pixel with lowest value in the histogram determined the darkest value (black). The range obtained was then recorded for further reconstructions.

\section{Segmentation}

We used Mimics software for segmentation. The first step was to construct a 'mask' (coloured group of pixels) that included all mineralized tissue previously thresholded. The second step was to use a region growing algorithm to render the tooth with a colour code, thus establishing a new mask.

The region growing algorithm consists on adding voxels that have fairly homogeneous greyscale values adjacent to the seeding voxel (Myers, 1995). The voxels that are added are determined by a specified threshold, or standardized by the software. As new voxels are added, the algorithm will start to consider the greyscale of the growing region and the limits of other voxels in the region.

This algorithm is excellent for delineating the boundaries of the object's surface structure. It allows the isolation of the tooth from the mineralized tissue mask while preserving all the irregularities and discontinuities that are typically removed by other methods. The compromise in definition is determined only later, during smoothing of the STL (triangulated surface-stereolithography) file. To use this approach, it was critical to minimize the number of (mainly alignment) artifacts that 'ankylose' tooth to bone along the space of the periodontal ligament.

After the tooth was separated from the mineralized tissue mask, a Boolean operator was used to subtract the tooth mask from the mineralized tissue mask to obtain 
the bone mask. To segment the PDL, air and tooth pulp in the remaining 'white space' were separated. To do this, 'assistant' pixels were added to the mineralized tissue (bone and tooth) mask to close the root apex. Then, a cavity fill tool, which marks all white pixels inside a cavity, was used to produce a new 'air + PDL' mask. The air space above the bone level was then deleted from this mask with the multiple slice edit tool. Because we consider the PDL to be within the bone-tooth interface, manual deletion was necessary near the alveolar crest to precisely determine the limits of the PDL space. After this, all structures were segmented (Fig. 7).

\section{Bone Morphometry, 3D Rendering and Solid Modelling for FEA}

To characterize bone structure, we used CTAn ${ }^{\circ} / \mathrm{CTVol}{ }^{\circledR}$ software (Skyscan ${ }^{\circledR}$ ) to select a bone sphere in a standard location between the roots of the tooth and calculated its morphometric parameters (Table I). Bone mineral density was obtained after calibration of Hounsfield units with 2 hydroxyapatite phantoms (densities of $0.25 \mathrm{~g} / \mathrm{cm}^{3}$ and $0.75 \mathrm{~g} / \mathrm{cm}^{3}$ ) that matched the dimensions of our bone specimen. The elastic modulus of bone can be approximated using its mineral density (Fig. 8) (Krischak et al., 1999; Lang et al., 1988; Lotz, Gerhart \& Hayes, 1990; Wachter et al., 2001; Wachter et al., 2002).

The bone, PDL and tooth were then 3D rendered in Mimics ${ }^{\circledR}$ using gray value interpolation, providing maximum accuracy for visual inspection. In these renderings, all surface pixels were represented (Fig. 9). Then, they were exported as STL files. The STL files were further edited and converted to C1 NURBS surfaces, and then to CAD solid models (.igs extension B-rep surfaces) using Raindrop Geomagic Studio ${ }^{\circledR}$ and/or Rapidform ${ }^{\circledR}$ software (Fig. 10) (Sun, 2005). The solid models can be edited, meshed and 
joined using I-DEAS ${ }^{\circledR} 10$ software and then exported to ANSYS ${ }^{\circledR} 10$ for finite element analyses.

\section{Discussion}

In the dog model, this methodology allows accurate solid modelling of defects associated with changes in root surface caused by deformations of the root during fatigue loading.

In the mouse model, it allows specific analyses on the role of bone structure (morphometry), root morphology and immune reaction, as well as measurements of root dimensions pre- and post- treatment. This leads us to a better understanding of individual variability in humans, given that the mouse genome often has correspondence to human genes. Susceptibility to root resorption attributable to orthodontic force is a trait that has been analyzed in several experiments. However, the complexities of tooth movement biomechanics make research on this concept very challenging.

One important issue is the root resorption measurement method. Measuring by counting cavities per area in histological sections, for instance, is a common approach (Al-Qawasmi et al., 2004; Al-Qawasmi et al., 2006). In contrast to volumetric measurements, this method does not necessarily give a result of net root resorption, because it could also reflect a change in the turnover of cementum on the surface of the root. In addition, given that root morphology is an important factor in root resorption, variation in the area chosen for the measurement could lead to different results of susceptibility due to, for example, different positions of the centre of resistance in the two strains. For instance, in a simple analysis, if the strains are genetically identical for everything except a gene that determines distal root length, we could end up with a 
paradox: choosing the mesial root for analysis could tell us strain B is more susceptible, while choosing the distal root could tell us that strain A is more susceptible. Naturally, depending on the selected area, the overall results of the study can change because of a change in the stress patterns on each root, thus changing the 'susceptibility' of a given strain with respect to a given gene, thereby making this type of comparison meaningless.

Measuring root resorption by overall and local volume changes in locations of similar stimulus, or comparing mice with similar root morphology, are probably better options (Chan \& Darendeliler, 2005; Chan et al., 2004a; Chan et al., 2004b; Chan et al., 2005; Darendeliler et al., 2004).

\section{Conclusions}

This investigation showed that obtaining good output from the $\mu \mathrm{CT}$ required a thorough optimization of all settings specific for each research application. The described methodology allows for the analyses of specific morphological and physiological variables, which can produce meaningful conclusions on the role of mechanical and genetic variables on orthodontic responses. 


\section{The Three-dimensional Mechanical Environment of Orthodontic \\ Tooth Movement and Root Resorption}

\section{Introduction}

Three fundamental biological responses are attributed to orthodontic loads: bone formation, bone resorption, and iatrogenic external root resorption. The former two make orthodontics possible, while the latter is the foremost medico-legal issue for orthodontists (Brezniak \& Wasserstein, 2002a; Brezniak \& Wasserstein, 2002b).

In the general view of orthodontic tooth movement, "bone formation is associated with the tension side, and resorption with a pressure (compression) side” (Krishnan \& Davidovitch, 2006; Reitan, 1951; Schwartz, 1932). Other concepts link tooth movement to the flexion of the alveolar wall, and/or indirect resorption caused by compressive ischemia and periodontal ligament (PDL) necrosis (Baumrind, 1969; Epker \& Frost, 1965; Melsen, 2001). The tension/compression theory is ubiquitous in orthodontic research(Mitsui et al., 2005; Nishijima et al., 2006; Yamaguchi et al., 2004). Orthopedics proposes several mechanisms of mechanotransduction (Robling et al., 2006b) but applicability to orthodontics is unclear and there is no analogy to root resorption.

In a recent review, it was reported that uncertainties regarding orthodontic loads and the mechanical environment have led to issues in basic science and clinical research (Ren, Maltha \& Kuijpers-Jagtman, 2003). For instance, rodents in which standard clinical armamentaria are utilized to apply orthodontic loads are exposed to stresses in their alveolar structures that are orders of magnitude higher than would be applied in humans (Kawarizadeh et al., 2003a; Kawarizadeh et al., 2004). Root asymmetries, multiple roots, and uncontrolled and/or unavoidable loading offsets produce complex and unintuitive 
stress patterns due to simultaneous displacements and rotations in all three planes (Viecilli, Katona \& Roberts, 2007). Histological results can be puzzling (Verna et al., 1999) if the sectioning plane orientations are not determined according to specific stress (loading) patterns. This is sometimes neglected in clinical and basic science studies in which biological responses are linked to orthodontic loads and/or "stresses" that are improperly characterized (Iwasaki et al., 2006). Although there are some attempts for clarification, e.g., parametric analyses and partial descriptions, knowledge gaps abound at virtually all steps of the cascade shown in Figure 11. In recent publications (Chen et al., 2007; Viecilli, 2006), even commonly accepted notions about the clinical load systems generated by typical orthodontic mechanisms are still being questioned.

There is no consensus on the underlying mechanisms that link the orthodontic mechanical environment (stresses and/or strains) to biologic responses. Before such a relationship can be established, it is essential to thoroughly understand and control the mechanical environment, as it has been done in orthopedic research (Li et al., 2005). To do so even qualitatively, the applied orthodontic loads must be realistically delineated and the mechanical behavior of the involved materials must be reasonably approximated. Unfortunately, some of this required information is unavailable, inadequately described, or ignored. Thus, greater emphasis must be placed on the understanding of the mechanical environment that is associated with orthodontic responses.

Because of the intricacies of the dento-alveolar assembly (complex shapes consisting of 3 different materials: tooth, bone and PDL,) closed-form analytical calculations to characterize the mechanical environment are, for all intents and purposes, impossible. Useful results are obtainable with experimental and computer-based 
numerical models. By far the most powerful and popular example of the latter is FEA (Finite Element Analysis).

In this numerical study, we focused primarily on one aspect of the cascade (Fig. 11), the detailed elucidation of the mechanical environment within the dental structures that are subject to known orthodontic loads. Specifically, we use FEA to calculate the stresses and deformations produced during the distal translation or tipping of a stylized right maxillary human canine into a $1^{\text {st }}$ premolar extraction site. In addition to the typically considered principal stress magnitudes, we place emphasis on their directions. With this approach, we can examine two critical ambiguities in the orthodontic tension/compression concept. First, the specific tissue in which the pertinent tension/compression acts is sometimes not explicitly stated. That is, it is occasionally unclear if the mechanical environment under consideration is acting within bone, root or PDL. Second, there is a general lack of attention paid to the direction of the tension/compression. We hypothesize that (1) stresses in bone, PDL and root do not follow a simple pattern as assumed in the tension/compression theory of orthodontic tooth movement, (2) tension and compression can coexist with various combinations of magnitudes and directions in the dentoalveolar structures and (3) stress directions play an essential role in the description of the mechanical environment.

The first descriptive FEA studies of orthodontic tooth displacement and stress magnitudes were published by Tanne and Burstone more than 15 years ago (Tanne et al., 1989b). Since then, FEM studies have been published about parameters that may influence orthodontics associated stress magnitudes and their patterns. Most are technical studies of PDL mechanical properties, including its linear/non-linear (Cattaneo et al., 
2005), isotropic/anisotropic (Provatidis, 2000), and viscoelastic behaviors (Krstin et al., 2002; Natali et al., 2004), and the absence/presence of principal PDL fibers (Qian, Chen \& Katona, 2001). Studies have also looked at the role of bone heterogeneity (Cattaneo et al., 2005). For the purposes of this study, the PDL is modeled as a relatively simple linearly elastic isotropic material. The nuances of non-linearity of the PDL, which slightly affects the displacement pattern of the tooth, will be discussed in a separate paper.

Intricate objects such as the dento-alveolar complex should be modeled in 3-D. For instance, even loads initially applied in one dimension can lead to the appearance of stresses in three dimensions, depending on the supporting constraints. If the cube (Fig. 12, A) is uniformly compressed in the $\mathrm{y}$ direction, it is free to expand in the $\mathrm{x}$ and $\mathrm{z}$ directions (Poisson's effect) and stress will exist only in the y direction, although strain exists in all directions. If the cube's expansion is constrained in the $\mathrm{x}$ or $\mathrm{z}$ directions, compressive stresses will exist in all directions because the constraints will also load the body in response to Poisson's effect.

Typically, it is difficult to decipher the effects of the 6 simultaneously acting 3-D stress tensor components (3 shear and 3 normal stresses). Moreover, this type of stress characterization will depend on the coordinate system. It is, therefore, useful to mathematically transform the general stress tensor into a simpler equivalent state of stress in which the shear stresses disappear, and the three associated normal stresses (i.e., the principal stresses) assume their peak magnitudes. Conceptually, this is achieved by appropriately rotating the aforementioned imaginary cube within the body (Fig. 12, B). The obtained maximum, intermediate and minimum principal stresses at a point are 
referred to as the $1^{\text {st }}(\mathrm{S} 1,) 2^{\text {nd }}(\mathrm{S} 2)$ and $3^{\text {rd }}(\mathrm{S} 3)$ stresses, respectively, all perpendicular to each other. The principal stress numbers (S1, S2 and S3) are defined by convention solely on the basis of their algebraic magnitudes, not their directions. The magnitudes and directions of the principal stresses are invariant, thus they are an ideal way to present stress field results. The directions are perpendicular to each other, and can be indicated by the direction cosines of the normal vectors to the stress cube surfaces. As noted above, these are the critical (principal) directions that are generally overlooked or deemphasized in orthodontic stress analyses.

The state of stress at a point can also be characterized according to its effect (Chen \& Lui, 2005). For example, one component, the hydrostatic stress, changes the volume of an element without altering its shape. The other component, the deviatoric stress, distorts the element proportions without altering its overall volume. The former is analogous to fluid pressure on a solid body and its magnitude is the average of the three principal stresses. The hydrostatic stress tensor has equal stresses in all directions but no shear, thus it can be represented by a simple number (scalar.) The deviatoric stress tensor is obtained by subtracting the hydrostatic stress from the 3 principal stresses, thus, it cannot be represented by a scalar quantity. The von Mises stress/criterion is often cited in biological studies. However, it could be questionable to apply von Mises stress to PDL, bone and tooth because it is a criterion based on energy principles involving the yield of ductile materials such as metals (Beer, 2002).

Thus, given the various ways in which mechanical environments are characterized and reported, coupled with great anatomic variability, uncertainties about mechanical properties and loading, and ambiguities regarding the involved tissues and stress 
directions, it may be easy to see why the biological mechanisms of tooth movement and root resorption remain largely unexplained. Nonetheless, there have been attempts to correlate biological activity with changes in the mechanical environment as characterized by the 6 stress components (Kawarizadeh et al., 2004). 2D principal stresses (Katona et al., 1995) or hydrostatic stresses (Dorow \& Sander, 2005). Due to the previously mentioned limitations, the results are not conclusive. In the literature, the mechanical environment is typically depicted with color-coded stress magnitude gradients superimposed on the structure, or line graphs that show the stress and/or strain magnitudes along specific paths, but until recently (Cattaneo et al., 2005), without regard for their directions. Thus far, there is no published comprehensive description of the orthodontics-associated mechanical environment within the dento-alveolar complex that includes not only the principal stress magnitudes, but also their directions.

For our descriptive purposes, a cylindrical coordinate system nomenclature affords a relatively precise and unambiguous way to discuss the mechanical environment in the alveolus even if it is not entirely applicable to the tapered root/socket. In a cylindrical coordinate system, the three mutually perpendicular directions are longitudinal, radial, and circumferential (hoop). The longitudinal direction is in the apicogingival direction. The radial direction could be represented by the spokes of a wheel and the tangential (or circumferential) direction would be the tangent to the wheel. Thus, on the mesial and distal sides of the tooth, the circumferential direction is in the buccalpalatal direction and the radial direction corresponds to the mesio-distal direction. On the buccal and palatal sides of the tooth, the circumferential and radial directions are the mesio-distal and the buccal-palatal directions, respectively. As an example, it will be 
shown that with crown tipping toward the distal, the maximum principal stress in the distal PDL changes from a radial tension in the apical half, to a longitudinal compression in the gingival half. These types of direction changes, typically overlooked, may have profound biological implications.

\section{Materials and Methods}

The presented model simulated canine distalization by tipping or by translation into a premolar extraction site (Fig. 13, A) The anatomies of the canine root and socket and the two proximal roots and sockets were based on published average sizes fitted to regular elliptical paraboloid geometries (Marseiller, 1977). (The cross-sections of the roots/sockets are elliptical. The longitudinal shapes, viewed from the mesio-distal and the bucco-palatal directions, and in-between, are parabolic). The uniform PDL was modeled $0.2 \mathrm{~mm}$ thick. Bone geometry approximated the real shape of the buccal and palatal alveolar plates.

Using Solidworks 2006 (Dassault Systems S.A., Concord, MA-USA,) a CAD (computer-aided design) model of all structures was constructed. To reduce modeling intricacy, the $3.2 \mathrm{~mm}$ difference in the levels of the alveolar bone crest on the mesial/distal sides vs. the buccal/lingual sides, was uniformly leveled to the average of the two. Accordingly, the buccal crown length was reduced by $1.6 \mathrm{~mm}$ (i.e., half the 3.2 $\mathrm{mm}$ difference) from the $9.5 \mathrm{~mm}$ average to $7.9 \mathrm{~mm}$ to match the modification. The root length average of $17 \mathrm{~mm}$ was increased by $1.6 \mathrm{~mm}$, totaling $18.6 \mathrm{~mm}$. A stainless steel bracket, centered $4.5 \mathrm{~mm}$ from the cusp tip (21 $\mathrm{mm}$ from the root apex,) was simulated so that realistic orthodontic loads could be applied to the slot. The bracket width was $3 \mathrm{~mm}$. 
The assembly of bone, teeth and PDL, in IGES file format, was imported into ANSYS 10.0 University Research Version (ANSYS Inc., Canonsburg-PA, USA,) the FEA solver. For node sharing at the interfaces, the generated volumes were digitally glued with a tolerance of $0.003 \mathrm{~mm}$. Iterative meshing was performed with SOLID92 10noded tetrahedron elements which could maintain element quality and preserve the original CAD generated geometry. The final model (Fig. 13, B) consisted of 210,239 elements and 285,423 nodes. The origin of the FE model coordinate system was defined at the geometric center of the elliptical cross-section of the upper right canine root at the level of the alveolar crest (Fig. 13) Mesial (x), buccal (z) and apical (y) are the positive directions.

All materials were assumed to be homogeneous, isotropic and linearly elastic with Poisson's ratios equal to 0.3. The Young's moduli of bone (average of cortical and cancellous bone), PDL (consistent with the toe area of an experimentally determined bilinear PDL), tooth, and stainless steel bracket were 12,200 MPa, $0.05 \mathrm{MPa}, 20,000$ MPa, and 200,000 MPa, respectively (Cattaneo et al., 2005;Vollmer et al., 1999).

Orthodontic loads were applied to nodes on the bracket to simulate two realistic movements, pure distal translation ( $\mathrm{M}: \mathrm{F}=\sim-11.67 \mathrm{~mm}$, see below) and simple distal tipping (M:F = $0 \mathrm{~mm}$.) In both loading conditions, the applied distal force was ( $\mathrm{F}=)-1.2$ $\mathrm{N}$, along with a calculated anti-first order rotation moment, $4.55 \mathrm{~N}-\mathrm{mm}$. The boundary conditions, assigned as zero 3D displacements at the bottom and lateral walls of the bone, simulated natural anatomical constraints.

To obtain the appropriate M:F for pure distal translation (i.e., the distance from the bracket to the axis of resistance,) a negative couple about the $\mathrm{z}$ axis was applied to the 
bracket and combined with an ad hoc approach for refinement of the final value.

Basically, the displacements of 500 uniformly distributed points on a line connecting the root apex with the coordinate system origin, extrapolated from nodal displacements near that path, were determined using an ANSYS function. The coordinates of the two points closest to zero displacement were used to interpolate the approximate location of zero displacement. Its vertical distance to the bracket was the necessary M:F (-11.67 mm) for translation.

Post-processing of results focused on the nodal stresses and displacements next to material interfaces. As an example, for the PDL side of the PDL/root interface, only data from the interface-adjacent PDL elements were extrapolated to the interface nodes. Thus, results from different materials were never combined and stress discontinuities across interfaces were preserved. For PDL and root, results were considered in two representative planes, with emphasis on the DAM plane (defined by the distal of the alveolar crest, up to the apex, and down to the mesial of the alveolar crest) because it is the plane in which the canine moves. Less emphasis was given to the (perpendicular) BAP (buccal-apical-palatal) plane. For bone, the results are presented in a general 3-D fashion with vector plots and cervical graphs, because, unlike with root and PDL, the DAM plane does not align with the more palatally located areas of maximum bone stresses; an interesting confounding consequence of arch curvature.

\section{Results}

To aid in the visualization of principal stress directions, each principal stress' $\mathrm{x}$ (radial,) y (longitudinal) and z (tangential) components were calculated with their ANSYS-provided direction cosines. The stress direction components do not have 
individual physical meaning. They are presented separately to facilitate spatial visualization of the principal stress directions. The results are generally presented in the format of a direct buccal view of the tooth. In each graph, the side of the tooth (distal or mesial) is identified and the results are plotted as a function of vertical distance from the root apex $(0.0 \mathrm{~mm})$ down to the CEJ $(18.6 \mathrm{~mm})$ for the root, or from the apex of the socket $(0.0 \mathrm{~mm})$ down to the alveolar crest $(18.8 \mathrm{~mm})$ for the PDL/bone interface. (The $0.2 \mathrm{~mm}$ difference reflects the thickness of the apical PDL.) Unless otherwise noted, discussions pertain to the DAM plane.

\section{Displacements and deformations}

Figure 14, A shows the calculated total root displacements produced by the tipping (1.2 N distally directed force, and $\mathrm{M}=0$ ) and translating (1.2 N distally directed force with an 11.67 x $1.2=14.0 \mathrm{~N}$-mm counter-clockwise couple) loads. The results indicate that the CRes in the DAM-plane was located approximately $8.2 \mathrm{~mm}$, or $44.1 \%$ of root length, from the average bone level. Similarly, the tipping CRot was located at 10.4 $\mathrm{mm}, 55.9 \%$. Figures 14, B and $\mathbf{C}$ depict the corresponding tooth deformations. It was observed that loads on the canine also deformed the adjacent sockets and that peak bone stresses also occurred in those adjacent sockets (Fig. 15).

\section{Root stresses}

In the DAM plane, root stresses during translation followed a relatively simple pattern. On the distal, tension (S1) increased linearly from the apex to its peak at the CEJ (Fig. 16, A). Moreover, S1 was overlaid by its S1y component. This indicates tooth 
bending. Consequently, the stress was predominantly longitudinal, with a small $\mathrm{x}$ component perpendicular to the root. The situation on the mesial was the same except that the predominant stress (S3) was compressive (Fig. 16, B). S2 on both sides was small, but non-trivial. On the mesial and distal it was predominantly radial tension or compression, respectively, both in reaction to the PDL (Figs. 16, C and D).

With tipping, the peak stress magnitudes in the root were approximately the same as with translation, but the stress fields were more complex (Fig. 17). On both sides of the root, the peak stresses (compression on the distal, Fig. 17, A and tension on the mesial, Fig. 17, B) occurred at mid-root. Furthermore, on both sides, the dominant principal stresses (S3 on the distal, S1 on the mesial) had Sy as the main component and Sx as the minor component. Thus, as with translation, tooth bending caused the dominant stresses, although in tipping the root was bent in the opposite direction. S2, the mainly radial principal stress, followed the patterns of PDL S1 on the mesial and PDL S3 on the distal (Figs. 17, C and D).

\section{PDL stresses}

In general, the magnitudes of principal stresses S1 and S3 within the PDL were only slightly smaller at the PDL/bone interface than at the PDL/root interface and their directional patterns were very similar. Thus, only results for the former are presented.

In translation, all principal stresses in the distal PDL were compressive (Fig. 18, A-D) with peaks near the apex. By convention, S3 is defined as the algebraically smallest principal stress, so when all stresses are compressive, S3 (which is in the radial direction, see explanation below) has the largest absolute magnitude. S2 (which approximates a hoop, or circumferential, stress) and S1 (longitudinal stress) overlay each other with 
magnitudes approximately 50\% of S3. Figure 18, C shows the components of S3, the dominant principal stress on the distal side. The S3x component coincided with S3 itself, indicating that the largest principal stress was compressive, primarily in the radial (x) direction. S3 also had a small longitudinal stress component, S3y, near the apex. S1 (Fig. 18, A) and S2 (Fig. 18, B) were virtually identical in magnitude, but the former acted primarily in the axial (longitudinal) direction (S1y) while the later was a hoop stress (S2z.) On the mesial side, not shown, the situation was a near mirror image analogue, with all tensile stresses, including the small longitudinal component at the apex.

With tipping, the PDL stress distributions were more complex (Fig. 19, A-C,) and the peak stresses were about three times higher than those with translation. And as with translation, the mesial and distal sides were virtual mirror images, with S1 reversing roles with S3. With tipping, however, the PDL stresses were sufficiently different in the apical vs. the gingival halves to be considered separately. On the distal side, all principal stresses in the apical half of the PDL were tensile, with peaks near the apex. In the gingival half, all principal stresses became compressive, increasing linearly to their peak values at the alveolar crest. The dominant stress in the apical half was S1, a radial tensile stress, S1x (Fig. 19, A). The main stress in the gingival half was S3, a radial compressive stress, S3x (Fig. 19, C). At approximately 40\% of S1 values, S2 (Fig. 19, B) and S3 (Fig. 19, C) in the apical half were tangential (hoop, S2z) and longitudinal (S3y) tensile stresses, respectively. In the gingival half, they were tangential (hoop, S2z) and radial (S3x) compressive stresses, respectively. S1 also had a nontrivial longitudinal (S1y) compressive component near the apex, Figure 19, A. S3’s longitudinal tensile component (S3y) along the entire gingival half (Fig. 19, C) is also noteworthy. Except for these two 
components, as mentioned above, the stresses on the mesial (not shown) were mirror images of the distal.

At the apex, the magnitudes of all stresses were very small, and the stress directions tended to a pure shear component (S1 and S2 were generally opposite at $45^{\circ}$ to the $\mathrm{x}$ and $\mathrm{y}$ axes) in tipping and translation. The largest principal stresses were just gingival to the apex, but when the bone constraint to movement decreased, the stresses in the PDL became small.

In the PDL, the difference between the average principal stresses (hydrostatic stress) and the principal stress tensors was small, thus the deviatoric stress tensor is minor compared to the hydrostatic stress. The data also revealed that the overall stress magnitude varies significantly cervico-apically and between the MAD and BAP plane along the PDL/bone interface in translation (Fig. 18, D) and tipping. In the BAP plane, $\mathrm{S} 1$ and S3 were at $45^{\circ}$ to $\mathrm{x}, \mathrm{y}$ and $\mathrm{z}$, revealing that the stress state is pure shear.

\section{Bone stresses}

The state of stress in bone is complex, irregular, and at times, surprising. 3-D color-coded depictions of magnitudes and directions, and polar graphs (Figs. 20 and 21) provide an overall view of the stress patterns. With translation, the highest tensile stress within bone was in the circumferential direction in the premolar socket wall, adjacent to

the canine (Figs. 20, A-C and 11, A-C.) Similarly, the peak compressive bone stress was within the socket of the incisor, (Fig. 20, C) also in the circumferential direction (Figs.

\section{1, B, D).}

If analysis is confined to the canine socket, the highest magnitude stresses were circumferential. Two of the peak tension areas were located near the alveolar crest, one 
area just buccal, another just palatal, to the thin interproximal distal bone. The third local tensile peak was located at the mesial interproximal bone (Fig. 20, A). Interestingly, the highest compression on the distal side was flanked by the two above mentioned tensile areas on the distal, and the above mentioned tensile area on the mesial was flanked by compressive areas just to the buccal and palatal (Figs. 20, C and 21.) Thus, viewed from the occlusal (Fig. 21), in a relatively short distance close to the adjacent sockets, the predominant stress, a hoop stress, changed from tension-to-compression-to-tension on the distal side, and from compression-to-tension-to-compression on the mesial.

The peak stresses occurred at, or near, the crestal bone, and with tipping, their patterns are similar to those with translation, except that the magnitudes are $\sim 4$ times higher (Figs. 22 and 23). Away from the alveolar crest, the stress patterns changed. For instance, on the distal surface of the socket, the peak stress was S3, a compressive hoop stress at the thinnest bone septum in the region. S1 and S2 were predominantly a tensile longitudinal stress and a compressive radial stress, respectively. Moving $11 \mathrm{~mm}$ apically, the stresses gradually changed directions. S3 became a compressive radial stress (a reaction to direct PDL loads,) S2 a compressive hoop stress and S1 converged to zero (Fig. 21, A). Thus, there was a swap in the directions of S2 and S3 as the bone became thicker. The effects of translation on the mesial bone surface were almost symmetric to those on the distal surface - a difference existed due to the dissimilar socket shapes mesially and distally. The stress with the maximum absolute value was S1, a tensile hoop stress. S2 was a tensile radial stress and S3 a compressive longitudinal stress. Approximately $12 \mathrm{~mm}$ apically (6.6 $\mathrm{mm}$ from the apex,) S1 and S2 remained tensile, but they gradually swapped their directions as S3 converged to zero (Fig. 21, B). 
With tipping, the patterns of stresses and their magnitudes near the alveolar crest were the same as with translation. However, moving apically a difference developed in the pattern of stress directions: instead of one swap in directions between principal stresses, there are two. In the distal side, the first direction swap was completed $\sim 5 \mathrm{~mm}$ apically (13.6mm from apex,) where S3 became a compressive radial stress (the reaction to PDL loads becomes the highest stress as the bone becomes thicker,) S2 a tensile hoop stress and S1 a small longitudinal stress (Fig. 23, A). Later, at about 9 mm (9.6 mm from the apex,) there was another swap. S1 became the radial stress (where the PDL loads changed senses,) S2 a small tensile hoop stress and S3 tends to zero. The stress patterns in the mesial side during tipping are approximately the mirror image of the distal side, but S1 and S3 swap roles (Fig. 23, B).

Near the apex, S1 and S3 changed directions and tended to symmetric values and directions at $45^{\circ}$ to the $\mathrm{x}$ and $\mathrm{y}$ axes, characterizing pure shear. The stresses also tended to have large shear components as they deviated away from the MAD plane regions of the alveolar crest and approached the BAP regions.

\section{Discussion}

The calculated location of the axis of resistance was slightly different from those found in other experimental and FEA studies. (Nagerl et al., 1991; Provatidis, 1999; Vollmer et al., 1999) (In 3-D, CRes and CRot must be thought of as axes rather than points as in 2-D. When 3-D results are depicted in 2-D schematics, as in Figure 14, A, it must be recognized that the symbols that mark the center locations are, in actuality, axes that run in-out of the plane, generally not at $90^{\circ}$. In asymmetric roots and other situations, axes of resistance can be different in each plane, not intersecting at the "center of 
resistance.”) Possible reasons for this variability include: modeling of the root as a (symmetric) paraboloid of revolution or as a "real” tooth rather than an elliptic paraboloid; different root conicities; bucco-palatal vs. mesio-distal bone level as the reference; different bone insertion level. For average Caucasian population measurements, (Marseiller, 1977) the elliptic paraboloid appears to be the best morphological idealization of the canine root. Additional variation can be expected if the PDL is modeled as anisotropic or nonlinear, with or without PDL fibers. Thus, caution is advised against blind clinical implementation of these results.

An interesting observation is that the proximal sockets were deformed by the orthodontic loads on the canine, Figure 15. Of course, this phenomenon cannot be detected in typical single tooth FE models. The implication is that clinically observed orthodontic movements of proximal teeth may not be entirely due to the attributed action of transeptal fibers (Stubley, 1976), particularly with thin interproximal bone.

With tipping, the $1.2 \mathrm{~N}$ distal force on the bracket caused the tooth to bend in such a way that it cupped toward the distal. With translation, the $1.2 \mathrm{~N}$ force caused the same cupping, but tooth deformation due to the $14.0 \mathrm{~N}$-mm counter-clockwise moment (buccal view) on the bracket must be superimposed. That moment by itself would cause cupping of the tooth in the opposite direction with a magnitude that is sufficient to produce net root cupping toward the mesial when combined with the force. Thus, tipping and translation bent the root in opposite directions (Fig. 14, B). This was also shown in an earlier 2-D FE model (Katona, 1994).

Furthermore, these bending distortions generated longitudinal stresses, by far the largest stresses in the root. With distal translation, on the distal side, usually referred to as 
the "compression" side, the root was entirely in tension (Fig. 16, A). On the mesial (the “tension”) side, the root was in compression (Fig. 16, B). With tipping, the distal apical half is generally considered to be "tension” while the gingival half is "compression." Yet, the entire distal of the root was in compression (Fig. 17, A). On the mesial side, there was a similar disparity, also in the apical half (Fig. 17, B). These examples illustrate the importance of defining not only the specific structure, but also the specific location within that structure, in which a state of stress is being reported or discussed.

With distal translation, the distal PDL is correctly characterized as in "compression" because all stress components, expressed in terms of the principal stresses (S1, S2, and S3) or in terms of their directions (radial, hoop, and longitudinal) were indeed compressive (Fig. 18). However, that description does not convey the fact that the radial component is $\sim 2.5$ times larger than the other two, which, perhaps more importantly, are nontrivial (Fig. 18, D). Similarly, the mesial side is the "tension” side. In tipping, on the distal side, the radial stress is S3 in the cervical half of the PDL, whereas it is $\mathrm{S} 1$ in the apical half. This illustrates the importance of observing stress directions when trying to correlate the mechanical environment with biological responses.

It was found that the stress distribution pattern within bone is intricate. There were locations with quickly changing simultaneous tensile and compressive stresses in different directions. The stress field complexity can be attributed, in part, to two factors. First, because the line-of-action of the force intersects the socket slightly buccal to the thin interproximal bone, a complex asymmetric condition is created. Second, the bending deformations of the relatively thin interproximal septum of bone, particularly near the alveolar crest, had profound obfuscating effects on the local bone stresses. So, even with 
translation, the principal stresses swap directions at least once, and their magnitudes vary considerably. In tipping, this swap occurs twice. Moreover, the stress patterns can change dramatically depending on the alveolar bone shape and/or the presence of adjacent sockets, which could cause even more stress pattern variations (such as high longitudinal loads at the top of the crest in more realistic models). Thus, bone stresses appear to be sensitive to the modeling approach and to the clinical circumstances. In any case, as with the root, characterizing bone as the "tensile" or "compressive" side is inappropriate. Practically, the asymmetry of the high bone stresses complicates the appropriate selection of histological sections for the evaluation of bone response, assuming that bone stresses play a role in orthodontic mechanotransduction.

\section{Impact on Biological Responses}

The primary purpose of this chapter was the presentation of the details and nuances in the stress fields of orthodontically loaded alveolar structures. The possible implications for the mechanotransduction of bone formation and bone and root resorption are briefly discussed below.

In general, the PDL stresses in the DAM plane were either entirely compressive or entirely tensile in all directions. However, it was demonstrated that the magnitudes of the tensile (or compressive) stresses were not equal in all directions. The ratios between radial, circumferential, and longitudinal stress magnitudes were 2:1:1 for translation (Fig. 18) and 3:1:1 for tipping (Fig. 19). These observations suggest the possibility that PDL stress directions, not only stress magnitudes, could play roles in the stimulation of biological responses. It is plausible that, for example, the radial stress component, because it pushes the PDL against/into bone and root, may have a larger impact on 
biochemical signaling (Roberts, 2005; Roberts, 2004) or cell damage at the bone-PDL and root-PDL interfaces than the circumferential and longitudinal stress components. It could also be possible that PDL cells do not respond differentially to specific directions, but they do so to a change in volume (caused by hydrostatic stress). In that case, seeking correspondence with the average principal stress would be better than with a specific principal stress. Although the overall calculated tensile and compressive areas in the PDL are consistent with the known patterns of bone formation and resorption, respectively, and root resorption,(Chan \& Darendeliler, 2006; Reitan, 1951) biological experiments are needed to evaluate the potential influences of stress directions. An interesting observation based solely on the presented data is that the tooth translation associated PDL stress gradients (Fig. 18, A-C) were inconsistent with the requirement that the bone socket must resorb uniformly along the entire length of its distal surface for translatory root displacement to occur. Thus, under the hypotheses that perfect translatory movement is clinically possible and PDL compression mediates bone resorption, some sort of threshold or saturation phenomenon must be involved. On the other hand, control could be attributed to the relatively flat hoop and/or longitudinal stress components (Fig. 18, D). The same discussion does not necessarily apply to the bone formation on the mesial side because formation is limited by the resorption on the distal side. (Roberts, 2005; Roberts, 2004)

It is noteworthy that, with both loading conditions, the dominant root stresses are longitudinal and that they are perpendicular to the adjacent dominant radial PDL stresses. If root structure is taken into account, then tensile and compressive longitudinal stresses would tend to separate or crush the dentinal tubules, respectively, and affect the overlying 
cementum differentially, particularly if there are preexisting root surface defects. This may impact root resorption in areas where osteoclastic activity is already present, for instance, due to orthodontic loads or dysfunctional occlusion, that cause additional fatigue-mediated root degeneration. There are regions in which bone resorption takes place and the root is in tension (the distal side under translation (Fig. 16, A), and the apical mesial half with tipping (Fig. 17, B) or compression (gingival half of the distal side with tipping (Fig. 17, A). Thus, although the PDL stresses better fit root resorption patterns, the possibility exists that root resorption is linked to combinations of root and PDL stresses in which the former enhances root susceptibility to the cellular activity triggered by the latter.

With the hypothesis that there is bone response dependency on stress directions, bone stresses alone do not correlate with tooth displacement patterns. In tipping, for instance, there are two swaps in stress direction along the root axis. Based on previously published descriptions of tooth movement, the direction swap at approximately $5 \mathrm{~mm}$ apically does not seem to have any observable experimental or clinical impact. However, as proposed for root resorption, above, the presented data does not exclude the possibility of a combination of PDL and bone stress magnitudes and directions that produce loci of bone formation and resorption in some complex fashion.

Another finding was that stress magnitudes were dependent on the type of tooth movement, in agreement with previous classic work (Tanne et al., 1989b). The primary implication is that the concept of an optimal orthodontic force magnitude depends on the type of tooth movement. The mechanical stimulus caused by the same force magnitudes in translation and tipping can be very different. Thus, one should speak about optimal 
orthodontic loads (the combined forces and moments), and in specific dentoalveolar morphological conditions, rather than force magnitude alone, because ultimately, stresses are the likely mechanical stimuli. However, to apply this concept clinically, future work should focus on the specifics of how stress patterns are correlated to orthodontic responses.

Finally, perhaps the main biomechanical limitation of the present model is the simulation of only static loads. Although these loads appear to be the main determinants of tooth displacement, they are always superimposed on normal function. Unfortunately, complete mechanical information to construct dynamic FE models is still unavailable. However, it is essential to view all aspects of the biomechanics response in the grand scheme of dynamic functional loads, which produce constant subperiosteal bone modeling, sometimes far from the PDL/bone interface (Roberts, 2005; Roberts, 2004). So far, there has been only one attempt in the orthodontic literature to systematically define the role of a static load superimposed on function (Roberts et al., 1984).

\section{Conclusions}

The mechanical environment associated with orthodontic tooth movement and root resorption is more complex than is generally appreciated. Often, it is counterintuitive.

1. In the same region of root, PDL and bone, there can be predominantly compressive stresses in one structure, but tensile stresses in another. Thus, when 
referencing a region of compression or tension, the structure in which it occurs must be specified.

2. At a given point in any of the dento-alveolar structures, such as within bone, it is possible to have coexisting compression and tension in different directions.

3. Even if the state of stress is purely compressive or tensile in a specific region of a structure, such as the PDL, the stress magnitudes can be different in different directions, i.e., the stress is 3-D and not purely hydrostatic. Thus, it is inappropriate to refer to orthodontic stresses simply as "pressure" or to perform simplified 1-D calculations taking force and root surface area into account.

4. The principal stresses can swap their directions (and denominations) within a structure. A stress magnitude graph often displays sudden changes in behavior (as in Figure 19), which are typically a result of a change in direction. Consequently, previously described mechanical environments, based solely on stress magnitude plots, can be confusing, difficult to understand and/or to correlate with biological responses.

5. To avoid ambiguities, when a description of the orthodontic mechanical environment is given in terms of compression and tension, references to a principal stress must include not only the structure, but also the predominant direction of the stress in question.

6. The mechanical environment of the PDL is consistent with previously reported areas of root resorption and bone formation/resorption, and is, most likely, somehow, the main initiator of orthodontic mechanotransduction. Biological experiments in animal models are necessary to investigate the role of stress 
directions, as they can affect the direction in which biological reactions take place. Because complex loading and root morphology in animal models causes non-trivial directional stress fields, careful choice of histological section planes based on FE-determined stress directions is highly recommended. 


\section{Comparison of Dentoalveolar Morphology in P2X7KO and WT Mice for the Development of Biomechanical Orthodontic Models}

\section{Introduction}

The purpose of this study was to evaluate the role of the P2X7 receptor (P2X7R) in maxillary dental and trabecular bone morphology. P2X7R is an ATP-gated ionotropic channel, and a key mediator of inflammation and bone responses (North, 2002). It can be activated by extracellular ATP, a danger signal from cells under mechanical stress (Bodin \& Burnstock, 1998; Milner et al., 1992; Patel et al., 2005; Schneider et al., 2006). This causes the accumulation of intracellular calcium, and the release of chemical mediators, such as PGE2 and IL-1 $\alpha$ and $\beta$, that have utmost importance in inflammation and bone biology (Brough et al., 2003; Ferrari et al., 2006; Gudipaty et al., 2003; Li et al., 2005; Lister et al., 2007).

Proper function of P2X7R guarantees a proper acute phase response, helping to modulate the later chronic inflammatory reaction, the lack of which could lead to overwhelming macrophage infiltrate, even more apoptotic and necrotic cells, and generalized tissue damage (Lister et al., 2007).

At the bone level, P2X7R activation has been shown to be an important mechanism in mechanotransduction. For instance, femurs of $P 2 X 7 R \mathrm{KO}$ mice had morphometric characteristics compatible with a lower formation and an increased resorption activity, leading to the conclusion that this $\mathrm{KO}$ mouse has a bone disuse compatible phenotype (Ke et al., 2003). Recently, it has been shown that $P 2 X 7 R \mathrm{KO}$ mice tibia have reduced sensitivity to mechanical loading, especially in males (Li et al., 2005). 
It has been suggested that IL-1 might have a role in tooth development and eruption, because IL-1 receptors are expressed by ameloblasts during the secretory phase of amelogenesis, and odontoblasts during dentinogenesis (Wise, Lin \& Zhao, 1995; Xu et al., 1998). These characteristics make $P 2 X 7 R$ a particularly suited candidate gene to investigate a possible mechanical and genetic interaction in tooth and bone development. In addition, the importance of P2X7R has been extensively studied in fibroblasts and osteoblasts, which have fundamental roles in the initiation of tooth movement (Yee, 1979; Yee, Kimmel \& Jee, 1976). Orthodontic tooth movement involves two fundamental processes possibly mediated by P2X7R: the metabolism of an associated necrotic-type tissue (hyalinized periodontal ligament tissue) and bone modeling/remodeling (Reitan, 1994; Roberts, 2005; Roberts et al., 1981; Roberts, 2004; Rygh, 1974; Rygh, 1976). External root resorption, an undesirable side effect of tooth movement, has been associated with the presence of macrophages, possibly linking it to a chronic inflammatory response (Brudvik \& Rygh, 1993a; Brudvik \& Rygh, 1993b; Brudvik \& Rygh, 1994a; Brudvik \& Rygh, 1994b; Rygh, 1974; Rygh, 1976; Saito et al., 1991a). A strong association has been reported between IL-1 beta and root resorption (Al-Qawasmi et al., 2004; Al-Qawasmi et al., 2003a).

Orthodontic responses are dependent on mechanical stimuli (stresses), which, in turn, largely depend on the morphology of the dentoalveolar structures and the applied load vectors (Choy et al., 2000; Tanne et al., 1991). Any alteration in dentoalveolar morphology, due to the lack of the P2X7R for example, would directly affect the PDL (periodontal ligament) stress patterns, and therefore all PDL stress-linked biological phenomena. This could complicate the comparison of bone modeling and root resorption 
in different mouse strains. When these responses are analyzed in a specific region, they could be caused solely by mechanical environment changes, not by purely inflammatory effects, thus not characterizing a true physiological difference at the reactive inflammatory level.

Appropriate structural models are essential for FEM (Finite Element Method) calculations of the stresses and strains within dentoalveolar structures. Thus, this preliminary study compares the dentoalveolar morphologies of $P 2 X 7 R \mathrm{KO}$ (in the C57B/6 strain) and C57B/6 WT mice using microCT-based and histological methods. Specifically, a protocol for the evaluation of their maxillary first molars, the subject of a subsequent experimental study, and their surrounding trabecular bone, was developed. We hypothesized that there would be no gross differences in their dentoalveolar morphology. Failure to reject the hypothesis would justify a single structural model to represent both strains. On the other hand, two structural models, one specific to each strain, would be necessary if the hypothesis is rejected. Furthermore, the latter would require the development of a strategy to normalize biological responses according to the stresses in each strain of mouse.

There are no morphometric data on BMD (bone mineral density), the structure of maxillary trabecular bone, or tooth morphology for the C57B/6 WT and P2X7R KO mice. Additionally, it has been demonstrated that the interaction of genetic and nongenetic factors influence bone quantity and architecture differentially in different bones, to the extent that, even within the same bone, information from a specific site cannot be extrapolated to another (Judex et al., 2004). Thus, the necessity for specific morphometric 
analysis of mineralized tissue at the site of interest for the purpose of estimating its material properties is further justification for this study.

\section{Materials and Methods}

This animal study was approved by the Indiana University School of Dentistry IACUC. A total of 31 adult male mice (19 WT and $12 \mathrm{KO}$ ), sacrificed at 17 weeks and 3 days ( \pm 3 days) of age, were used in this study. The WT mice were purchased from Taconic Farms, Inc (Germantown, NY- USA). The KO mice were obtained from a colony maintained by the Orthopedic Biomechanics Laboratory at Indiana University School of Medicine (IUSM). After arrival, the animals were allowed at least 1 month of acclimation. They were euthanized by inhalation of carbon dioxide, and after decapitation, the heads were washed and put in NBF solution in the refrigerator for 1 day. Sections of the mice maxillas were extracted after dissection and fixed in the refrigerator for an additional day. Then, they were placed in 70\% ethanol solution and kept at ambient temperature. The maxillary sections were coated with paraffin and scanned using a Skyscan 1072 microCT (Skyscan, Kontich, Belgium). Reconstruction of the teeth in 2D transverse slices was performed using NRecon (Skyscan, Kontich, Belgium) software. All scans and reconstructions were standardized with optimized settings as described previously (Viecilli et al., 2007).

\section{Molar Morphology}

The reconstructed data were imported into Mimics 11.1 (Materialise, Aarstelar, Belgium). This software was used to segment and perform comparative measurements of the teeth, segmented as shown in Figure 7, and defined below. 
1. Crown length: distance between the midpoint of the mesial marginal ridge and distal marginal ridge.

2. Crown width: distance between the midpoints of the lines formed by union of the distal and mesial cusps, buccally and lingually.

3. Crown height: distance between the cemento-enamel junction and the occlusal plane of the maxilla.

4. Root length (measured on each root, mesial (M), disto-buccal (DB) and disto-lingual (DL)): distance between the apex and the bone insertion line, measured along the root canal.

5. Maximum root diameter (measured on each root): maximum root diameter below the furcation, measured perpendicular to the root canal.

6. Minimum root diameter (measured on each root): minimum root diameter below the furcation, measured perpendicular to the root canal.

7. Tooth volume: total volume of the mineralized tissue of the tooth.

\section{D Bone Morphometry}

CT Analyzer 4.1 (Skyscan, Kontich, Belgium) software was used to perform 3D bone morphometry. The bone used for analysis was sampled from a $0.5 \mathrm{~mm}$ diameter sphere (Fig. 8). The sphere was chosen so that it was equidistant to all roots, and located at the midplane between the mesial root apex and the furcation, parallel to the occlusal plane. To segment the bone from the rest of the tissue inside this sphere, a global gray value threshold was standardized for all specimens. Bone mineral density and Hounsfield Unit calibrations were performed using scans of water and hydroxyapatite rods (CIRS, 
Norfolk-VA, USA) of dimensions similar to the specimens and known mineral densities of $0.75 \mathrm{~g} / \mathrm{cm}^{3}$ and $0.25 \mathrm{~g} / \mathrm{cm}^{3}$.

\section{Histology}

Histology was conducted after the teeth were scanned to visually check for gross changes in tissue morphology such as disruptive developmental effects in cementum, dentin, enamel and PDL. The spatial position of the section plane was standardized. The maxillas were embedded in methyl-methacrylate, and ground to $20 \mu \mathrm{m}$ sections using a series of papers up to 1200 grit on an ExaKt system (ExaKt Technologies, Oklahoma City-OK-USA). The sections were polished down to 8-10 $\mu \mathrm{m}$ using papers of 2500 and 4000 grit. They were then deplasticized using acetone and xylene, and stained using a modified Masson’s trichrome stain protocol, which was optimized for MMA sections of teeth (Fig. 24).

\section{Finite Element Model}

After the results of the morphological comparison were known, the reconstructed sections of a specimen representative of average dimensions were imported into Amira (Visage Imaging, Dusseldorf, Germany) software. Bone, PDL and tooth were segmented into different bodies and smoothed to guarantee a mesh with high quality elements (free of ill-conditioned elements). The tissues were meshed with 4 noded tetrahedrons. The resulting model had strong anatomical fidelity, keeping the cortical bone and internal trabecular structure as distinct parts of the model (Fig. 25). Nodes of each material were shared at the interfaces. The model was imported into IDEAS 10, and saved in an IGES format to be imported into ANSYS 10, the finite element analysis software, where elements were converted into approximately 200K SOLID45 tetrahedrons. The material 
properties were established based on BMD of the representative specimen and previously determined rodent data (Kawarizadeh, Bourauel \& Jager, 2003b).

\section{Statistics}

Because of the reduced number of mice in the KO group, Mann-Whitney's nonparametric test was used to compare measurements between strains. The significance level was set at $5 \%$.

\section{Results}

The results for molar morphology comparison are shown in Table II. None of the measured dimensions revealed a statistical difference. The results for bone morphometry are depicted in Table III. Although none of the measurements revealed statistical significance at the 5\% level, there was a trend towards decreased trabecular number and increased trabecular separation in the KO group ( $\mathrm{p}=0.18$ and 0.13 respectively). The unexpected strongest trend was towards a higher bone BMD in the KO group, $0.91 \mathrm{~g} / \mathrm{cm}^{3}$ compared to $0.78 \mathrm{~g} / \mathrm{cm}^{3}$ in the WT $(\mathrm{p}=0.1)$.

Evaluation of the histological sections, performed with assistance from an experienced pathologist, did not reveal any typical noticeable difference in structural integrity of the tissues. Two KO mice demonstrated some mild level of enamel malformation, evidenced by brownish colored enamel spots similar to amelogenesis imperfecta that was detectable under the dissection microscope. Despite these findings, closer evaluation revealed no consistent differences in the mineral content or structure of teeth in the microCT or histology.

The lack of significant results in the morphological comparison leads to modeling of both the WT and KO mice with the same mechanical characteristics for orthodontic 
purposes. A single finite element model, representative of the approximate average morphological characteristics, was constructed to evaluate the stresses and strains in the PDL, and establish a rationale for histological cuts and biomechanical analyses in future studies (Fig. 26).

\section{Discussion}

The results demonstrated a trend towards increased SMI, Tr.Pf and Tr.Sp in KO mice, suggesting that $\mathrm{KO}$ mice have increased trabecular spacing, decreased connectivity and a weaker, rod-like trabecular structure. On the other hand, there was a trend toward increased BMD in the KO mice. Although these trends are not statistically significant, their combination suggests a possible compensatory mechanism for alveolar bone strength in the $P 2 X 7 R \mathrm{KO}$ mice: the tendency to poor trabecular structure is compensated by higher mineral content.

This is in partial contrast to previous studies that showed significant differences in bone morphometric parameters between the $P 2 X 7 R \mathrm{WT}$ and $\mathrm{KO}$ mice in other bone sites. Studies performed in the femur and tibia revealed a tendency toward lower BMD, higher catabolic, and lower anabolic bone activity in the KO mouse under unloaded and artificially loaded conditions (Ke et al., 2003; Li et al., 2005).

In this study, adult mice (17 weeks and 3 days of age) were used, whereas in the original femur and tibia morphology comparison study by Ke et al., littermates and young adult mice ( 2 weeks and 9 weeks were used). The mechanosensitivy study by Li et al. also used older mice (16 weeks of age), to prevent interference of growth-associated bone modeling in the data. Although it is possible that differences in the developmental age 
might have caused the contrast between this study and the ones mentioned above, other biological complexities are probably the best candidates to explain it, as described below.

The immediate cause for the disagreement in the long bone and alveolar bone phenotypes is a difference in genetic control of bone development. The alveolar bone is formed de novo through intramembranous osteogenesis during development, and the long bones are developed based on a cartilaginous scaffold (endochondral osteogenesis). The formation of bone in endochondral osteogenesis involves the hypoxia and apoptosis of hypertrophic chondrocytes (Hirao et al., 2006). The metabolism of apoptotic cells is at least partially controlled by the release of cytokines such as IL-1 $1 \beta$, which is dependent on P2X7R activation (Chen \& Brosnan, 2006). Moreover, IL-1 $\beta$ induces inhibition of type II collagen expression, controlled by the JNK and c-Jun/AP1 pathways (Hwang et al., 2005), and ultimately results in inhibition of Sox-9, which controls chondrocyte differentiation (Kronenberg, 2003). Since $P 2 X 7 R$ KO mice can't adequately process and release IL-1 $\beta$, it is natural that only mineralized tissues formed by endochondral ossification, such as long bones, could be affected by this deficiency. An analogous example of the importance of the ossification pattern in the genetic influences on bone phenotype has been reported in the literature. TNFR-1 KO mice, which lack a receptor that binds to TNF- $\alpha$, showed an altered phenotype in endochondral osteogenesis but not in intramembranous osteogenesis (Lukic et al., 2005).

From a biomechanical/environmental standpoint, the differences between maxilla vs. femur/tibia results could also be attributable to complexities associated with the mechanism of bone mechanotransduction. It has been shown that long bones are affected by the frequency and magnitude of strain, and the behavior of the surrounding tissues 
(Burr, Robling \& Turner, 2002; Robling, Castillo \& Turner, 2006a; Robling \& Turner, 2002). The outcome of the loading patterns, bone formation or resorption, is dependent on the resulting expression of the biochemical regulators of bone remodeling. Absence of the P2X7R resulted in decreased secretion of PGE2 and bone formation in long bone under specific loading conditions. The alveolar bone is typically subjected to masticatory loads, which most likely have a different frequency and magnitude if compared to long bones. Since the loading frequency and magnitudes in the maxillary bones are typically smaller, the resulting small difference in PGE2 production would most likely not produce a different phenotype. It is also possible that mechanical loading of the PDL, as a result of occlusal loads, leads to a different gene expression and activation profile than the one that results from the loading of long bones. Due to different gene arrays controlling bone formation at different sites, the strain thresholds for an osteogenic response vary with location (Hsieh et al., 2001).

Our results confirmed previous findings that bone characteristics are very site specific (Judex et al., 2004). Biomechanical models of mineralized tissue should be constructed based on local analysis rather than on extrapolation of site-specific data to the entire skeleton. 


\section{Orthodontic Mechanotransduction and the Role of the P2X7 Receptor}

\section{Introduction}

The sustained cellular activity associated with orthodontic tooth movement is well defined (Krishnan \& Davidovitch, 2006; Meikle, 2006), but the initial mechanotransduction of the orthodontic response is obscure. The purpose of current study is to describe the role of the P2X7 receptor in the transduction of mechanical loading on the bone adaptation and external root resorption (ERR) responses that are associated with orthodontic loads.

To experimentally determine individual roles of molecular and mechanical factors in orthodontic responses, both need to be simultaneously controlled. Data on periodontal ligament (PDL) reactions following orthodontic force is restricted primarily to rodents. Extrapolating those results to humans would require appropriately scaled orthodontic force magnitudes. This problem was recently discussed in a systematic review that searched for evidence of optimal orthodontic force magnitudes (Ren et al., 2003). Moreover, the complex shape of rodent teeth requires histological sectioning planes that take the 3D nature of stresses and strains into account. For instance, it is incorrect to assume that "compression” and "tension” areas in the PDL are always along the line of action of the force, because typically tooth displacements and rotations occur in all three planes of space in these models.

Stress analysis with finite element (FE) modeling is the state-of-the-art procedure for defining the zones of compression and tension in the PDL. Thus, FE modeling is a reliable method for determining the optimal orientation for histological sections. Because of biomechanical principles that are familiar to orthodontists, the ideal sectioning plane 
should approximate the stress distributions associated with single rooted teeth tipping in one plane.

There are also biological complications involved in controlling orthodontic experiments. Most studies are performed on animals with diverse genetics, resulting in highly variable biological responses, which are difficult to partition into biological and mechanical variability. Despite attempts to establish relationships between mechanical stimuli and orthodontic responses (Katona et al., 1995; Kawarizadeh et al., 2004) the specific relationships remain elusive, and controversies remain on the specific roles of mechanics and biology in individual variability.

A clinically relevant biomechanical mouse model of orthodontic treatment was developed to overcome these difficulties. Among other advantages, mice share 99\% of their genes with humans, thus they are very good animal models for studies of human biology. Mice from an inbred strain share $99.99 \%$ of their genetic material. By using a single strain, the role of genetic variation in the dispersion of data is practically eliminated. Because inbred mice have the same tooth and bone morphology, they can be modeled using a single FE model (Viecilli et al., Submitted in 2008). Using stress results from an FE model of a human tooth, it is possible to calculate the orthodontic loads that cause similar peak stress levels in mice. Furthermore, it is possible to localize histological planes in which the stress magnitudes and directions approximate those of the human compressive and tensile zones in the PDL of single rooted teeth exposed to rotation in one-plane or uncontrolled tipping.

Knock-out (KO) mice are invaluable for studying the role of individual genes in eliciting a biological response. KO mice differ from the inbred wild-type (WT) strain 
essentially by the deletion of a single (knocked-out) gene. In this study, the role of the P2X7 receptor gene (P2X7R) in the initiation of orthodontic tooth movement was studied. A previous study revealed that the mice used in this study (P2X7R KO and C57B/6 WT) have similar dentoalveolar morphology and can, therefore, be represented by the same FE model (Viecilli et al., Submitted in 2008).

The P2X7 receptor is an ATP-gated ionotropic channel and a key mediator of inflammation and bone adaptation responses (North, 2002). It can be activated (opened) after binding to extracellular ATP, which is a danger signal from cells under mechanical stress (Bodin \& Burnstock, 1998; Milner et al., 1990a; Schneider et al., 2006). Opening of the channel causes the accumulation of intracellular calcium and the release of inflammatory mediators such as PGE2, IL-1 $\alpha$ and IL-1 $\beta$, all fundamental in the control of bone physiology (Brough et al., 2003; Ferrari et al., 2006; Gudipaty et al., 2003; Li et al., 2005; Lister et al., 2007).

Recent research has shown that this receptor has a major role in the metabolism of apoptotic and necrotic tissue. After mechanical trauma, damaged cells release ATP that leads to the activation of P2X7 in macrophages and other cell types, which in turn release IL-1 cytokines (Qu et al., 2007). This is a basic general transduction mechanism of mechanical stimulus into a biological response. The released cytokines affect non-bone marrow derived cells, which, in turn, release chemoattractants for neutrophils and lymphocytes (Chen \& Brosnan, 2006). The neutrophils can act quickly to eliminate apoptotic cells and prevent further necrosis. P2X7R KO-derived macrophages do not release IL-1 in response to ATP, resulting on an attenuated acute inflammatory response (Labasi et al., 2002; Lister et al., 2007). Normal function of the P2X7R results in an 
optimal acute phase response that extends the life cycle of neutrophils (Barlow et al., 2006). An inefficient acute response can lead to an overwhelming chronic response: massive macrophage infiltration, abundant apoptotic and necrotic cells, and generalized tissue damage (Lister et al., 2007).

P2X7R activation has already been shown to be an important mechanism in long bone mechanotransduction: P2X7R mice tibia have reduced sensitivity to mechanical loading due to decreased secretion of PGE2 (Li et al., 2005). Thus, the P2X7R is an ideal candidate gene for mediating the orthodontics response, which involves the metabolism of an associated necrotic-type tissue (hyalinized periodontal ligament tissue) and bone modeling/remodeling (Reitan K, 1994; Roberts, 2005; Roberts et al., 1981; Roberts, 2004; Rygh, 1974; Rygh, 1976). These processes are mediated by the P2X7R in other physiological systems. Moreover, external root resorption (ERR), an undesirable side effect of tooth movement, has been associated with the presence of macrophages in several studies (Rygh, 1974; Rygh, 1976; Saito et al., 1991a). In addition, a strong association has been previously reported between IL-1 $\beta$ and ERR in animal and clinical studies (Al-Qawasmi et al., 2004; Al-Qawasmi et al., 2003a). P2X7R has also direct clinical relevance because there are many functional human polymorphisms in the P2X7R gene, which can induce different levels of affinity of these receptors to ATP. One of these polymorphisms, a null allele that occurs in $2 \%$ of the Caucasian population, is especially important because it results in reduced expression of the ion channel by $50 \%$ (Gu et al., 2004). It is hypothesized that variability in the expression of the P2X7R gene is an important factor in the individual variation to applied orthodontic loads. 


\section{Materials and Methods}

\section{Animals}

This animal study was approved by the Institutional Animal Care and Use Committee of the Indiana University School of Dentistry. Data for this study were obtained from 86 male mice (55 C57B/6 WT and 31 P2X7R KO). The 16 weeks old ( \pm 3 days) mice were distributed in 7 groups: WT controls (19), WT controls+bone labels (9), WT force (18), WT force+bone labels (9), KO controls (12), KO force (10), KO force+bone labels (9). The mice that received no bone labels were euthanized at 17 weeks and 3 days of age, and the labeled mice were sampled at 17 weeks and 4 days for bone formation analysis. The animals were randomized and the examiner was blinded with regard to KO/WT strain identification by a coded system until the analysis was complete. The WT mice were purchased from Taconic Farms, Inc (Germantown, NY- USA). The KO mice were obtained from a colony developed by the Orthopedic Biomechanics Laboratory at Indiana University School of Medicine (IUSM). After arrival, the animals were allowed at least 1 month of acclimation, to compensate for their different origin. They were euthanized by inhalation of carbon dioxide, and after decapitation, the heads were washed and prepared for histological and microCT analysis.

\section{MicroCT Scans and 3D Reconstructions}

The mice heads were put in cold neutral buffered formalin (NBF) solution and stored in a refrigerator for 24 hours. Sections of the mice maxillas were extracted after dissection and fixed in NBF in the refrigerator for an additional day. Then, they were placed in $70 \%$ ethanol solution and kept at ambient temperature. The maxillary sections were coated with paraffin to prevent dehydration and scanned in a Skyscan 1072 
microCT (Skyscan, Kontich, Belgium). Reconstruction of the maxilla in 2D transverse slices was performed using NRecon (Skyscan, Kontich, Belgium) software. All scans and reconstructions were standardized with optimized settings as described previously (Viecilli et al., 2007).

The reconstructed data were imported into Mimics software (Materialise, Aarstelar, Belgium), where the left molars were separated from PDL and bone for quantification of tooth volume and movement (Fig. 27, A and B). 3D tooth movement was also quantified by measuring the minimum distance between the crowns in the nonlabeled force groups.

\section{D Bone Morphometry}

CT Analyzer (Skyscan, Kontich, Belgium) software was used to perform 3D bone morphometry. The bone used for analysis was sampled from a $0.5 \mathrm{~mm}$ diameter sphere, chosen so that it was equidistant to all roots, and located at the midplane between the mesial root apex and the furcation, parallel to the occlusal plane (Fig. 27, C). To segment the bone from the rest of the tissue inside this spherical sample, a global gray value threshold was standardized for all specimens. Bone mineral density and Hounsfield Unit calibrations were performed using scans of water and hydroxyapatite rods (CIRS, Norfolk-VA, USA) of dimensions similar to the specimens, and known mineral densities of $0.75 \mathrm{~g} / \mathrm{cm}^{3}$ and $0.25 \mathrm{~g} / \mathrm{cm}^{3}$. Details of this methodology have been described previously (Viecilli et al., 2007).

\section{Finite Element Models}

The left maxillary molar of a mouse was used to construct the FE model (Viecilli et al., Submitted in 2008). The segmentation and meshing was performed with Amira 
(Visage Imaging, Dusseldorf, Germany) software. Bone, PDL and tooth were segmented and smoothed to guarantee a mesh free of self-intersecting units. The tissue surfaces were meshed with four-noded tetrahedrons. The resulting model had strong anatomical fidelity, keeping the characterized complex internal trabecular structure as part of the model (Fig. 27, C). Nodes of each material were shared at the interfaces. The model was imported into IDEAS 10, and saved in an IGES format to be imported into ANSYS 10, the finite element analysis software, where elements were converted into approximately 200,000 SOLID45 tetrahedrons. The material properties used were obtained from rodent tooth displacement experiments on autopsy specimens (Kawarizadeh et al., 2003b). The PDL was modeled as a nonlinear (bilinear) isotropic material. The model is shown in Figure 27, D.

For the human FE model, a maxillary segment containing a right upper canine was constructed using computer-aided design (CAD) software (Dassault Systems S.A., Concord-MA, USA). The CAD file was then imported into ANSYS, where it was meshed with 200,000 10-noded tetrahedrons for FE analysis. The material properties were also based on previously determined human canine displacement experiments, and the PDL was modeled as a nonlinear (bilinear) isotropic material (Vollmer et al., 1999). The modeling process is shown on Figure 28. A thorough stress analysis of this model has been described previously (Viecilli et al., 2008).

\section{Force Systems and Stress Analysis}

To determine a clinically translatable force magnitude for the mouse tooth, a simulation was conducted using the human FE model. A relatively high tipping force $(1.2 \mathrm{~N})$ was applied to the bracket to produce a simple distal crown tipping of the canine. 
(An anti-rotation first order couple was also applied.) The mean nodal stress levels in all regions of the PDL were computed as a range. Then, orthodontic force was applied iteratively in the mouse FEM until the calculated human canine mean principal stress overall range in the PDL (-35 to 25KPa) was approximated. With 0.03N ( 40 times smaller than the $1.2 \mathrm{~N}$ human force), the mean principal stress range in the PDL was -25 to 35KPa in the mouse, similar to the calculated human levels. The appropriate force magnitudes to compare human to mouse response could be well estimated with this method, despite the inherent morphological differences between the teeth. Because the mouse maxillary first molar has three roots, each one has a different stress magnitude range: generally, smaller roots experience higher magnitude stress ranges than larger roots.

A custom mouse closed coilspring made of very thin 0.003 '” superelastic NiTi wire, with a lumen of 0.019’, was designed and then produced by G\&H Wire Co (Greenwood, IN- USA). Spring measurements were performed using a $0.01 \mathrm{~N}$ resolution transducer (Orthomeasurements, Fairfield, CT- USA). A pilot study was conducted to obtain the average line of action of the force in six mouse specimens. The springs were cut to $3 \mathrm{~mm}$ of length, to obtain a $0.03 \mathrm{~N}$ force with $2 \mathrm{~mm}$ of activation, and then tied with a metallic ligature to the left maxillary molar and incisor. At the incisor, it was also secured with composite resin. The spring insertion procedure and the final average 3D line-of-action are shown on Figure 29. Note that the FE calculated maximum compression directions are not coincident with the line of action of the force, because the tooth displacement demonstrated differential components in all three planes of space (simultaneous 3D rotations and translations). It is ideal to use principal stresses in this 
type of analysis, because orthodontic responses are associated with compression and tension.

From an engineering standpoint, maximum compression and tension are determined in 3D by the calculation of the 3 principal stresses. Each one occurs in a specific direction, which aids in the determination of the histological sectioning plane. Principal stresses are always compressive or tensile, invariant, and have only three ortogonal components. The three principal stresses can be mathematically obtained from a general (three shear and three normal components) state of stress by rotating the coordinate system used to describe them to a specific position, so that all the stresses become normal (no shear). Both representations of the stress state are physically equivalent, i.e., the effect of application of three principal stress components in a body is the same of application of any mathematically equivalent stress matrix with six stress components.

\section{Histology}

After the teeth from the control and force WT/KO groups were scanned in the microCT instrument, histological sections were prepared to: 1 . quantify the incidence of root resorption, 2. assess the extent of hyalinized PDL tissue, and 3. determine the cellular types involved in its resorption 4. measure bone formation.

The choice of root and section plane for the ERR study was determined by identifying the direction and magnitude of the FE calculated maximum compressive stress in the mouse PDL. This was found in the PDL of the disto-buccal root, the smallest of the three roots. In the PDL of this root, the highest principal compressive stress is minimum near the apex and reaches its maximum value near the furcation. This 
maximum value is equivalent to the maximum stress obtained by high tipping force of $1.2 \mathrm{~N}$ on the PDL of the maxillary human canine model. The schematic in Figure $\mathbf{3 0}$ illustrates the rationale for the choice of direction of the cutting plane. A more detailed mechanical description of stress directions was described in a previous FE study (Viecilli et al., 2008).

The bone label groups were injected peritoneally after 2 days (calcein green, 30 $\mathrm{mg} / \mathrm{Kg}$ ) and 9 days (alizarin complexone, 50mg/Kg) of applied force. The disto-palatal root was chosen for the bone formation analysis because it demonstrated a relatively constant tipping pattern of displacement at the tension side, which was consistent with stress levels calculated for typical human canine movement. In the PDL of this root, the maximum tensile stress is equivalent to the maximum value obtained from a maxillary human canine tipping with $0.5 \mathrm{~N}$ of force. The cutting plane position for bone formation was determined according to the maximum tensile stress directions in this root. The cutting plane was parallel to the one used for root resorption, passing through the long axis of the disto-palatal root. The movement tendency predicted by the FEM in the bone formation and ERR histological sectioning planes is shown on Figure 31.

Thin mineralized sections were produced to preserve nuclear and cytoplasm shape and improve cell identification (non-labeled groups), and to avoid layered superimposition and allow preservation of the fluorescent bone labels (bone labeled groups). The maxillas were embedded in MMA (methyl-methacrylate), and slowly ground using a series of papers up to 1200 grit on an ExaKt system (ExaKt Technologies, Oklahoma City, OK- USA), to obtain a section as close as possible to the plane of histological analysis, traced on the acrylic surface as determined by FEM. The sections 
were polished down to 8-10um (nonbone-labeled groups for ERR analysis) and 20um (bone-labeled groups) using papers of 2500 and 4000 grit. The nonbone-labeled sections were then deplasticized using acetone and xylene and stained using a modified Masson’s trichrome stain protocol optimized for MMA sections of teeth.

Each disto-buccal root was observed under a microscope with 75x magnification and photographs were taken. A standardized grid was superimposed to each figure using ImageJ (public domain NIH image editing software). The compressive side of the PDL of the disto-buccal root was then divided into 3 equally spaced regions according to finite element analysis data: (1) high stress (near furcation), (2) medium stress, and (3) low stress (near apex). ERR was measured by counting squares adjacent to the root that contained resorption cavities. The grid was set up so that each region would have approximately 10 squares (square size $\sim 35 \mu \mathrm{m}$ ). The final resorption index was obtained by dividing this number by the total number of squares in each region, i.e., a score of 0.4 means 4 regions out of 10 had resorption cavities. Similarly, the hyalinization score was obtained by counting the total number of squares that contained necrotic tissue, and dividing by the total number of squares in the compressive side of the PDL. These methods are a higher resolution modification of a previously developed protocol (AlQawasmi et al., 2006; Lu et al., 1999). The identification of cell types was performed with the assistance of an experienced pathologist.

The PDL of the disto-palatal root was chosen for bone formation analysis. Bone interlabel distance was quantified by averaging the distances between the center of the calcein green and alizarin complexone labels at 3 different locations within different regions of the PDL. The regions were determined by orienting the long axis of the tooth 
vertically, tracing parallel lines at the average cervical bone level and the apical contour of bone, and then dividing the interval in 4 equal segments (Fig. 32). The three calculated nodal stresses (n1, n2 and n3) and the three measured bone interlabel distances (d1, d2 and d3) indicated in the figure were averaged to obtain Stress (mean nodal stress in each region) and BILD (mean bone interlabel distance), respectively. This was performed with ImageJ by calibrating 250 micrometer bars according to the pixel size of the photograph. All the data from KO and WT specimens, in each region, were averaged within each type. The values were recorded for statistical regression.

\section{Statistics}

Mann-Whitney’s non-parametric test was used to compare single measurements (tooth movement, hyalinization score, tooth volume and ERR score) between the two different mouse types. Kruskal-Wallis analysis of variance and Tamhane post-hoc tests were used to compare root resorption in different regions of the disto-buccal root within each mouse type. More conservative, non-parametric tests (and the inter-quartile range as a measurement of dispersion) were chosen because the assumption of a Gaussian distribution of data was questionable ( $<20$ in each group). In some cases the data clearly did not follow a Gaussian distribution (for instance, ERR control section scores were skewed toward 0). This justifies the use of medians as the measurement of central tendency.

Linear regression was conducted to assess the relationship of bone formation with stress after a natural logarithmic transformation. Analysis of Covariance (ANCOVA) was used to compare slopes of the bone formation vs. stress lines in KO and WT mice. The significance level for all analyses was set at 5\%. 
Reliability analysis was performed by repeat measurement of 10 randomly chosen specimens/data points for each method, after an interval of at least one week, and calculating the Pearson correlation. The reliability coefficients of the measurement methods of root volume, bone morphometry, bone interlabel distances and hyalinization/root resorption scores, were $97 \%, 94 \%, 92 \%$ and $89 \%$ respectively.

\section{Results}

\section{Hyalinization and ERR}

Ten days after the force was applied, the KO mice had $~ 8 \mathrm{x}$ more $(\mathrm{p}<0.001)$ hyalinized (necrotic PDL) tissue than the WT (Fig. 33). There was no difference in the incidence of ERR between the regions for all control animals across both strains. For the WT force group, ERR was $1.8 \mathrm{x}$ higher $(\mathrm{p}=0.002)$ and $3.7 \mathrm{x}$ higher $(\mathrm{p}<0.001)$ in the area of high stress compared to medium and low stress, respectively. A similar pattern was noted for the KO force group: respective scores were $4.7 \mathrm{x}(\mathrm{p}=0.001)$ and $3.5 \mathrm{x}$ ( $\mathrm{p}=0.002)$, (Fig. 34).

When comparing root resorption between KO and WT mice in different regions, the Kruskal-Wallis test determined that there was a difference, and that RR was elevated in the force groups. The post-hoc test revealed that the significant difference was in the region of the highest compressive stress, with $27 \%$ more $(\mathrm{p}<0.02)$ root resorption craters in the KO mice (Fig. 35). There were no significant differences between WT and KO controls in any of the regions, nor in regions 2, or 3 in treated mice. ERR was rarely observed in tension areas, where hyalinization did not occur. The few observed lesions were very shallow compared to the ones that occurred in compressive zones. 
To confirm that 2D root resorption data was consistent in 3D, tooth volume between strains in control and treated animals after the 10 day experimental period were compared. Figure 36 illustrates the $\sim 10 \mu \mathrm{m}$ scan resolution of the microCT scan, and how it is able to detect resorption lesions. The test revealed that there was no difference in tooth volume between control animals after the experimental period. However, tooth volume was $8 \%$ larger $(\mathrm{p}=0.002)$ in the treated WT compared to the treated $\mathrm{KO}$, which is consistent with the elevated ERR in the KO animals (Fig. 37).

Histological sections revealed gross differences between the KO and WT. Typically, a larger number of neutrophils, characterized by lobular shaped nuclei and small cytoplasm, were detected accompanying a mass of macrophages that accumulated at the border of the hyalinized zone in WT mice (Fig. 38), which was a high stress area prior to bone resorption. In many WT sections the small neutrophils, identified by their characteristic nuclear shape, were active enough to penetrate the narrow hyalinization zone to resorb necrotic tissue. The KO PDL space was characterized by cells of larger cytoplasmic area and large distance between regular shaped nuclei, suggesting the predominant cells were macrophages. The presence of neutrophils was rare in the KO. The macrophages did not penetrate the compressed hyalinized tissue, although they accumulated at its periphery and failed to remove the hyalinized tissue as effectively as the WT cells. Severe ERR and undermining bone resorption occurred adjacent to the cellular mass in the region where the initial compressive stress after loading was maximum. 


\section{Bone Response and Tooth Movement}

The within mouse type comparison of alveolar bone morphometric characteristics of control and treated animals shows that orthodontic force causes significant bone resorption within the alveolar process and at surfaces away from the PDL/bone interface (Table IV). This is a manifestation of the rapid acceleratory phenomenon (RAP). It is especially evidenced by statistically significant decreases in BMD and BV/TV, as well as an increase in BS/BV in WT and $\mathrm{KO}$ mice.

Disto-palatal socket interlabel bone distances were used to evaluate the relationship between bone formation in each mouse type and the associated FE-calculated PDL maximum tensile stress. Regions 5 - 8 were used for analysis because they displayed no evidence of bone formation (growth) in the controls (Fig. 39) and because their maximum tensile stress directions were exactly aligned with the plane of the histological section. A typical histological section with corresponding stress calculation is shown in Figure 32. A natural logarithmic transformation of stress values allowed linear regression with bone formation measurements. All coefficients were statistically different $(\mathrm{p}<0.006)$ from 0 , revealing that a logarithmic function was statistically adequate to represent the relationship between bone formation and PDL stress (Fig. 40). In contrast, no statistically significant difference ( $>0.2$ ) was found between bone formation in the two different mouse types.

A previous study revealed no differences in the bone morphometry of P2X7R KO and WT control mice (Viecilli et al., Submitted in 2008). However, with force application, our results indicate a significant difference in the Structure Model Index (SMI) of the two mouse types (Table V). The WT mice had a slightly higher SMI 
$(p<0.05)$ with a minor tendency toward a rod-like trabecular structure. The data suggest that WT mice had slightly higher resorption activity in trabecular bone distant from the PDL interface.

After 10 days of force application, no statistically significant differences ( $\mathrm{p}>0.9$ ) were observed in the amounts of tooth movement of each mouse type. Only non-labeled mice were used for this comparison to avoid possible interference of the bone labels in the data.

\section{Discussion}

The difference in the extent of hyalinized tissue after 10 days indicates that $\mathrm{KO}$ mice are less efficient at removing necrotic tissue. These data can be explained by the lack of ATP-driven stimulation of IL-1 secretion via the P2X7 receptor, leading to a poor initial neutrophil response. This finding is in concordance with previous literature on the role of this receptor in necrotic tissue metabolism. It is important to note that both $\mathrm{KO}$ and WT mice had some residual hyalinized tissue after 10 days of force application. This must be taken into consideration when interpreting the results of this study, especially the lack of a difference between $\mathrm{WT}$ and $\mathrm{KO}$ in the amount of tooth movement.

It was possible to extrapolate the mechanical threshold for clinical hyalinization from the coordinated histology and FEM data. Assuming that little necrotic tissue resorption took place in the KO mice (Fig. 33), and that hyalinization occurs equally in areas of maximum PDL compressive stress in both man and mouse, then FE analysis reveals that the minimum necessary compressive stress to produce hyalinization in the human or mouse PDL is $\sim 10 \mathrm{KPa}$. In the human upper canine, the minimum load magnitudes to cause this stress are approximately $0.4 \mathrm{~N}$ during simple tipping and $1.2 \mathrm{~N}$ 
(plus a moment) during translation. Figure $\mathbf{4 1}$ is an illustration of the compressive stresshyalinization relationship. These data are relatively consistent with values reported by Reitan (Reitan, 1970; Reitan, 1994) who observed hyalinization in upper incisors (smaller teeth), with tipping forces as low as $0.3 \mathrm{~N}$. This preliminary data indicates that this type of analysis is a promising strategy to establish scientific evidence on optimal orthodontic force magnitudes in future experiments where the time course of tooth movement will be studied with this model.

Histology showed that severe ERR occurred mainly in areas with dense accumulations of leukocytes adjacent to high compression zones. This suggests that, in the PDL, dense inflammatory cell accumulation leads to tissue destruction, as seen in general inflammatory processes in the body. In the WT mice, despite considerable resorption of the hyalinized tissue in areas of medium stress, little ERR was found. The statistically significant differences, up to $\sim 4 \mathrm{x}$, in the incidence of ERR in the higher stress regions in WT provides strong evidence that a localized elevation in PDL stress leads to a dramatic increase in ERR. This is an important finding because this is the first study to control genetics and the mechanical stress in the histological sectioning plane.

The difference in ERR between mouse types occurred at hyalinized areas where the stress was the highest subsequent to force application, and where most cell accumulation occured. Near the furcation region of the mouse tooth, there is an interface between a high compressive stress region at the lateral surface of the alveolar crest, and relatively constraint-free region immediately occlusal to the crest that viabilizes leukocyte accumulation. This constraint-free region probably facilitates diapedesis from blood vessels to the area of high compression, resulting in resorption of root, bone and 
hyalinized tissue. From a clinical perspective, this observation explains why ERR occurs primarily at the apex of single rooted teeth: the apex is an area relatively free of constraints, and adjacent to an area of high compressive stress (the lateral compressed zone near the apex).

Compared to the equilibrated macrophage and neutrophil infiltration for orthodontically stimulated WT animals, KO mice had a massive macrophage response. This suggests that the KO PDL reaction is analogous to an inefficient acute phase response associated with inadequate levels of P2X7 and IL-1 $\beta$. An inefficient acute phase response leads to an exacerbated macrophage infiltration, with slow resolution of necrotic hyalinization and and excessive tissue damage, characterized by root resorption. The association of macrophages with root resorption has been reported previously,(Brudvik \& Rygh, 1993a; Brudvik \& Rygh, 1993b; Brudvik \& Rygh, 1994a; Brudvik \& Rygh, 1994b; Roberts et al., 1981) but the role of neutrophils has been overlooked. A very interesting contrast in the orthodontic catabolic process in the $\mathrm{KO}$ is that more root resorption was observed, but not more bone resorption away from the PDL-bone interface. Lack of IL-1 $\beta$ contributes to bone resorption away from the PDL-bone interface, consistent with the role of this cytokine in bone physiology. However, it also contributes to more catabolic activity (tissue damage) in the periphery of the necrotic areas of the PDL, consistent with the role of P2X7 and IL-1 $\beta$ in necrotic tissue metabolism. Moreover, the decreased bone resorption away from the necrotic areas (undermining resorption) in the KO mouse, associated with a lack of IL-1 $\beta$, contributes to a vicious circle, leading to a denser accumulation of macrophages, and more ERR. The objective immunohistochemical quantification of PDL cell populations and molecular 
markers in orthodontically stimulated WT and $\mathrm{KO}$ mice is an important goal for future studies to further clarify this dichotomy in the catabolism of bone and root/hyalinized tissue.

Although numerous investigators have suggested associations between genetic factors and root resorption (Al-Qawasmi et al., 2004; Al-Qawasmi et al., 2003a; AlQawasmi et al., 2003b; Al-Qawasmi et al., 2006; Harris et al., 1997; Ngan et al., 2004) the mechanical environment was an inadequately controlled variable. The current study is the first to appropriately control the mechanical environment and select histological sectioning planes with FEM. Thus, it was proven that the influence of genetics on ERR can be independent of mechanical and anatomical factors. In clinical terms, individuals with the same root morphology, who are orthodontically treated with identical mechanics, may have different ERR responses as a direct consequence of their genetic differences.

The lack of statistical difference in ERR between KO and WT in areas of low ( $<10 \mathrm{KPa}$ ) PDL stress suggests that keeping forces at the lowest effective level could be a viable strategy for minimizing ERR in all patients, particularly those being treated with the new anti-inflammatory drugs that block the P2X7 receptor, or with polymorphisms that cause reduced P2X7 function. Although the concept of very light therapeutic loads is appealing, it may not be always practical because orthodontic loads are relatively light mechanics superimposed on heavy functional and postural loads, which may increase after tooth displacement due to a different occlusal contact configuration (Al-Qawasmi et al., 2003a; Roberts, 2005). In addition, commonly used appliances have very often statically indeterminate force systems. Keeping orthodontics under control to avoid 
excessive occlusal trauma, and using statically determinate force systems when possible, are at least as important as using low stress mechanics.

Bone morphometry revealed a slight tendency toward decreased resorption away from the PDL/bone interface in the KO mice. Bone metabolism in this area is not directly influenced by the necrotic tissue, thus, this effect is consistent with the absence of the catabolic effect of IL-1 in the bone modeling and remodeling response. An important follow-up to the current study would be to study the rate of sustained tooth movement in WT and KO animals. Cytokines may affect the initiation and sustained tooth movement responses differently. For instance, patients homozygous for allele 1 of IL-1 $\beta$ (diminished levels of IL- $\beta$ ) had an accentuated ERR response attributed to a slow initiation of tooth movement (Al-Qawasmi et al., 2003a). However, in a recent clinical study, patients homozygous for allele 1 of IL-1 $\beta$ demonstrated increased rates of sustained tooth movement compared to other patients (Iwasaki et al., 2006). This inconsistency may be biologically valid and related to timing of the study (the initial presence of necrotic tissue,) or it may reflect an uncontrolled mechanical environment in the clinical setting. Relative to the current scientific literature, the present data suggests a differential role for cytokines in the initiation and sustained tooth movement phases, which is possibly associated with the period of necrotic tissue clearance. The controversy about the role of cytokines in initiating and sustaining tooth movement could be resolved by varying the timing of force application, mechanical environment and sampling sites in the current mouse orthodontic model.

For fluorescent label bone formation analysis, the disto-palatal root was used because it had lower (below $14 \mathrm{KPa}$ ) PDL maximum tensile stress magnitudes. It is 
important to note that little or no bone formation was observed on the relatively high tension side of the disto-buccal root. Instead, bone cavities with very few active osteoclasts were observed (Fig. 42). This suggests that a resorption phase occurred before 10 days. The lack of initial bone formation in high PDL tensile stresses levels can be clinically related to the tooth mobility associated with high force appliances such as headgears. Of course, in such cases, bone formation can occur to restore the original PDL width after the mechanical stimulus is halted. Alternatively, a resorption/formation cycle, a result of a delayed RAP, could be maintained to achieve a sustained response. Future studies can examine the dynamics of this process by observing bone formation at different time points.

Interestingly, bone remodeling and apposition were observed on the buccal alveolar periosteum of all treated mice (Fig. 42), but not in control mice. Because a mechanical correlation with stress patterns could not be found in this region, it is proposed that this effect is associated with a gradient in PGE2 concentration from the PDL to the periosteum of the buccal bone. PGE2 has a concentration dependent biphasic effect on bone: in high concentrations, it has a catabolic effect, and in low concentrations an anabolic effect (Raisz, Pilbeam \& Fall, 1993b). This possibility is further reinforced by the observation that, before formation, significant bone resorption occurred in the cervical areas of the buccal bone, which correspond to the areas of higher PDL tensile stress, and consequently, initially higher PGE2 concentration that probably decreased after the steady state was reached. The nonlinear relationship between bone formation and the maximum tensile principal stress could be explained by this same mechanism: above $10 \mathrm{KPa}$, a significant decrease in bone formation was observed on the disto-palatal 
root, which largely explains the non-linearity of the graph. In many specimens, near the alveolar crest, where stresses were the highest, there were no labels, or the distance between them was greatly reduced.

The remnants of hyalinized tissue after 10 days probably affected the results of the bone formation and tooth movement comparisons between the mouse types. P2X7 receptor activation is a possible pathway for the release of PGE2; however, no statistically significant effects in bone formation and tooth movement were observed in this study. The limiting factors for bone formation and tooth movement are bone resorption and the persistence of hyalinized PDL tissue. Thus, the lack of demonstrated statistical differences in bone formation and tooth movement does not mean that significant effects would not become apparent with longer treatment times.

\section{Conclusions}

1) There is a direct relationship between PDL compressive stress magnitude and the incidence of ERR.

2) Severe ERR is associated with the presence of necrotic (hyalinized) PDL tissue, which occurs mainly due to high compressive stresses.

3) Lack of the P2X7R gene (KO) contributed to a slower removal of the hyalinized tissue.

4) The results of this study complement previous evidence, suggest that ERR and hyalinized tissue metabolism are mediated by the ATP-P2X7-IL-1 $\beta$ mechanotransduction pathway.

5) Absence of the P2X7R gene caused increased ERR in the mouse model. 
6) Bone resorptive activity is slightly diminished away from the PDL/bone interface in P2X7R KO mice.

7) There is a small interval of nonlinear relationship between PDL tensile stress magnitude and bone formation. High tensile stresses do not initially lead to bone formation, although they may promote a delayed RAP response.

8) Stress analysis (e.g., FEA) must be an integral component of experimental mechanotransduction studies to adequately control the mechanical environment. 


\section{SUMMARY}

The first part of this investigation showed that obtaining good output from the $\mu \mathrm{CT}$ required a thorough optimization of all settings specific for each research application. The described methodology allowed for the analyses of specific morphological and physiological variables, which can produce meaningful conclusions about the role of mechanical and genetic variables in orthodontic responses.

The second part of this investigation, which analyzed the mechanical environment of human orthodontics, revealed that in the same region of root, PDL and bone, there can be predominantly compressive stresses in one structure, but tensile stresses in another. Thus, when referencing a region of compression or tension, the structure in which it occurs must be specified. At a given point in any of the dento-alveolar structures, such as within bone, it is possible to have coexisting compression and tension in different directions. Even if the state of stress is purely compressive or tensile in a specific region of a structure, such as the PDL, the stress magnitudes can be different in different directions, i.e., the stress is 3-D and not purely hydrostatic. Thus, it is inappropriate to refer to orthodontic stresses simply as "pressure" or to perform simplified 1-D calculations taking force and root surface area into account. The principal stresses can swap their directions (and denominations) within a structure. A stress magnitude graph often displays sudden changes in behavior, which are typically a result of a change in direction. Consequently, previously described mechanical environments, based solely on stress magnitude plots, can be confusing, difficult to understand and/or to correlate with biological responses. To avoid ambiguities, when a description of the orthodontic 
mechanical environment is given in terms of compression and tension, references to a principal stress must include not only the structure, but also the predominant direction of the stress in question. The mechanical environment of the PDL is consistent with previously reported areas of root resorption and bone formation/resorption, and is, most likely, somehow, the main initiator of orthodontic mechanotransduction. Biological experiments in animal models are necessary to investigate the role of stress directions, as they can affect the direction in which biological reactions take place. Because complex loading and root morphology in animal models causes non-trivial directional stress fields, careful choice of histological section planes based on FE-determined stress directions is highly recommended, which was performed in the fourth part of this investigation.

The results of the third part of this investigation demonstrated a trend towards increased SMI, Tr.Pf and Tr.Sp in KO mice, suggesting that KO mice have increased trabecular spacing, decreased connectivity and a weaker, rod-like trabecular structure. On the other hand, there was a trend toward increased BMD in the KO mice. This study is in partial contrast to previous studies that showed significant differences in bone morphometric parameters between the $P 2 X 7 R \mathrm{WT}$ and $\mathrm{KO}$ mice in other bone sites. Studies performed in the femur and tibia revealed a tendency toward lower BMD, higher catabolic, and lower anabolic bone activity in the $\mathrm{KO}$ mouse under unloaded and artificially loaded conditions. Several problems might explain this difference. In this study, adult mice (17 weeks and 3 days of age) were used, whereas in the original femur and tibia morphology comparison study by Ke et al., littermates and young adult mice (2 weeks and 9 weeks were used). The mechanosensitivy study by Li et al. also used older mice (16 weeks of age), to prevent interference of growth-associated bone modeling in 
the data. Although it is possible that differences in the developmental age might have caused the contrast between this study and the ones mentioned above, other biological complexities are probably the best candidates to explain it, as described below.

The immediate cause for the disagreement in the long bone and alveolar bone phenotypes is a difference in genetic control of bone development. The alveolar bone is formed de novo through intramembranous osteogenesis during development, and the long bones are developed based on a cartilaginous scaffold (endochondral osteogenesis). Since $P 2 X 7 R$ KO mice can't adequately process and release IL- $1 \beta$, it is natural that only mineralized tissues formed by endochondral ossification, such as long bones, could be affected by this deficiency. From a biomechanical/environmental standpoint, the differences between maxilla vs. femur/tibia results could also be attributable to complexities associated with the mechanism of bone mechanotransduction. Long bones are affected by the frequency and magnitude of strain, and the behavior of the surrounding tissues. The outcome of the loading patterns, bone formation or resorption, is dependent on the resulting expression of the biochemical regulators of bone remodeling. The alveolar bone is typically subjected to masticatory loads, which most likely have a different frequency and magnitude if compared to long bones. Since the loading frequency and magnitudes in the maxillary bones are typically smaller, the resulting small difference in PGE2 production would most likely not produce a different phenotype. It is also possible that mechanical loading of the PDL, as a result of occlusal loads, leads to a different gene expression and activation profile than the one that results from the loading of long bones. Due to different gene arrays controlling bone formation at different sites, the strain thresholds for an osteogenic response vary with location. 
Our results confirmed previous findings that bone characteristics are very site specific. Biomechanical models of mineralized tissue should be constructed based on local analysis rather than on extrapolation of site-specific data to the entire skeleton. The mechanical environment associated with orthodontic tooth movement and root resorption is more complex than is generally appreciated. Often, it is counterintuitive.

The fourth part of this investigation provided several insights on mechanism of orthodontic mechanotransduction the role of the P2X7 receptor. There was a direct relationship between PDL compressive stress magnitude and the incidence of ERR. Severe ERR is associated with the presence of necrotic (hyalinized) PDL tissue, which occurs mainly due to high compressive stresses. Lack of the P2X7R gene (KO) contributed to a slower removal of the hyalinized tissue. Complementing previous evidence, this investigation suggested that ERR and hyalinized tissue metabolism are mediated by the ATP-P2X7- IL-1 $\beta$ mechanotransduction pathway. Absence of the P2X7R gene caused increased ERR in the mouse model. Bone resorptive activity is slightly diminished away from the PDL/bone interface in P2X7R KO mice. There is a small interval of nonlinear relationship between PDL tensile stress magnitude and bone formation. High tensile stresses do not initially lead to bone formation, although they may promote a delayed RAP response.

Stress analysis (e.g., FEA) must be an integral component of experimental mechanotransduction studies to adequately control the mechanical environment. 


\section{TABLES}

Table I- Bone morphometry data from the sphere taken from the C57B6 mouse. Standard abbreviations are used.

\begin{tabular}{|l|l|}
\hline BMD & $0.82 \mathrm{~g} / \mathrm{cm} 3$ \\
\hline BV/TV & $46.67 \%$ \\
\hline BS/BV & $43.70 / \mathrm{mm}$ \\
\hline Tb.N & $3.63 / \mathrm{mm}$ \\
\hline Tb.Th & $0.1286 \mathrm{~mm}$ \\
\hline Tb.S & $0.095 \mathrm{~mm}$ \\
\hline Tb.Pf & $14.492 / \mathrm{mm}$ \\
\hline SMI & 3.6 \\
\hline DA & 0.263 \\
\hline
\end{tabular}


Table II- Comparison of maxillary molar measurements in WT and KO mice.

\begin{tabular}{|c|c|c|c|c|c|}
\hline \multirow[b]{2}{*}{ Measurement } & \multicolumn{2}{|c|}{ WT } & \multicolumn{2}{|c|}{ KO } & \multirow[b]{2}{*}{ p value } \\
\hline & Median & Range & Median & Range & \\
\hline Tooth Volume $\left(\mathrm{mm}^{3}\right)$ & 1.07 & 0.11 & 1.06 & 0.16 & 0.11 \\
\hline Crown Length (mm) & 1.16 & 0.14 & 1.16 & 0.08 & 0.82 \\
\hline Crown Width (mm) & 0.90 & 0.12 & 0.90 & 0.11 & 0.31 \\
\hline Crown Height (mm) & 0.76 & 0.18 & 0.74 & 0.18 & 0.18 \\
\hline M Root Length (mm) & 1.13 & 0.14 & 1.12 & 0.11 & 0.12 \\
\hline M Root mn Diam (mm) & 0.37 & 0.19 & 0.38 & 0.08 & 0.13 \\
\hline M Root mx Diam (mm) & 0.70 & 0.14 & 0.70 & 0.09 & 0.8 \\
\hline DB Root Length (mm) & 0.82 & 0.13 & 0.83 & 0.16 & 0.97 \\
\hline DB Root mn Diam (mm) & 0.23 & 0.13 & 0.23 & 0.08 & 0.75 \\
\hline DB Root mx Diam (mm) & 0.67 & 0.28 & 0.67 & 0.17 & 0.67 \\
\hline DL Root Length (mm) & 0.81 & 0.18 & 0.80 & 0.12 & 0.21 \\
\hline DL Root mn Diam (mm) & 0.32 & 0.15 & 0.32 & 0.11 & 0.87 \\
\hline DL Root mx Diam (mm) & 0.47 & 0.13 & 0.49 & 0.16 & 0.39 \\
\hline
\end{tabular}


Table III- Comparison of bone 3D morphometric parameters. BMD= bone mineral density; $\mathrm{BV} / \mathrm{TV}=$ bone volume fraction; $\mathrm{BS} / \mathrm{BV}=$ bone surface-volume ratio; $\mathrm{Tr}$.Th= trabecular thickness; $\mathrm{Tr} . \mathrm{Sp}=$ trabecular separation; $\mathrm{Tr} . \mathrm{N}=$ trabecular number; $\mathrm{Tr} . \mathrm{Pf}=$ trabecular pattern factor; $\mathrm{SMI}=$ structure model index; $\mathrm{DA}=$ degree of anisotropy; $\mathrm{FD}=$ fractal dimension.

\begin{tabular}{|c|c|c|c|c|c|}
\hline & & $T$ & & $\mathbf{O}$ & \\
\hline Measurement & Median & Range & Median & Range & p value \\
\hline $\operatorname{BMD}\left(\mathrm{g} / \mathrm{cm}^{3}\right)$ & 0.78 & 0.15 & 0.91 & 0.20 & 0.10 \\
\hline BV/TV (\%) & 45 & 10 & 45 & 7 & 0.65 \\
\hline $\mathrm{BS} / \mathrm{BV}(/ \mathrm{mm})$ & 50 & 15 & 49 & 16 & 0.99 \\
\hline Tr.Th (mm) & 0.08 & 0.02 & 0.09 & 0.03 & 0.83 \\
\hline Tr.Sp (mm) & 0.13 & 0.02 & 0.15 & 0.05 & 0.13 \\
\hline Tr.N (/mm) & 5.6 & 1.1 & 5.1 & 1.4 & 0.18 \\
\hline Tr.Pf (/mm) & -3 & 10 & -1 & 14 & 0.10 \\
\hline SMI & 1.0 & 0.8 & 1.8 & 0.5 & 0.23 \\
\hline$\overline{\mathrm{DA}}$ & 2.2 & 1.3 & 2.1 & 1.1 & 0.29 \\
\hline FD & 2.24 & 0.07 & 2.12 & 0.10 & 0.28 \\
\hline
\end{tabular}


Table IV- Comparison of bone morphometry parameters of alveolar trabecular bone before and after application of orthodontic force in $\mathrm{WT}$ and $\mathrm{KO}$ mice. $\mathrm{BMD}=$ bone mineral density; $\mathrm{BV} / \mathrm{TV}=$ bone volume fraction; $\mathrm{BS} / \mathrm{BV}=$ bone surface-volume ratio; $\operatorname{Tr} . \mathrm{Th}=$ trabecular thickness; $\mathrm{Tr} . \mathrm{Sp}=$ trabecular separation; $\mathrm{Tr} . \mathrm{N}=$ trabecular number; Tr.Pf= trabecular pattern factor; $\mathrm{SMI}=$ structure model index; $\mathrm{DA}=$ degree of anisotropy; $\mathrm{FD}=$ fractal dimension

\begin{tabular}{|c|c|c|c|c|c|}
\hline \multirow[b]{2}{*}{ Measurement } & \multicolumn{2}{|c|}{ Control } & \multicolumn{2}{|c|}{ Force } & \multirow[b]{2}{*}{ p value } \\
\hline & Median & Range & Median & Range & \\
\hline $\begin{array}{cc}\operatorname{BMD}\left(\mathrm{g} / \mathrm{cm}^{3}\right) & \text { WT } \\
\text { KO } & \end{array}$ & $\begin{array}{l}0.78 \\
0.91\end{array}$ & $\begin{array}{l}0.15 \\
0.20\end{array}$ & $\begin{array}{l}0.54 \\
0.57\end{array}$ & $\begin{array}{l}0.14 \\
0.14\end{array}$ & $\begin{array}{l}<0.001 \\
<0.001\end{array}$ \\
\hline BV/TV (\%) ${ }_{\text {KO }}$ WT & $\begin{array}{l}45 \\
45\end{array}$ & \begin{tabular}{|l|}
10 \\
7
\end{tabular} & $\begin{array}{l}26 \\
25\end{array}$ & $\begin{array}{l}10 \\
12\end{array}$ & $\begin{array}{l}<0.001 \\
<0.01\end{array}$ \\
\hline $\begin{array}{rr}\mathrm{BS} / \mathrm{BV}(/ \mathrm{mm}) & \mathrm{WT} \\
\mathrm{KO} & \end{array}$ & $\begin{array}{l}50 \\
49 \\
\end{array}$ & $\begin{array}{l}15 \\
16 \\
\end{array}$ & $\begin{array}{l}64 \\
74\end{array}$ & $\begin{array}{l}20 \\
16\end{array}$ & $\begin{array}{l}0.001 \\
0.008\end{array}$ \\
\hline${ }_{\text {KO }}$ Tr.Th $(\mathrm{mm})$ WT & $\begin{array}{l}0.08 \\
0.09\end{array}$ & $\begin{array}{l}0.02 \\
0.03\end{array}$ & $\begin{array}{l}0.07 \\
0.06\end{array}$ & $\begin{array}{l}0.02 \\
0.01\end{array}$ & $\begin{array}{l}0.001 \\
0.01\end{array}$ \\
\hline $\operatorname{Tr.Sp}_{\text {KO }}$ WT & $\begin{array}{l}0.13 \\
0.15\end{array}$ & $\begin{array}{l}0.02 \\
0.05\end{array}$ & $\begin{array}{l}0.16 \\
0.16\end{array}$ & $\begin{array}{l}0.02 \\
0.09\end{array}$ & $\begin{array}{l}0.008 \\
0.87\end{array}$ \\
\hline $\operatorname{Tr.N}(/ \mathrm{mm}){ }_{\text {KO }}$ WT & $\begin{array}{l}5.6 \\
5.1\end{array}$ & \begin{tabular}{|l|}
1.1 \\
1.4
\end{tabular} & $\begin{array}{l}3.8 \\
4.1\end{array}$ & $\begin{array}{l}1.6 \\
1.2\end{array}$ & $\begin{array}{l}<0.001 \\
0.19\end{array}$ \\
\hline${ }_{\text {KO }}^{\operatorname{Tr} . P f(/ m m)}$ WT & $\begin{array}{l}-3 \\
-1\end{array}$ & \begin{tabular}{l|}
10 \\
14
\end{tabular} & $\begin{array}{l}14 \\
10\end{array}$ & $\begin{array}{l}12 \\
10\end{array}$ & $\begin{array}{l}<0.001 \\
0.17\end{array}$ \\
\hline $\begin{array}{l}\text { WT } \\
\text { KO }\end{array}$ & $\begin{array}{l}1.0 \\
1.8\end{array}$ & $\begin{array}{l}0.8 \\
0.5\end{array}$ & $\begin{array}{l}2.0 \\
1.8\end{array}$ & $\begin{array}{l}0.5 \\
0.4\end{array}$ & $\begin{array}{l}<0.001 \\
0.22\end{array}$ \\
\hline $\begin{array}{l}\text { WT } \\
\text { KO }\end{array}$ & $\begin{array}{l}2.2 \\
2.1\end{array}$ & \begin{tabular}{|l|}
1.3 \\
1.1
\end{tabular} & $\begin{array}{l}2.9 \\
2.1\end{array}$ & $\begin{array}{l}2.3 \\
1.1\end{array}$ & $\begin{array}{l}0.57 \\
0.29\end{array}$ \\
\hline $\begin{array}{l}\text { WT } \\
\text { KO }\end{array}$ & $\begin{array}{l}2.24 \\
2.12\end{array}$ & $\begin{array}{l}0.07 \\
0.10\end{array}$ & $\begin{array}{l}2.08 \\
2.11\end{array}$ & $\begin{array}{l}0.08 \\
0.11\end{array}$ & $\begin{array}{l}<0.001 \\
0.11\end{array}$ \\
\hline
\end{tabular}


Table V-Comparison of alveolar trabecular bone morphometry after force application in $\mathrm{WT}$ and $\mathrm{KO}$ mice. $\mathrm{BMD}=$ bone mineral density; $\mathrm{BV} / \mathrm{TV}=$ bone volume fraction; $\mathrm{BS} / \mathrm{BV}=$ bone surface-volume ratio; Tr.Th= trabecular thickness; Tr.Sp= trabecular separation; Tr.N= trabecular number; Tr.Pf= trabecular pattern factor; $\mathrm{SMI}=$ structure model index; $\mathrm{DA}=$ degree of anisotropy; $\mathrm{FD}=$ fractal dimension.

\begin{tabular}{|c|c|c|c|c|c|}
\hline & \multicolumn{2}{|c|}{ WT } & \multicolumn{2}{|c|}{ KO } & \\
\hline Measurement & Median & Range & Median & Range & $p$ value \\
\hline $\operatorname{BMD}\left(\mathrm{g} / \mathrm{cm}^{3}\right)$ & 0.54 & 0.14 & 0.57 & 0.14 & 0.58 \\
\hline BV/TV (\%) & 26 & 10 & 25 & 12 & 0.61 \\
\hline BS/BV (/mm) & 64 & 20 & 74 & 16 & 0.27 \\
\hline Tr.Th (mm) & 0.07 & 0.02 & 0.06 & 0.01 & 0.31 \\
\hline Tr.Sp (mm) & 0.16 & 0.02 & 0.16 & 0.09 & 0.94 \\
\hline Tr.N (/mm) & 3.8 & 1.6 & 4.1 & 1.2 & 0.19 \\
\hline Tr.Pf (/mm) & 14 & 12 & 10 & 10 & 0.14 \\
\hline SMI & 2.0 & 0.5 & 1.8 & 0.4 & $<0.05$ \\
\hline DA & 2.9 & 2.3 & 2.1 & 1.1 & 0.41 \\
\hline FD & 2.08 & 0.08 & 2.11 & 0.11 & 0.23 \\
\hline
\end{tabular}




\section{FIGURES}

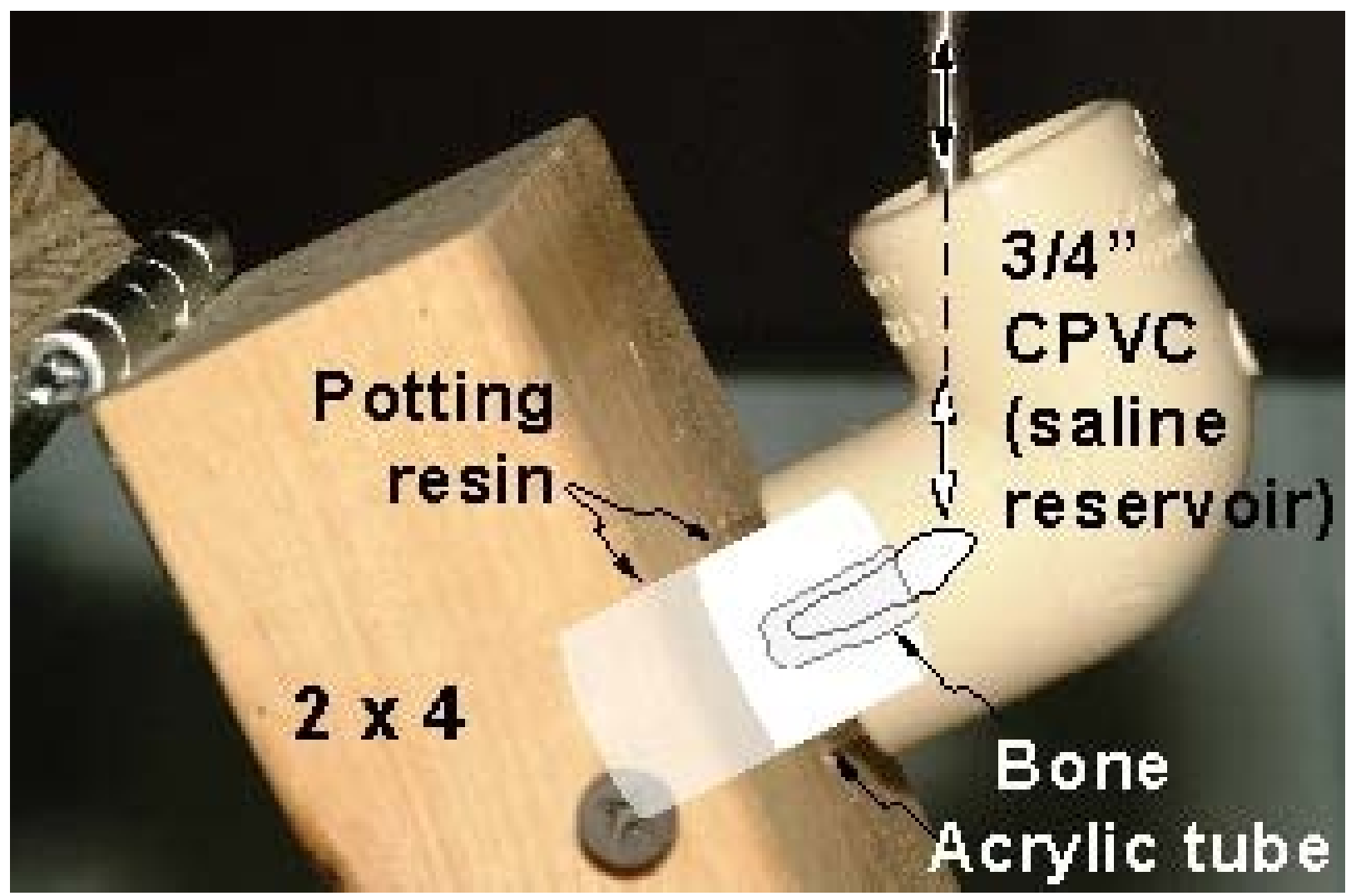

Figure 1. Fatigue experiment diagram: the apparatus for loading of the dentoalveolar structures of a mandibular lower incisor is illustrated. 


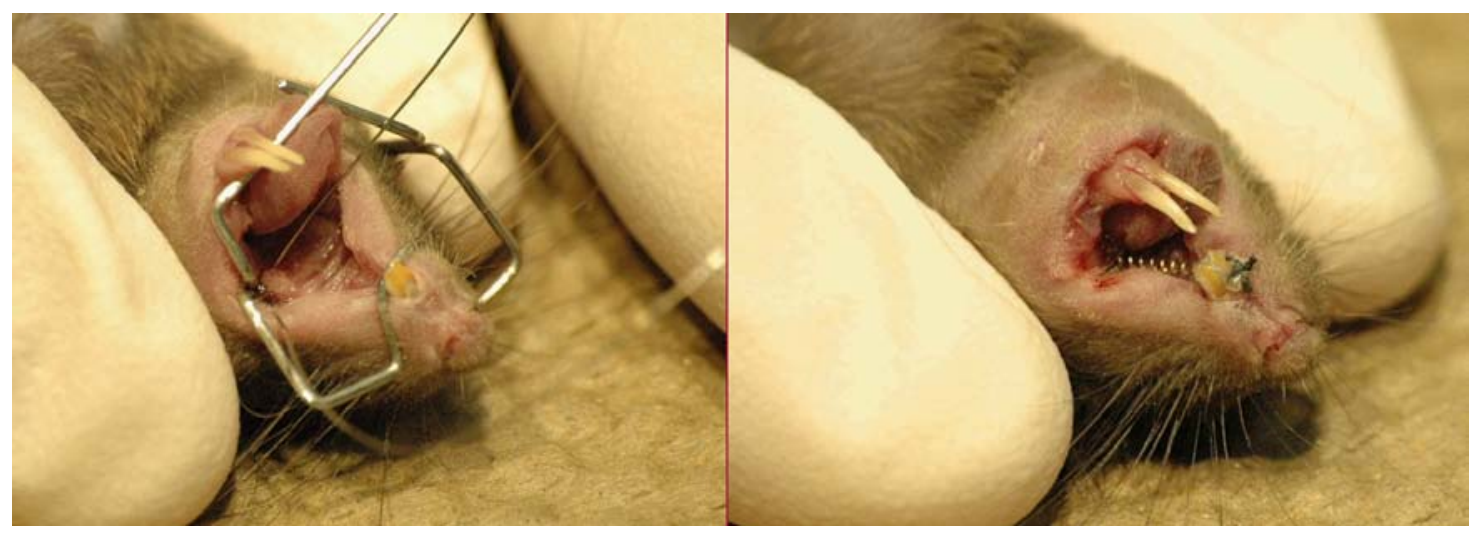

Figure 2. The newly developed NiTi coilspring and its insertion procedure. 

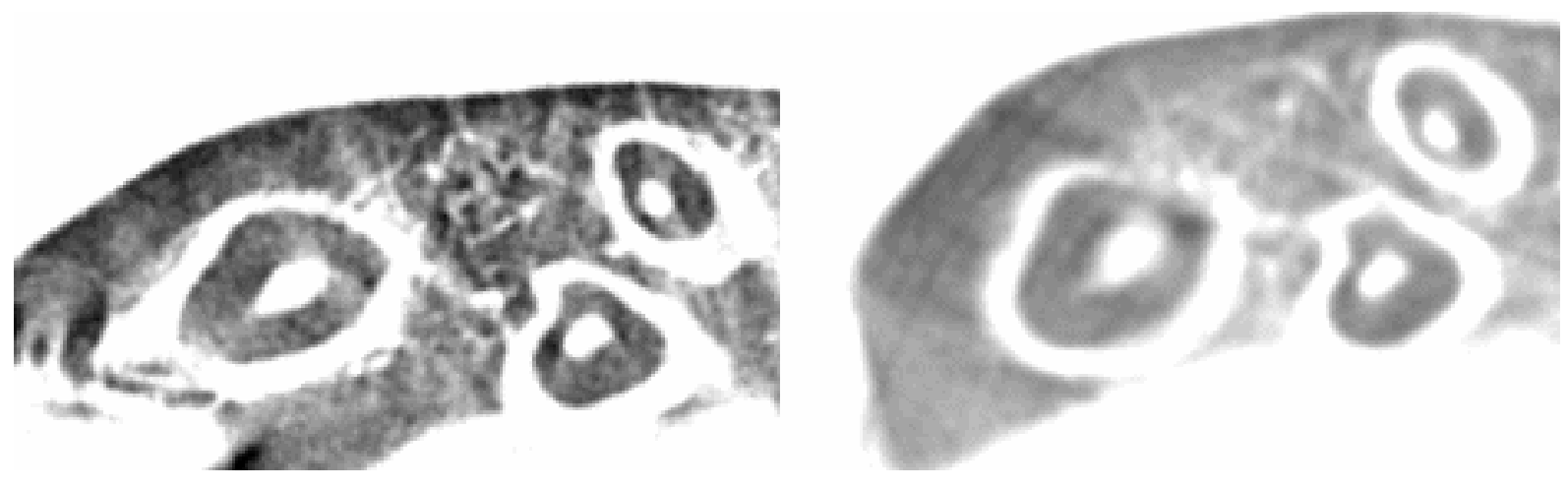

Figure 3. Sections scanned without (left) and with (right) median filter. Note the sharpness and detail of the left image in which random movement of 100 lines and averaging of 8 frames were used to remove noise instead of the Median Filter. 

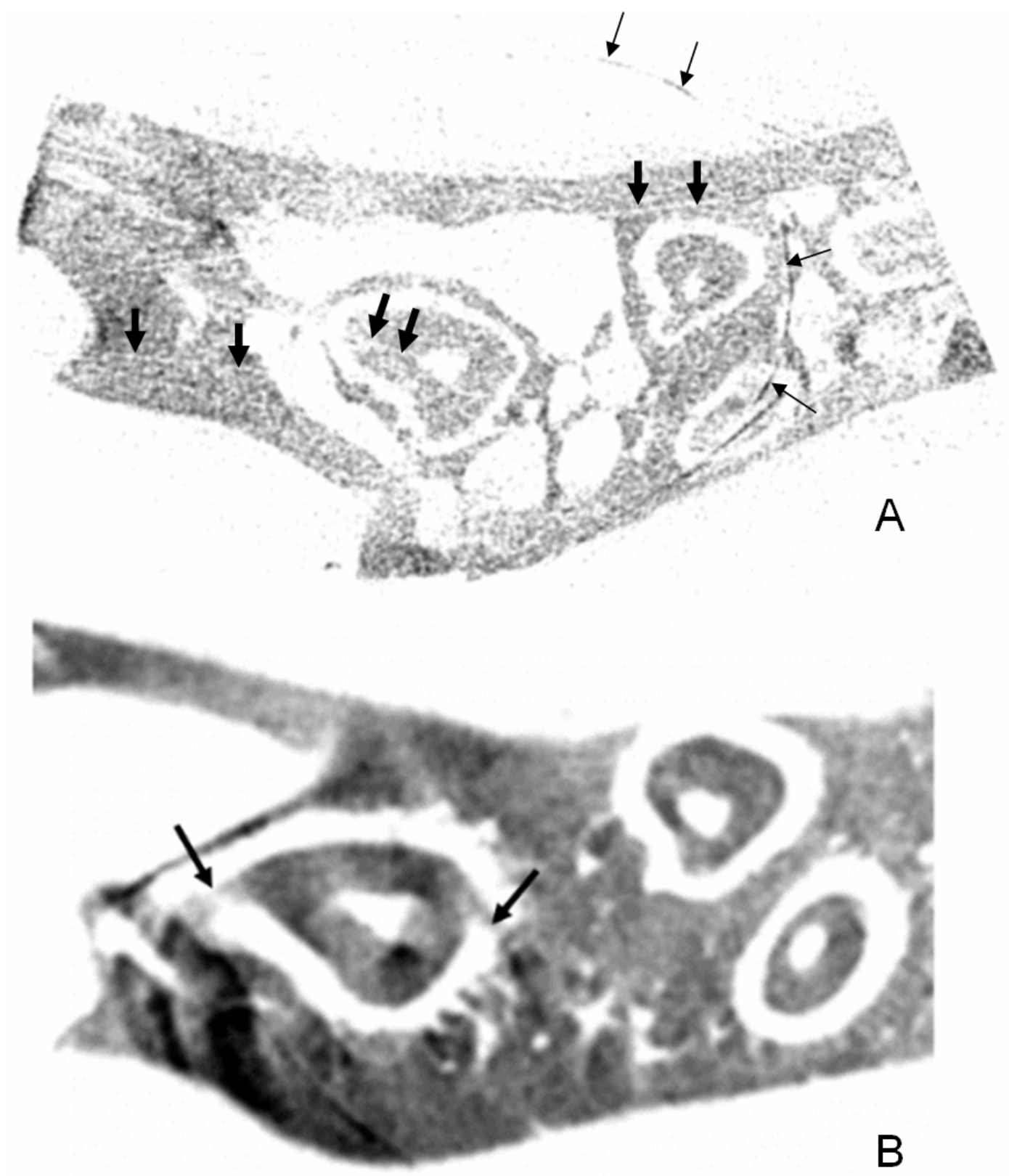

Figure 4. Alignment (wing) artefacts (arrows) on the mesial root of the mouse first molar. 


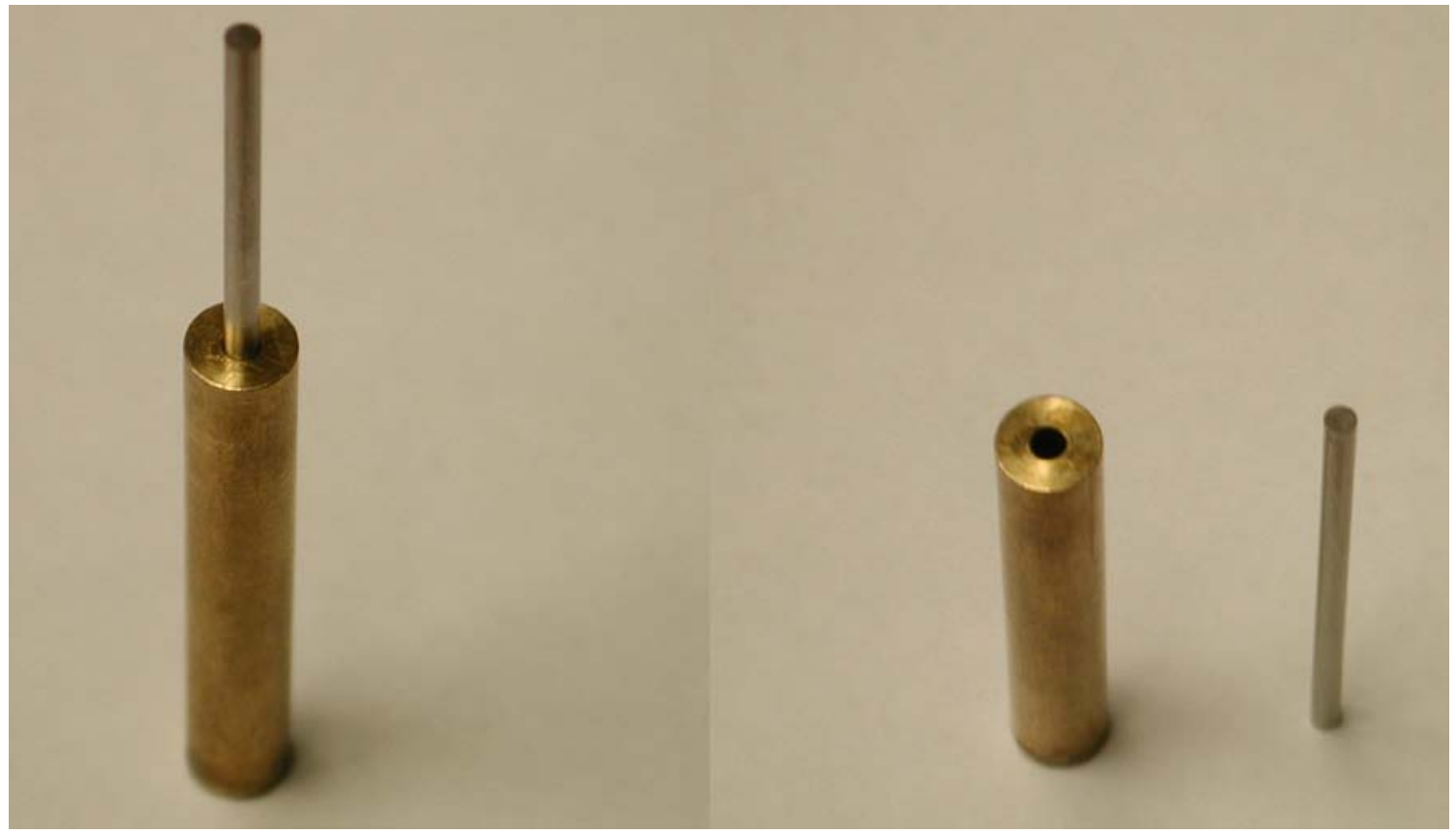

Figure 5. Alignment tool, as used for alignment (left) and with separated cylinders for specimen scanning (right). 


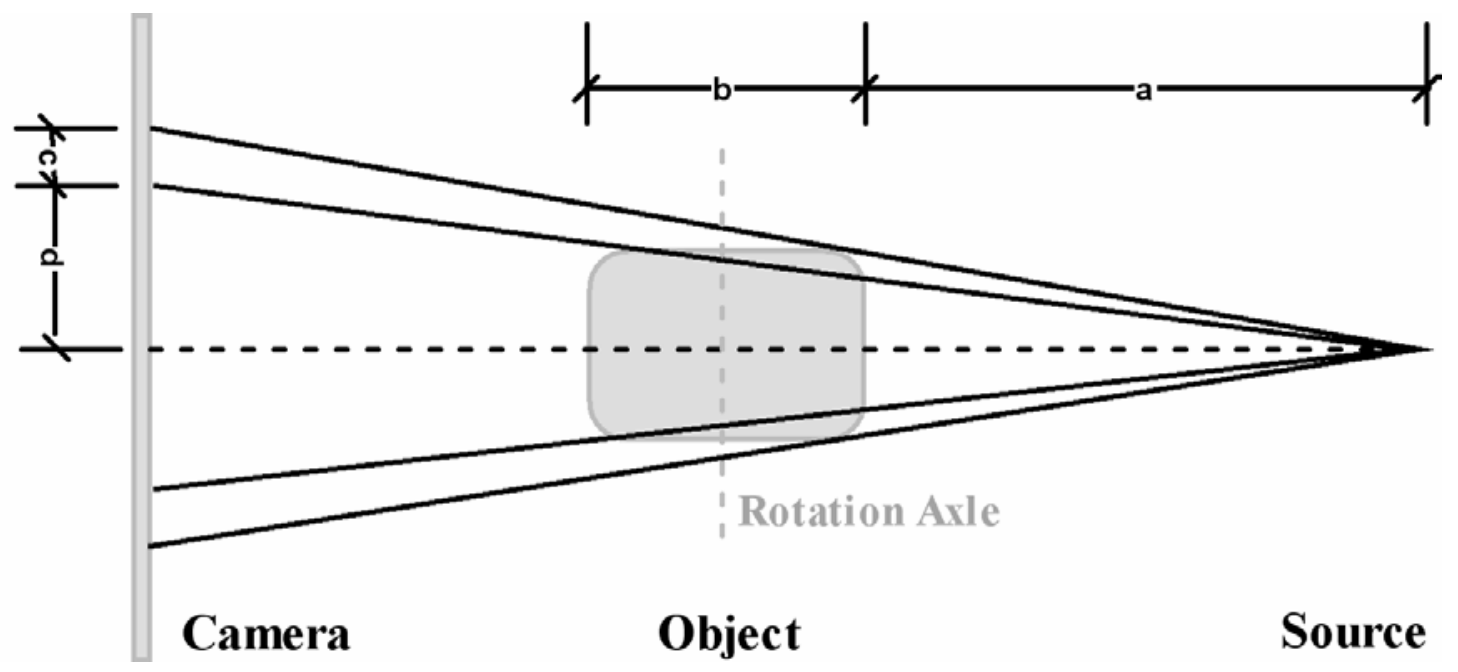

Figure 6. A difference in the object magnification from 0 to 180 degrees scans. The higher the object width $b$ and the higher the magnification, increased by decreasing a, the higher the discrepancy c. 

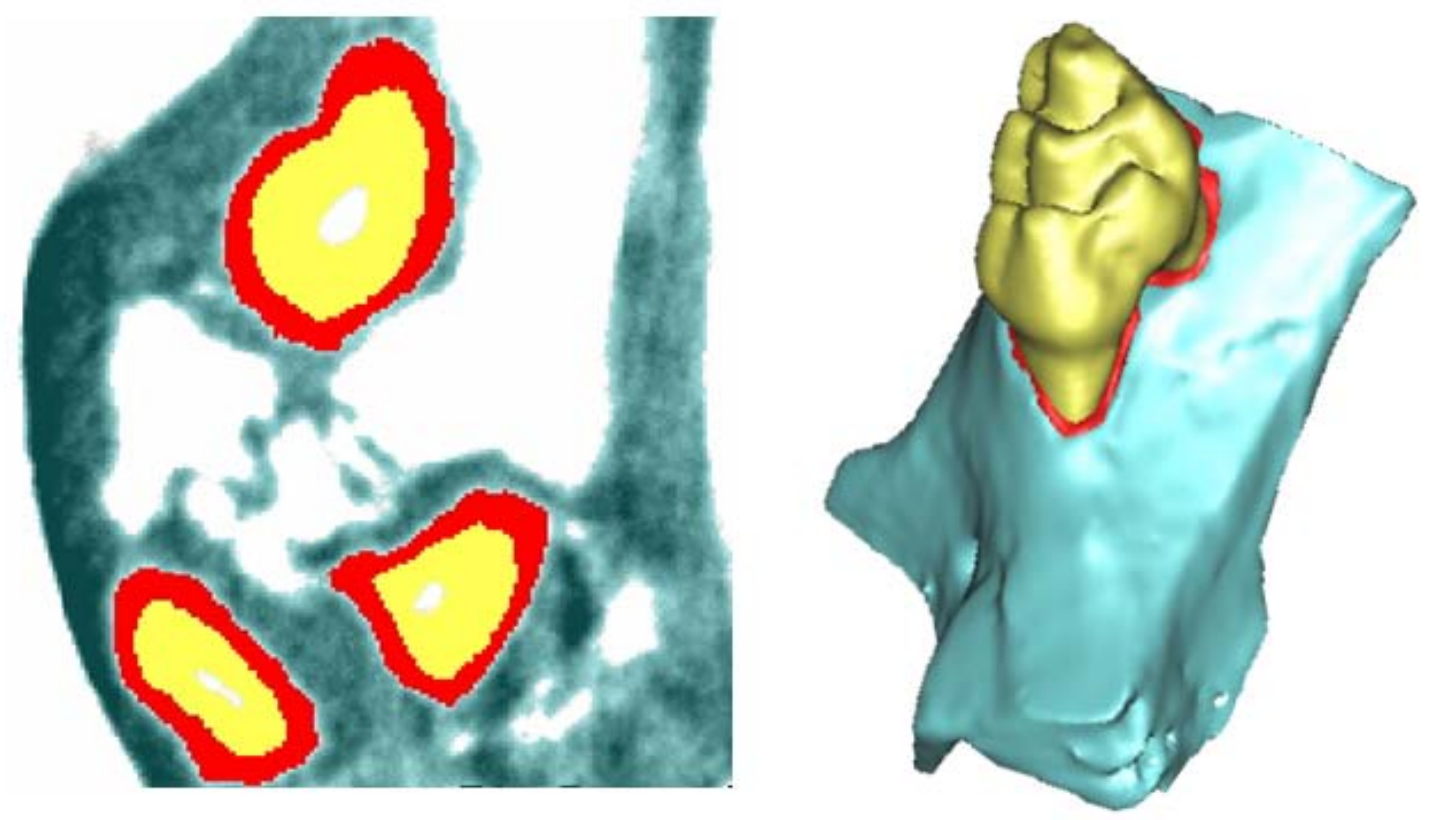

Figure 7. Segmentation of bone, periodontal ligament and tooth into different masks before and after 3D rendering. 

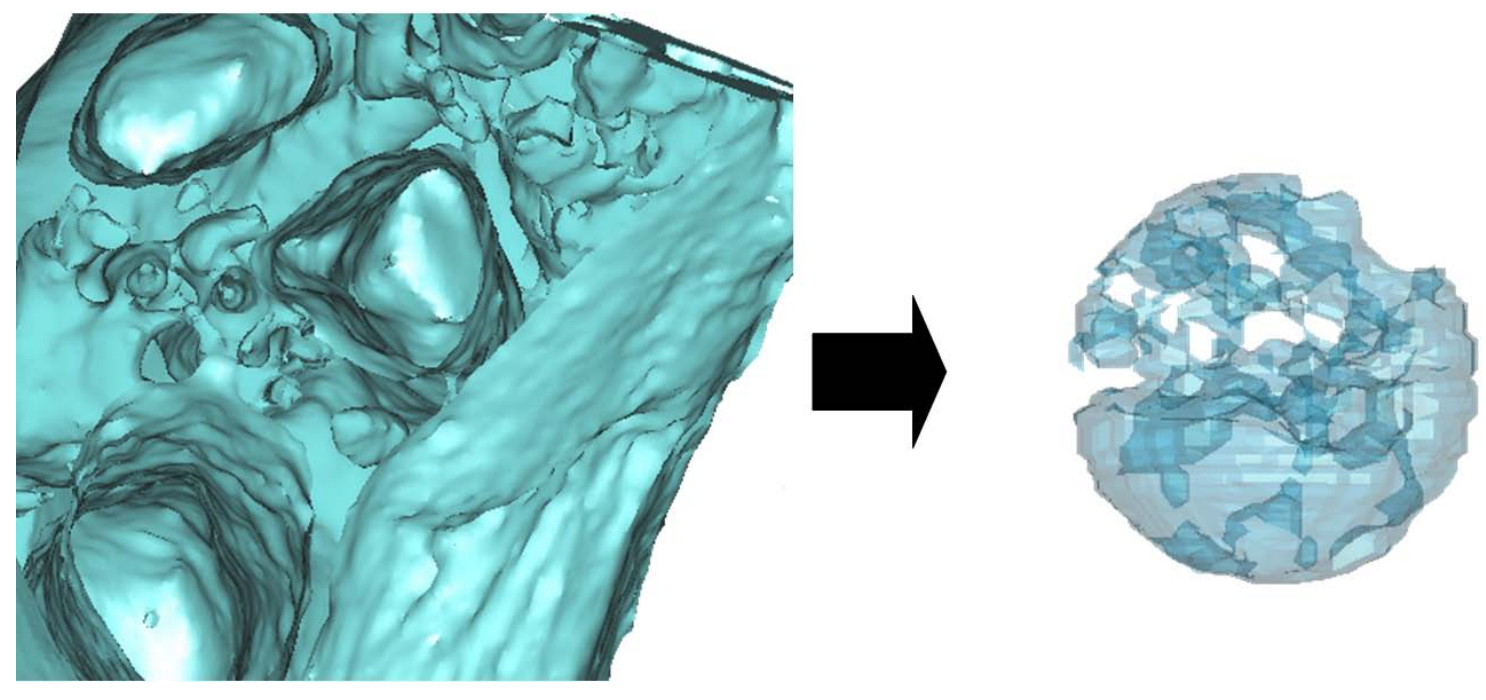

Figure 8. Bone plane cut to show the $0.5 \mathrm{~mm}$ diameter spherical sample from alveolar bone between the roots. Transparency was toggled to allow interior visualization. 

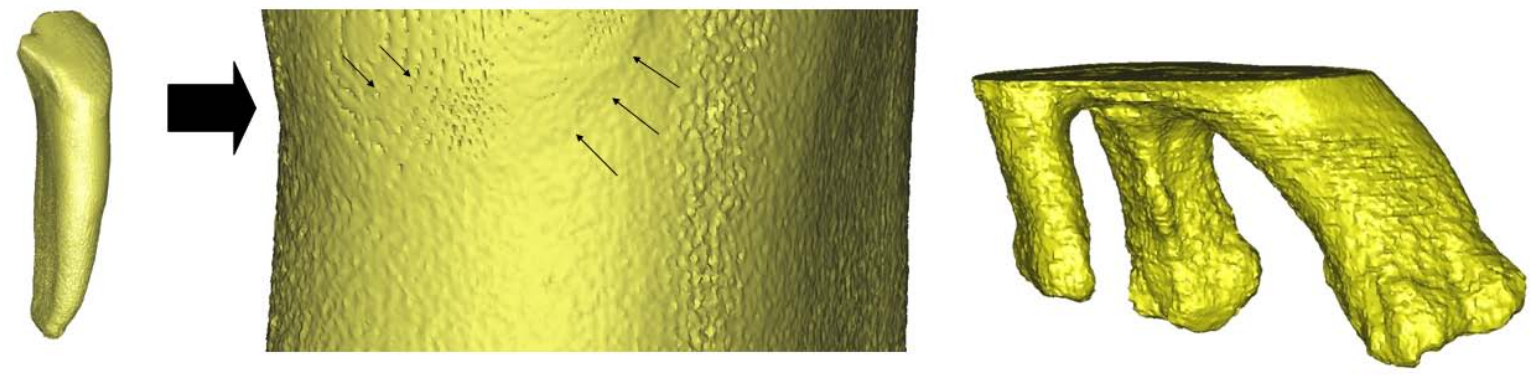

Figure 9. Dog incisor and mouse molar root rendered with maximum accuracy. A close up to visualize Retzius striae, cementum-enamel junction and root surface roughness in the dog tooth is also included. 

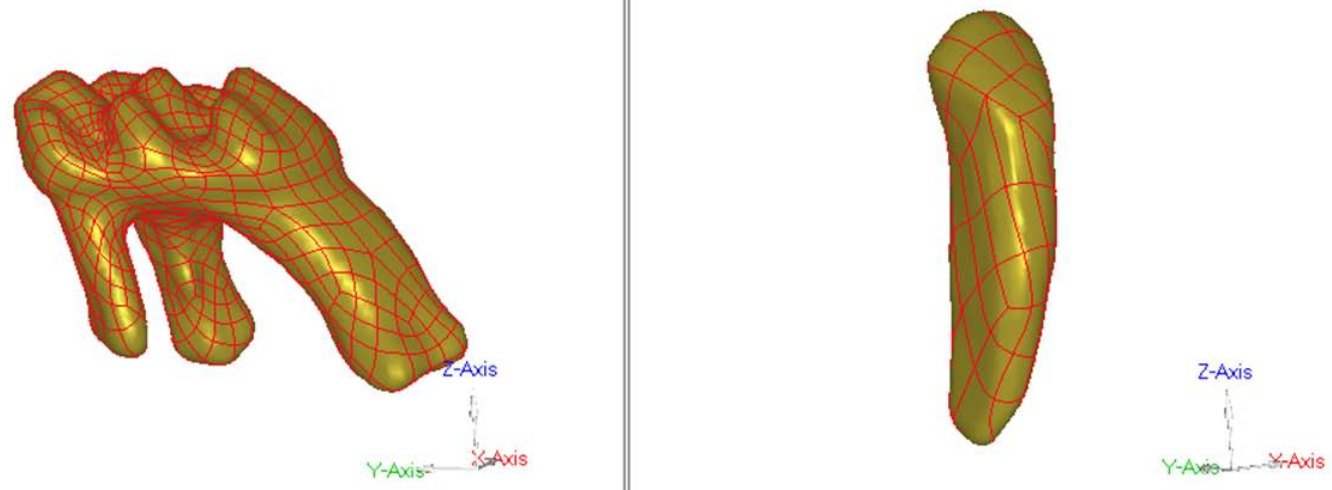

Figure 10. Examples of solid models generated after editing the stl files. Smoothing was performed to allow quality meshing, but the main features of the original scan were maintained. 


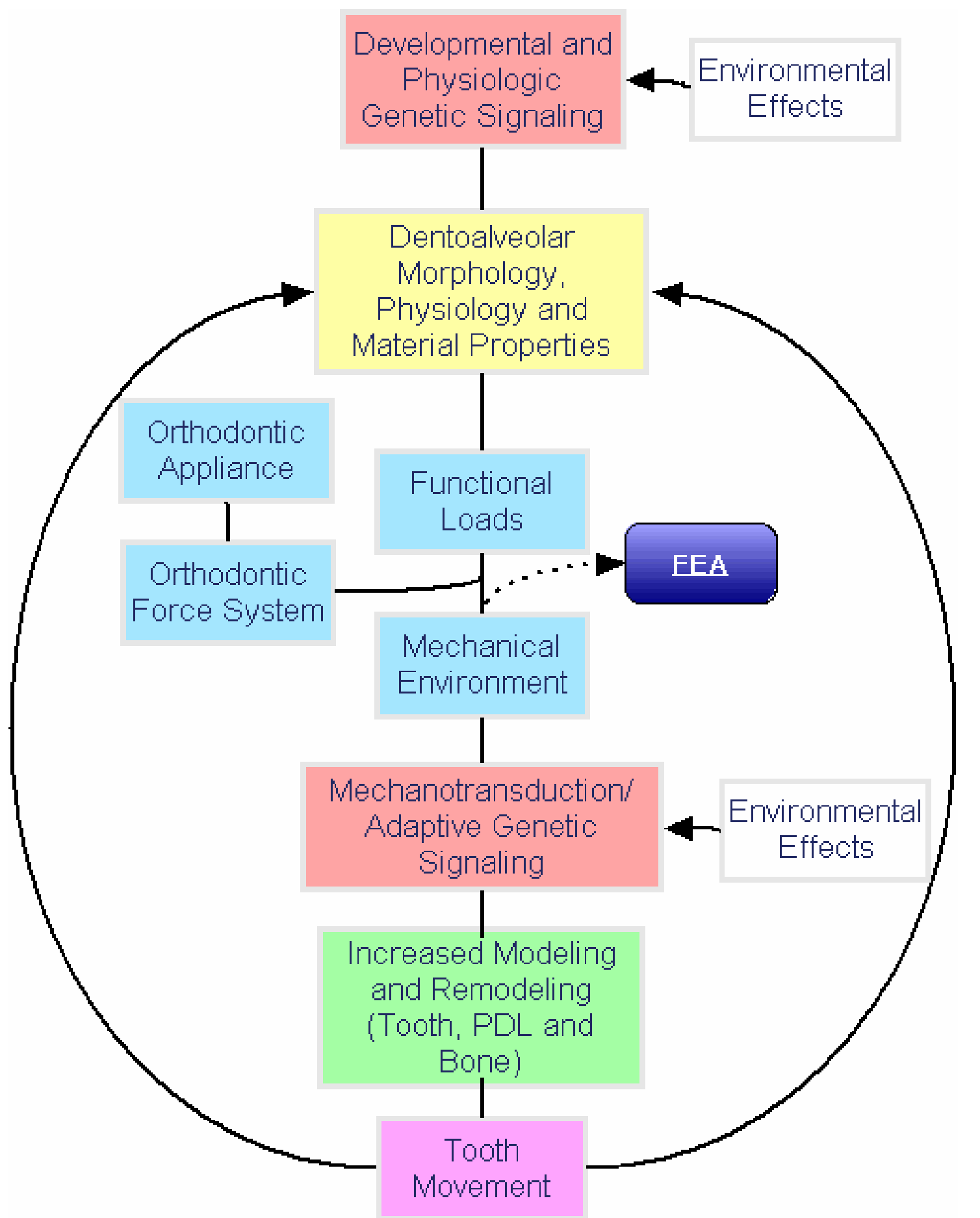

Figure 11. Diagram of contemporary orthodontic biomechanics. 

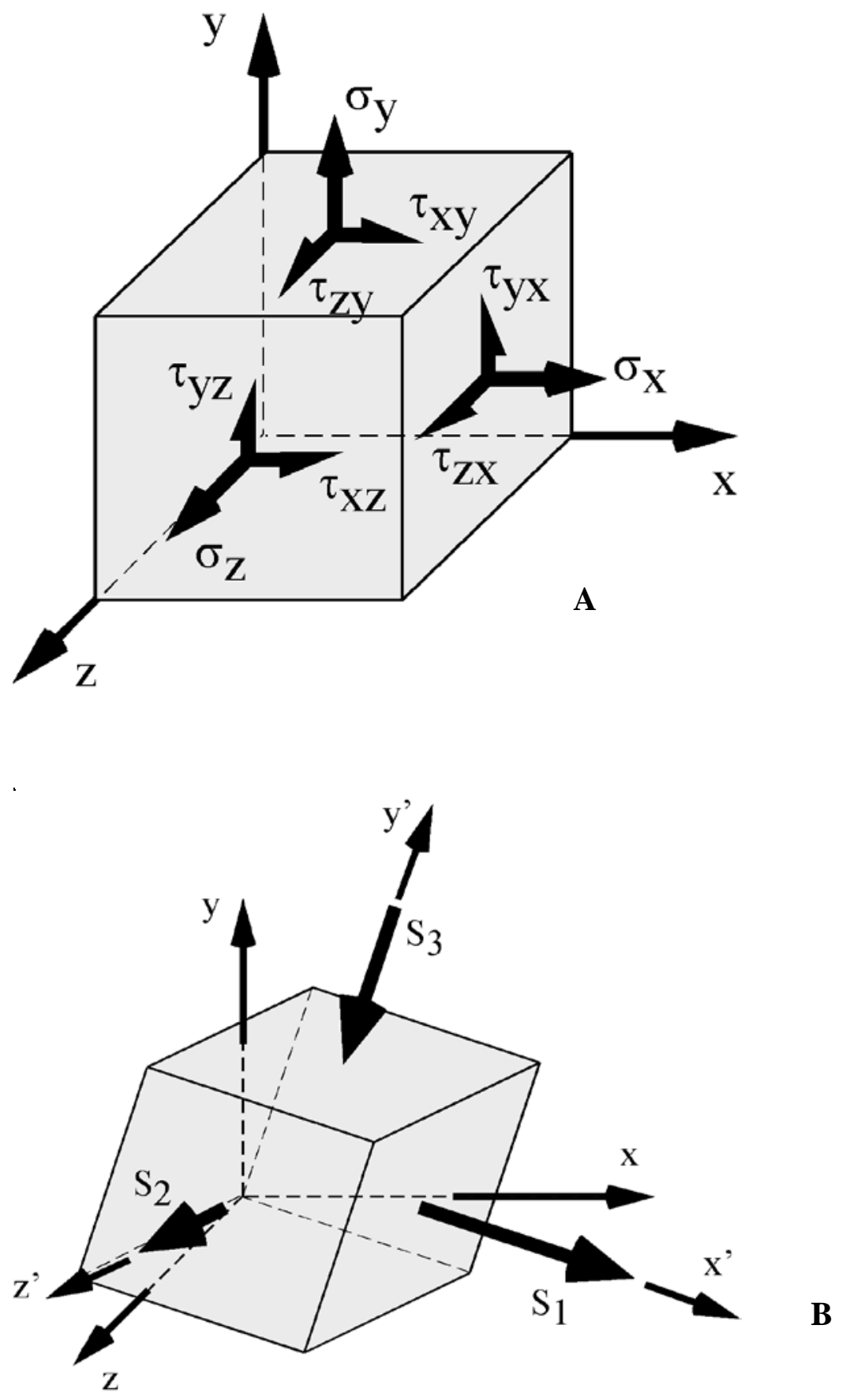

Figure 12. A, General state of stress; B, Principal stresses in the principal (x’, y’, z’) directions. 


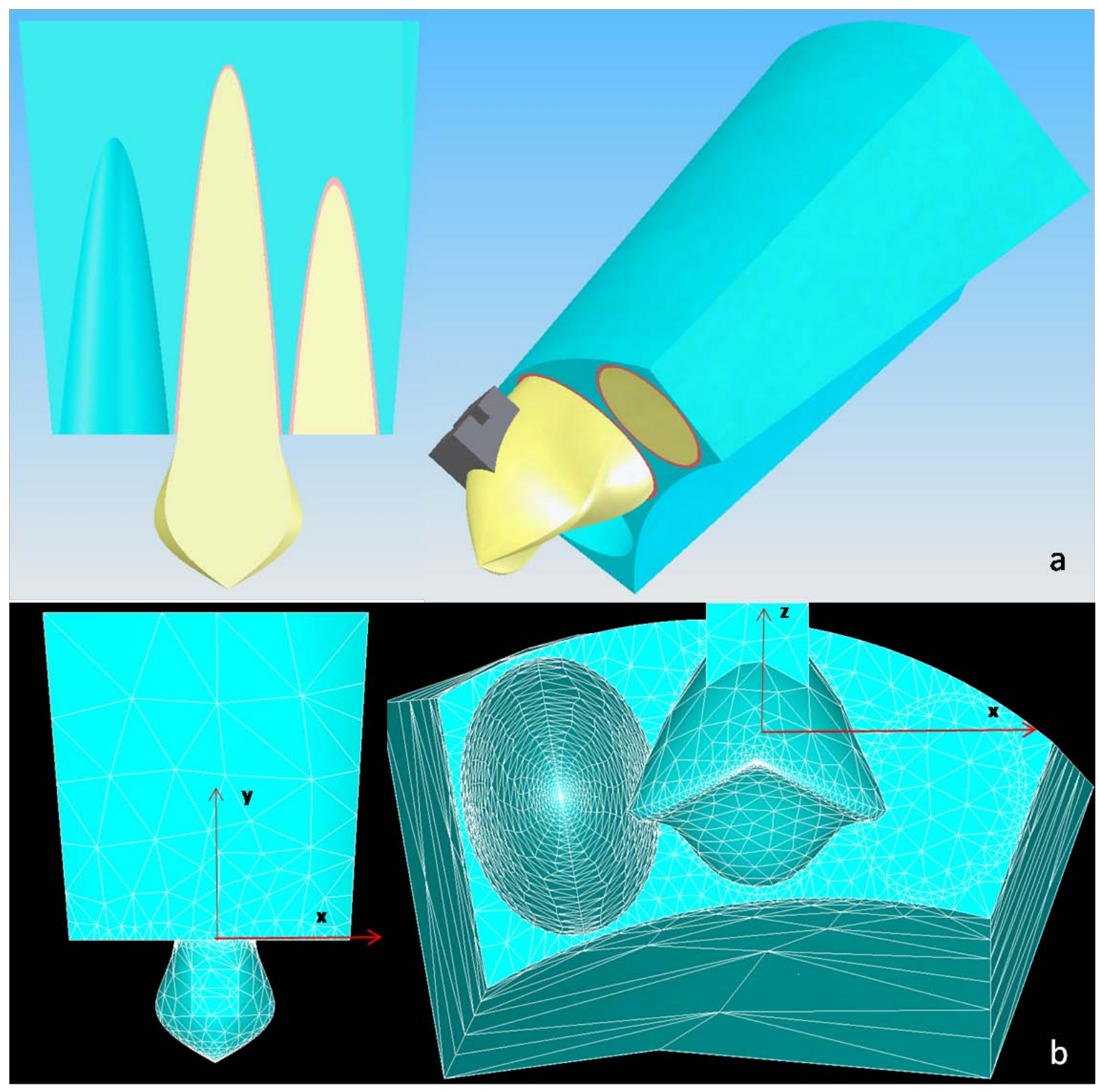

Figure 13. A, CAD model showing internal morphology; B, FE model and coordinate system. 

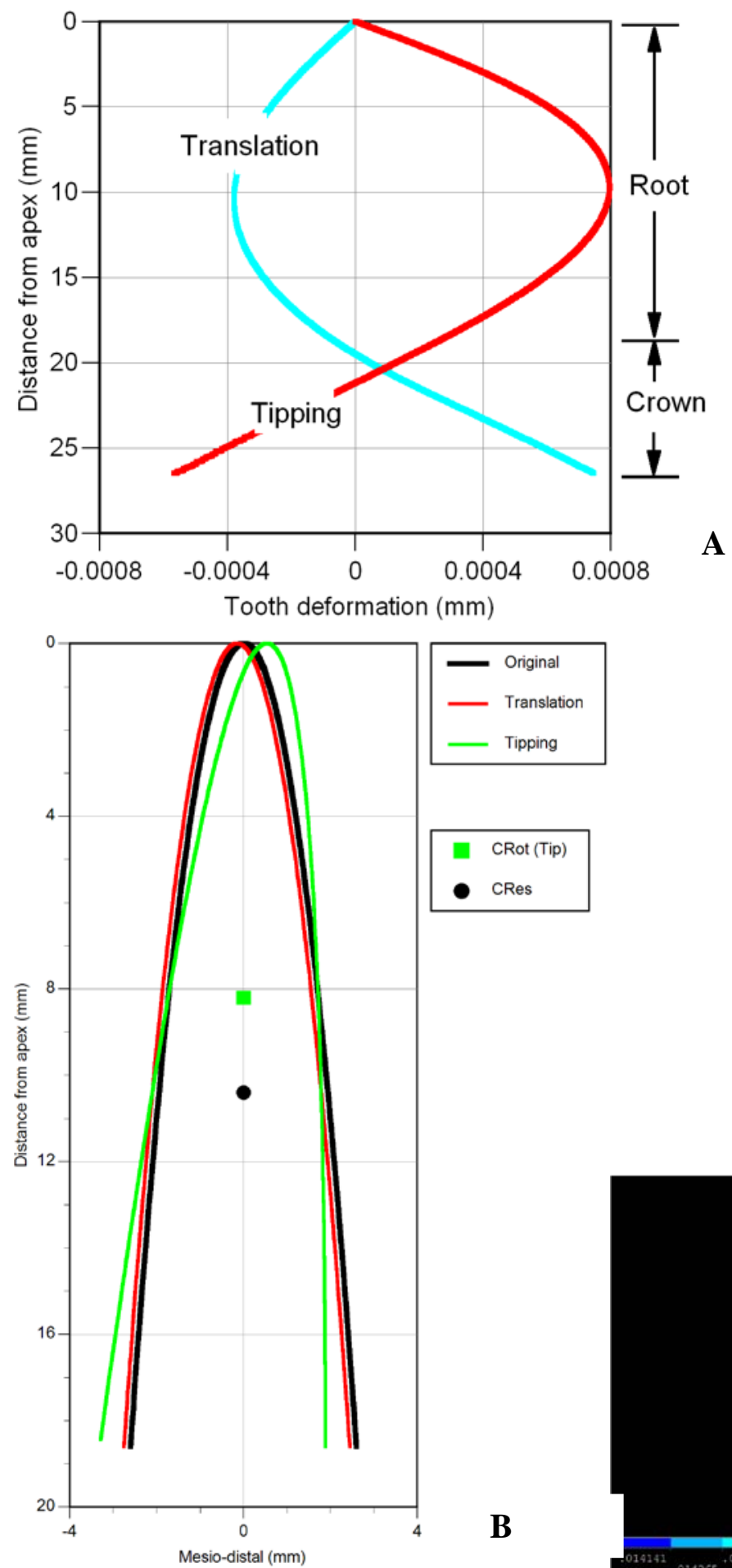

B

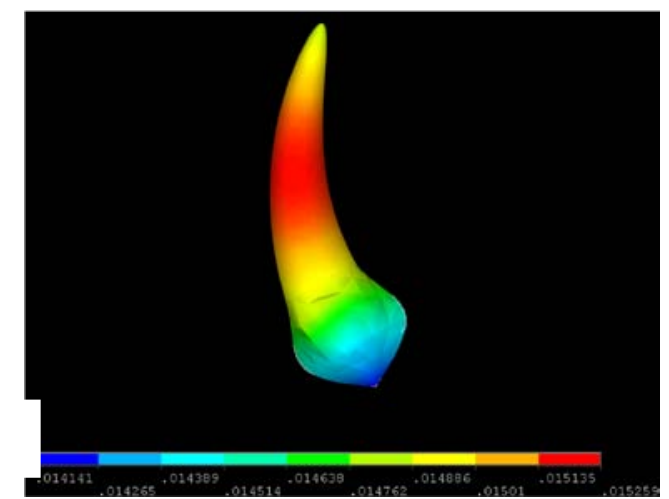

C

Figure 14. A, Exaggerated (10x) displaced positions of the root. B, Deformed root shapes. C, Exaggerated (x5000) total tooth displacement in translation. The scale is in $\mathrm{mm}$. 


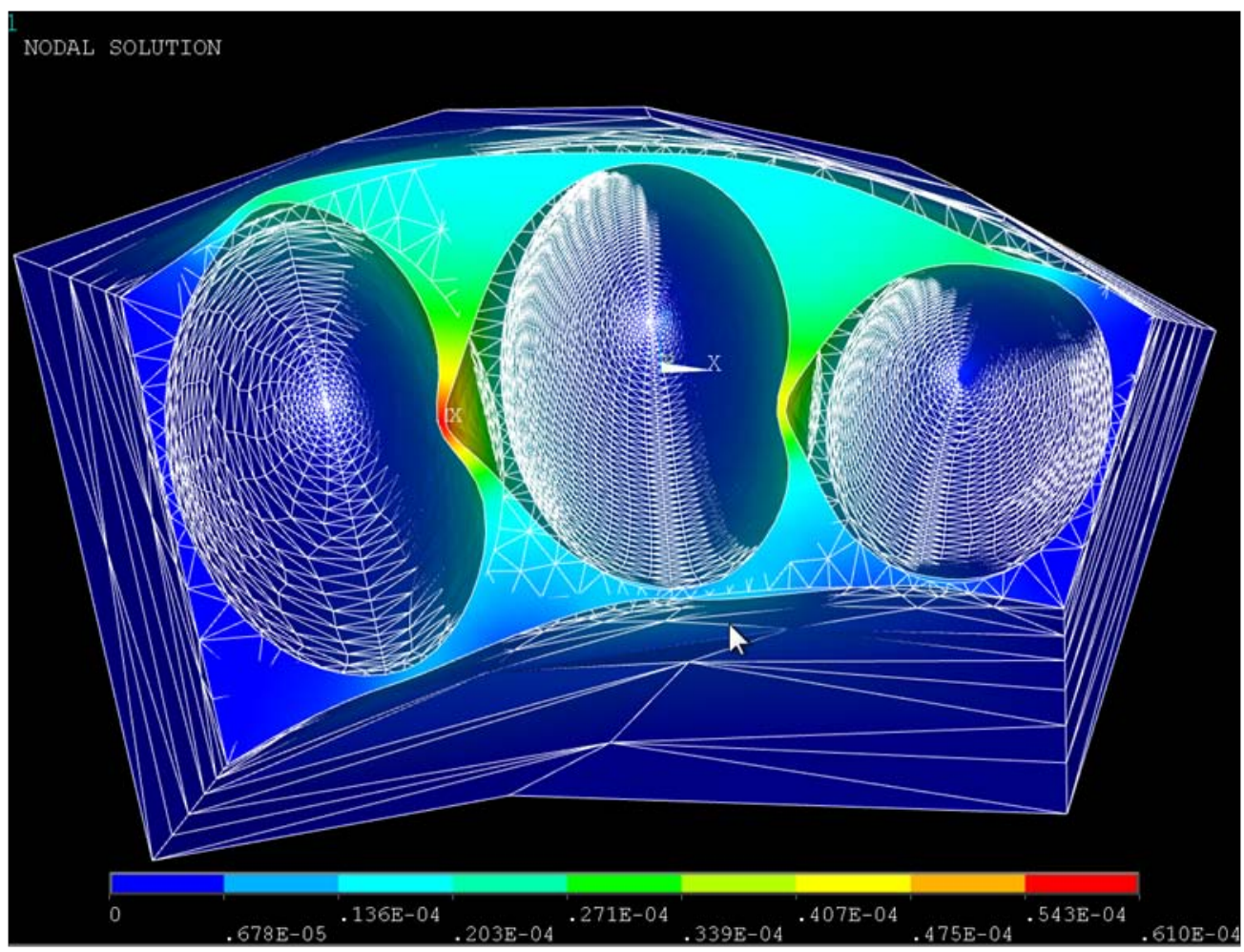

Figure 15. Exaggerated 3-D occlusal view of socket deformations resulting from tooth translation. The final position is the colored solid and the initial position is the element skeleton. 

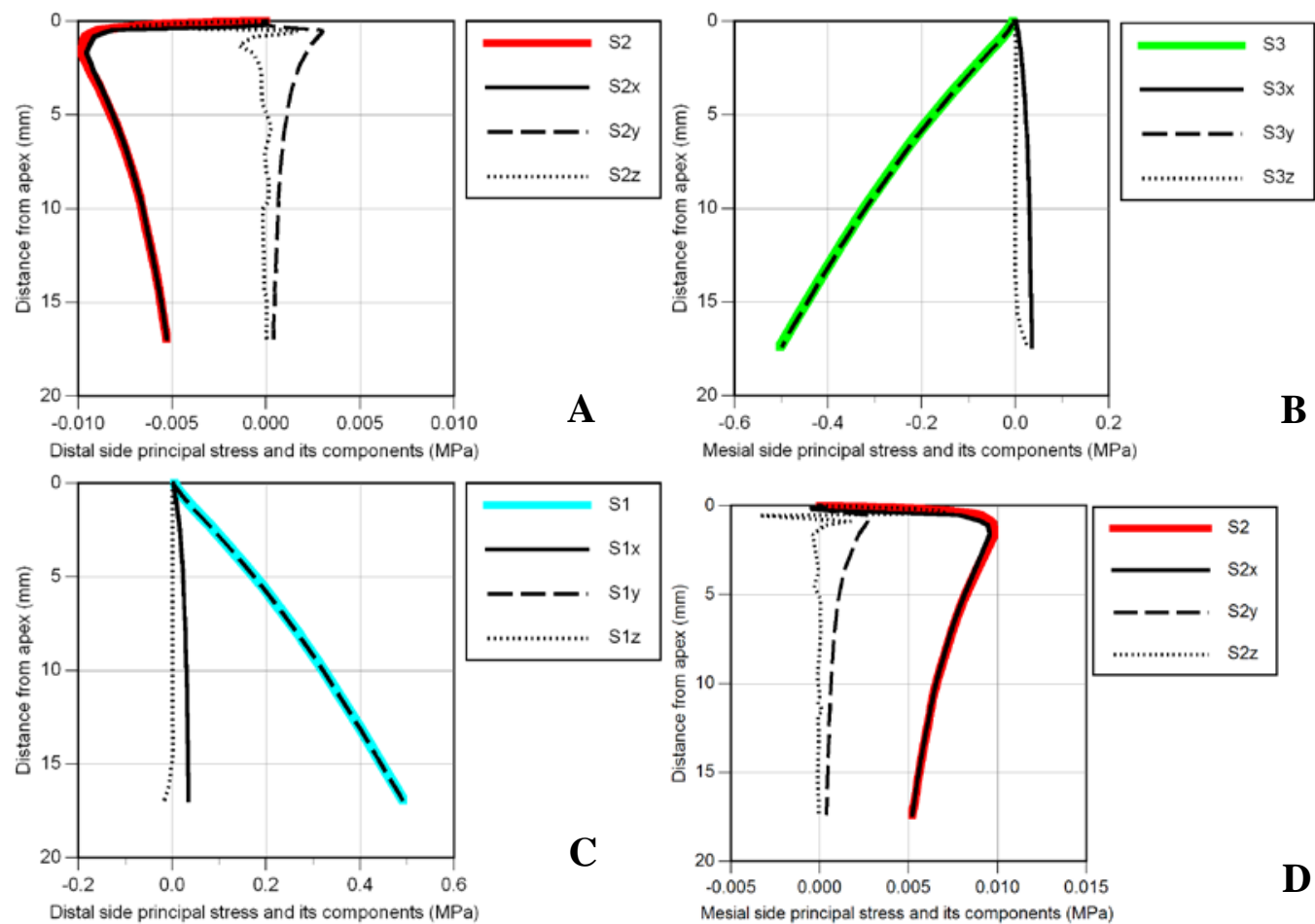

Figure 16. Tooth translation associated principal stresses in the root: A, distal side S1 (S3 is almost zero;) B, mesial side S3 (S1 is almost zero;) C, distal side S2; D, mesial side S2 

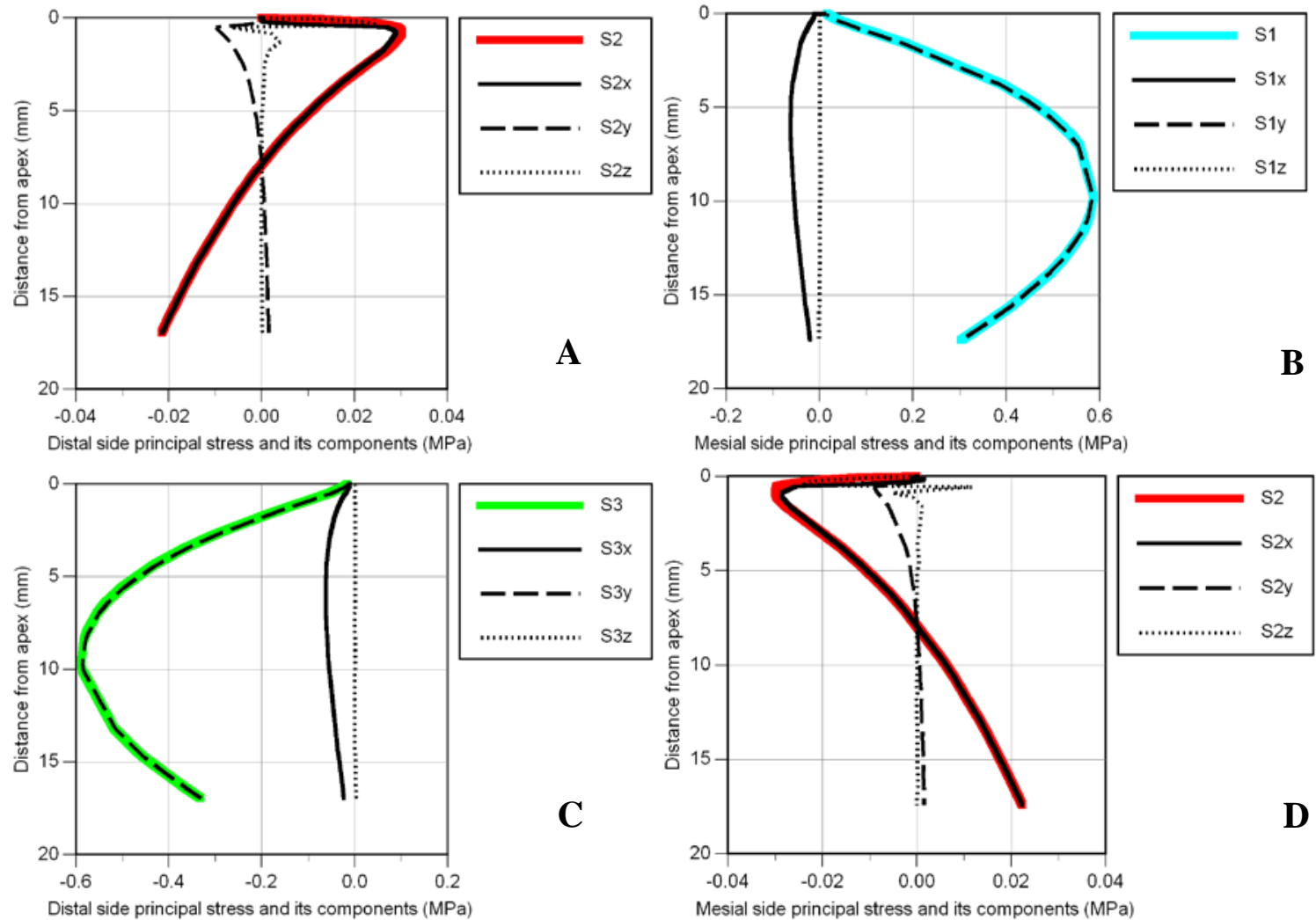

Figure 17. Tooth tipping associated principal stresses in the root: A, distal side

S3 (S1 is almost zero); $\mathbf{B}$, mesial side S1 (S3 is almost zero); $\mathbf{C}$, distal side S2;

D, mesial side S2. 

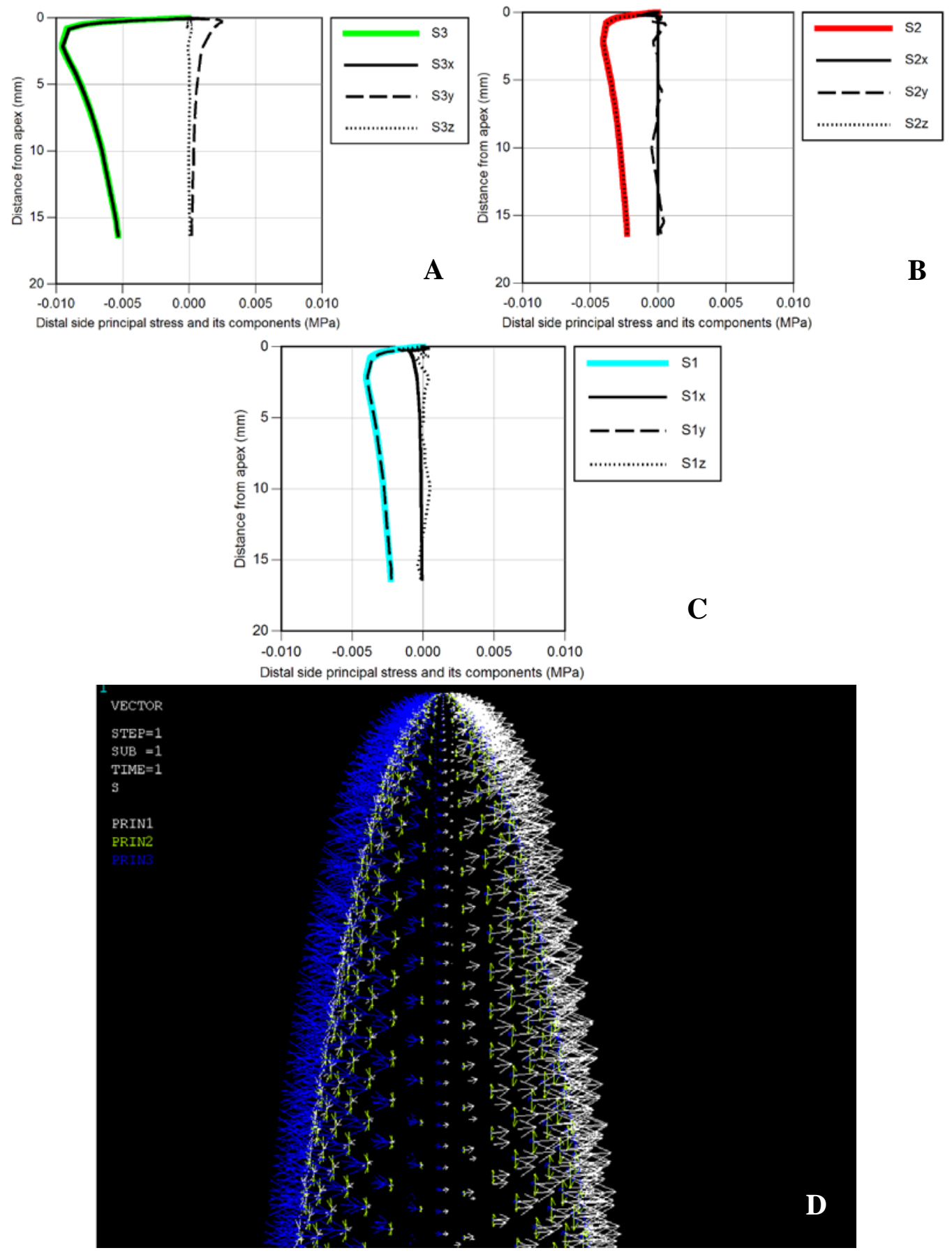

Figure 18. Tooth translation associated principal stresses and their components on the distal side of the PDL: A, S1; B, S2; C, S3. D, buccal view of PDL principal stresses in the apical $\sim 1 / 3$ of the root during translation (left side is the distal side). 

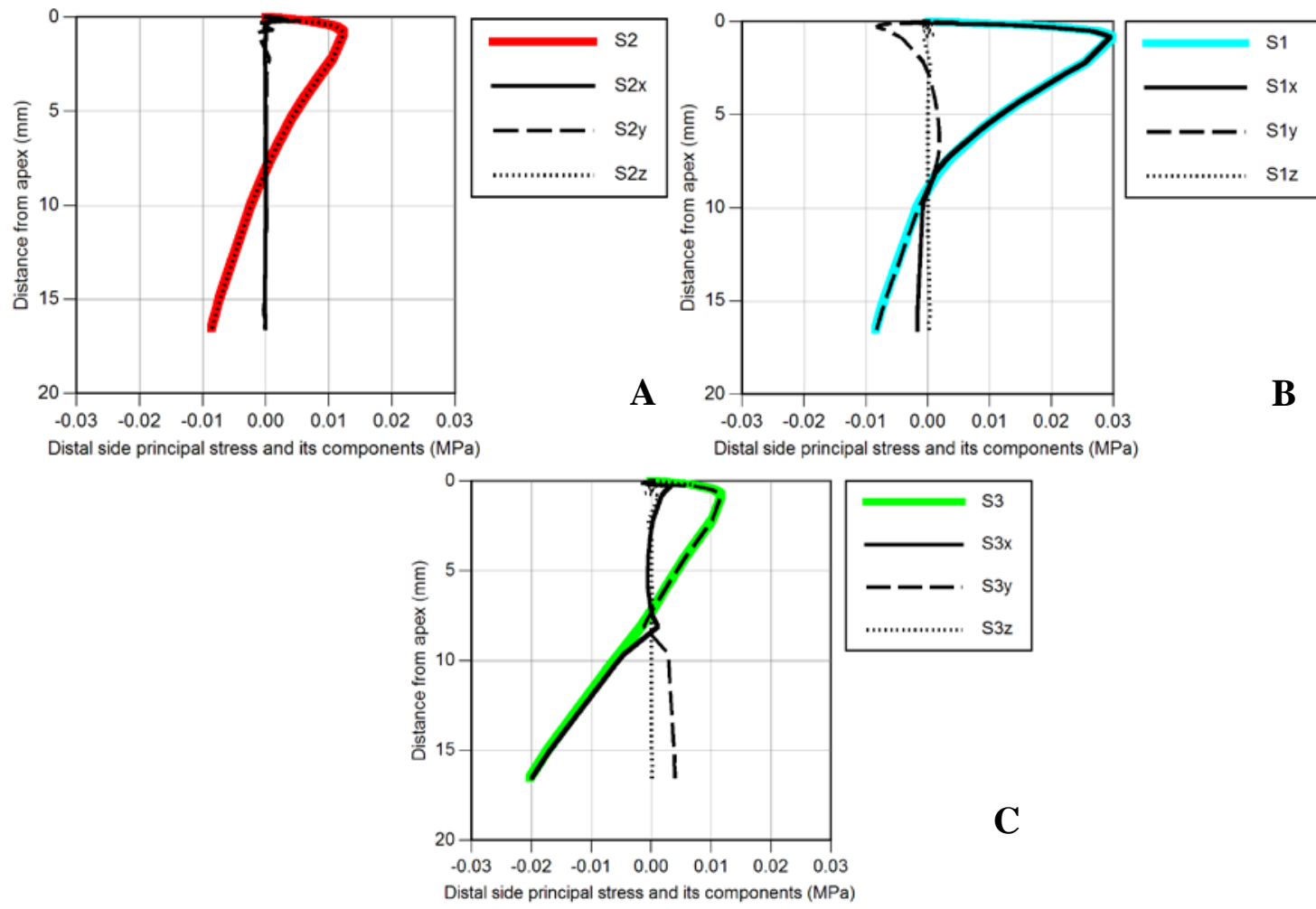

Figure 19. Tooth tipping associated principal stresses and their components on the distal side of the PDL: A, S1; B, S2; C, S3. 


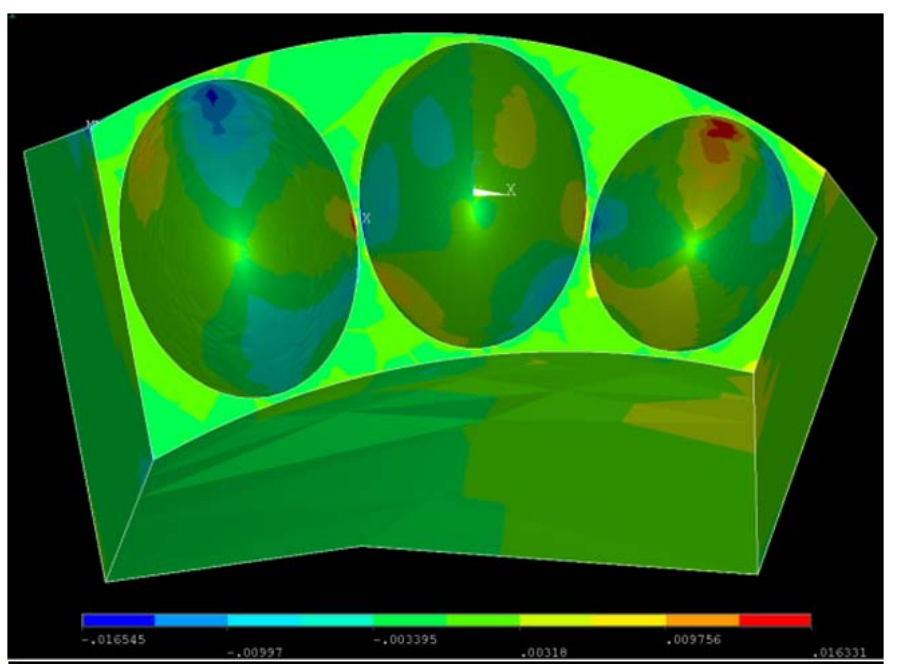

A

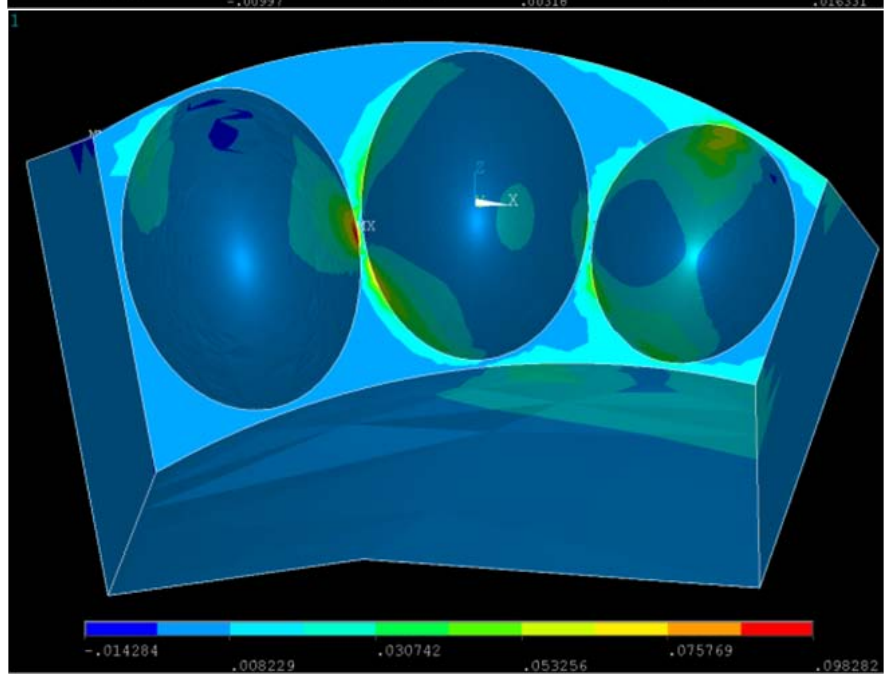

B

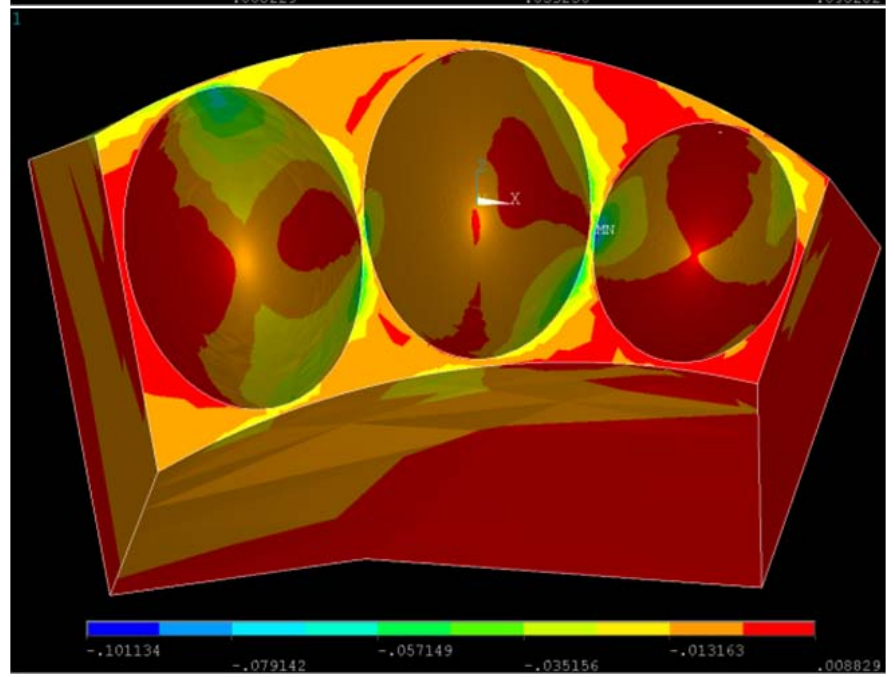

C

Figure 20. Three-dimensional magnitude plot of tooth translation associated principal stresses in bone. A, S1; B, S2; C, S3. 

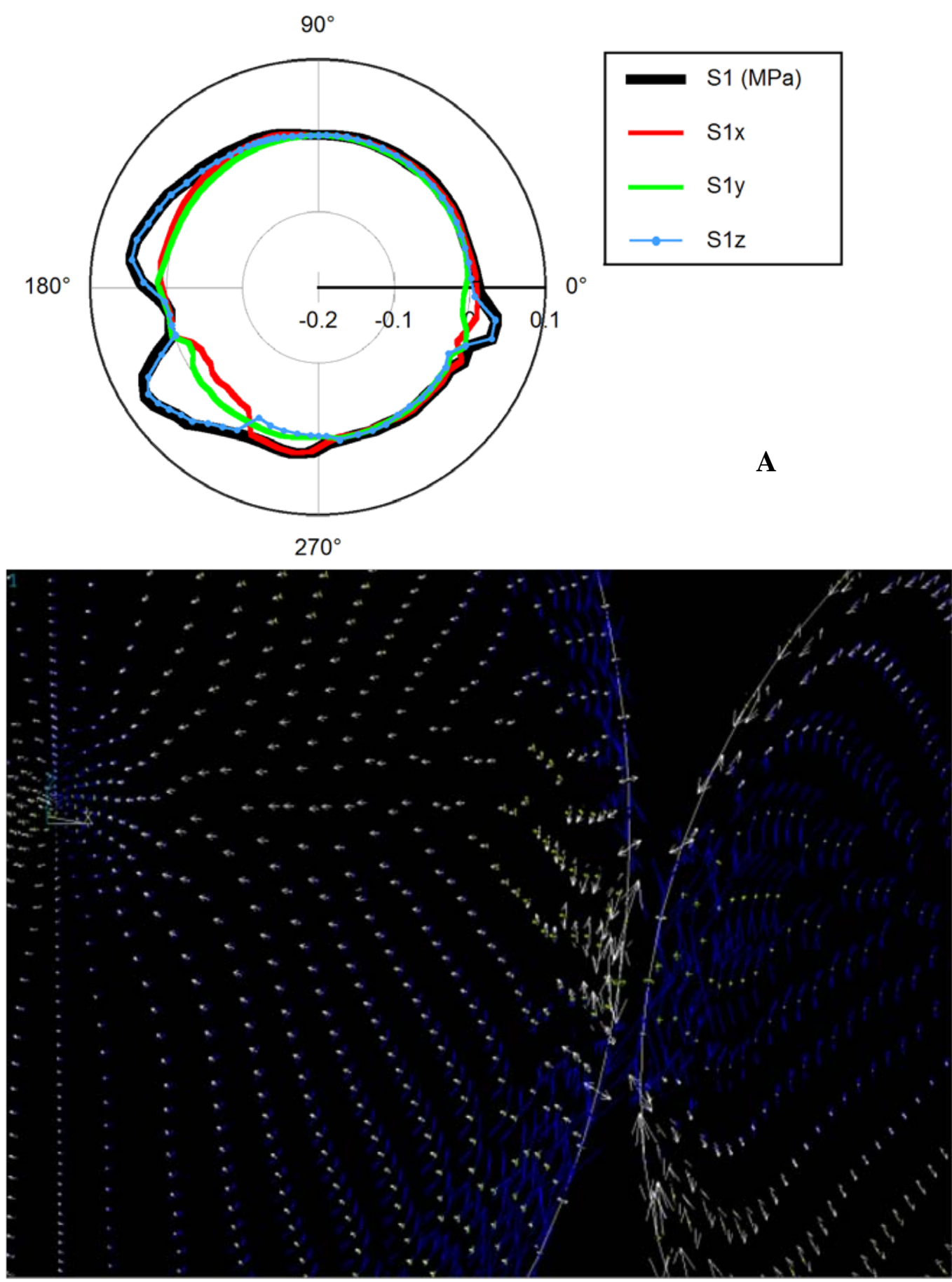


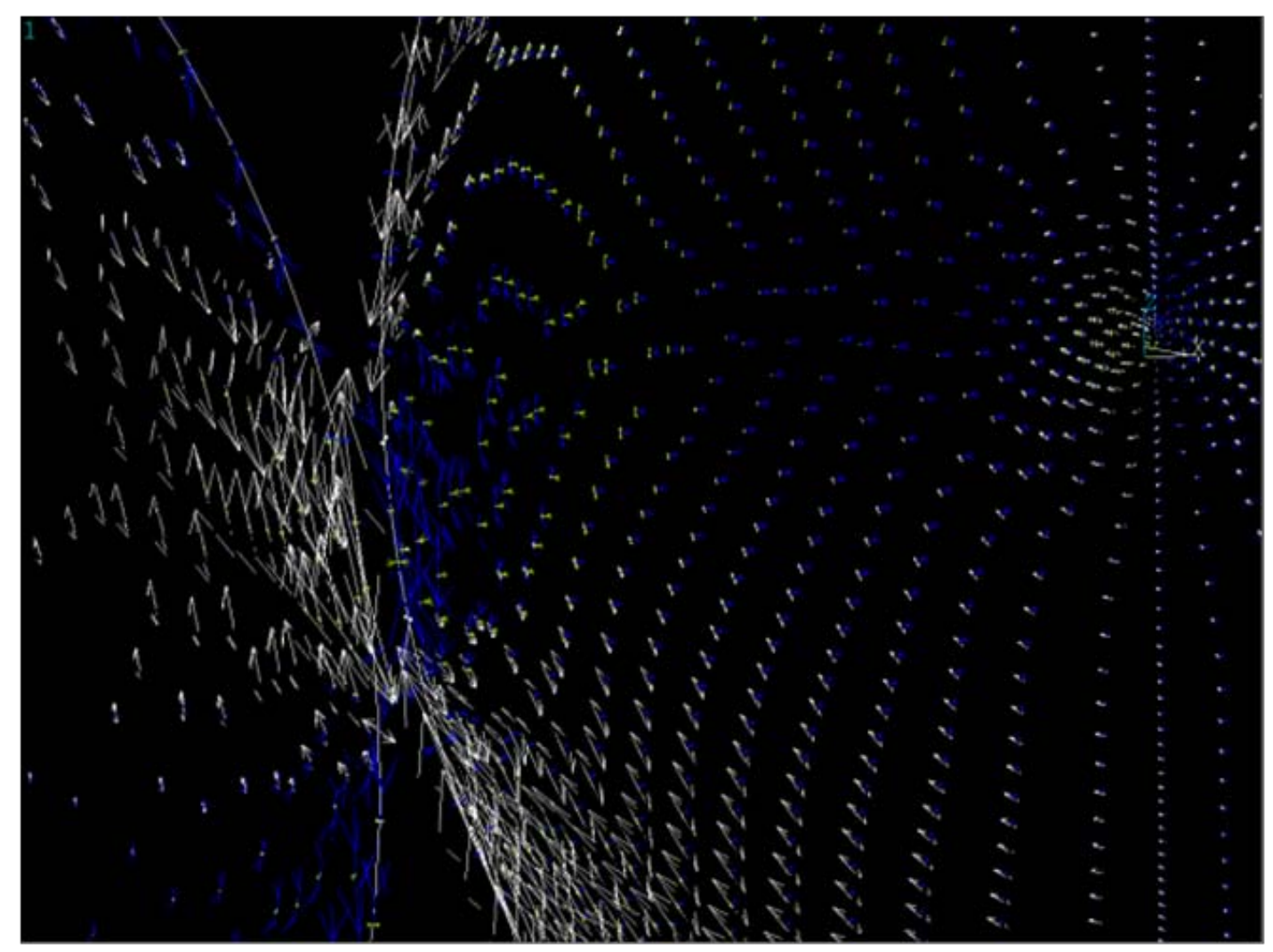

C

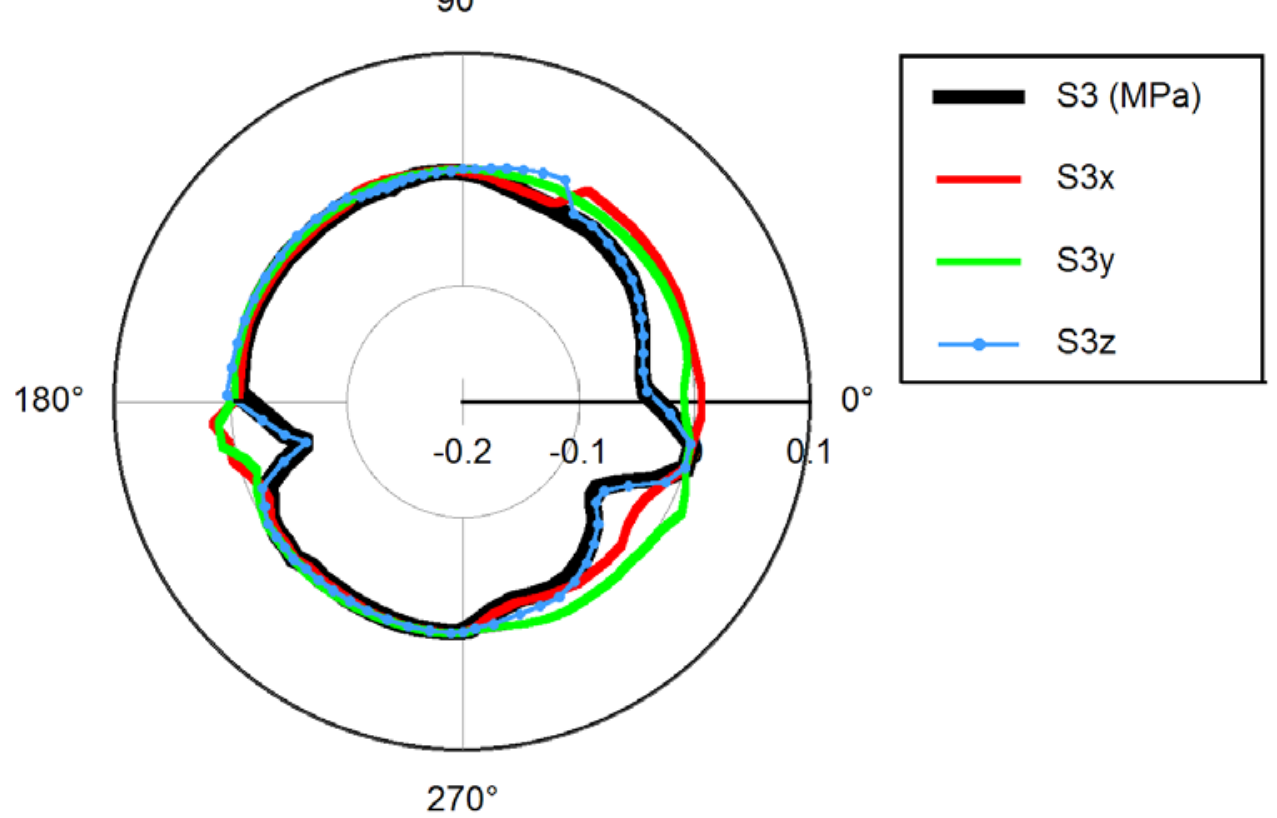

Figure 21. 3-D direction plots of tooth translation associated principal stresses in bone, $\mathbf{A}$, distal side (cuspid socket is on the right) and $\mathbf{B}$, mesial side (cuspid socket is on the left;) Polar plots at the cervical region of the bone for $\mathbf{C}$, S1 and D, S3. S2 is not shown because it is very small. 

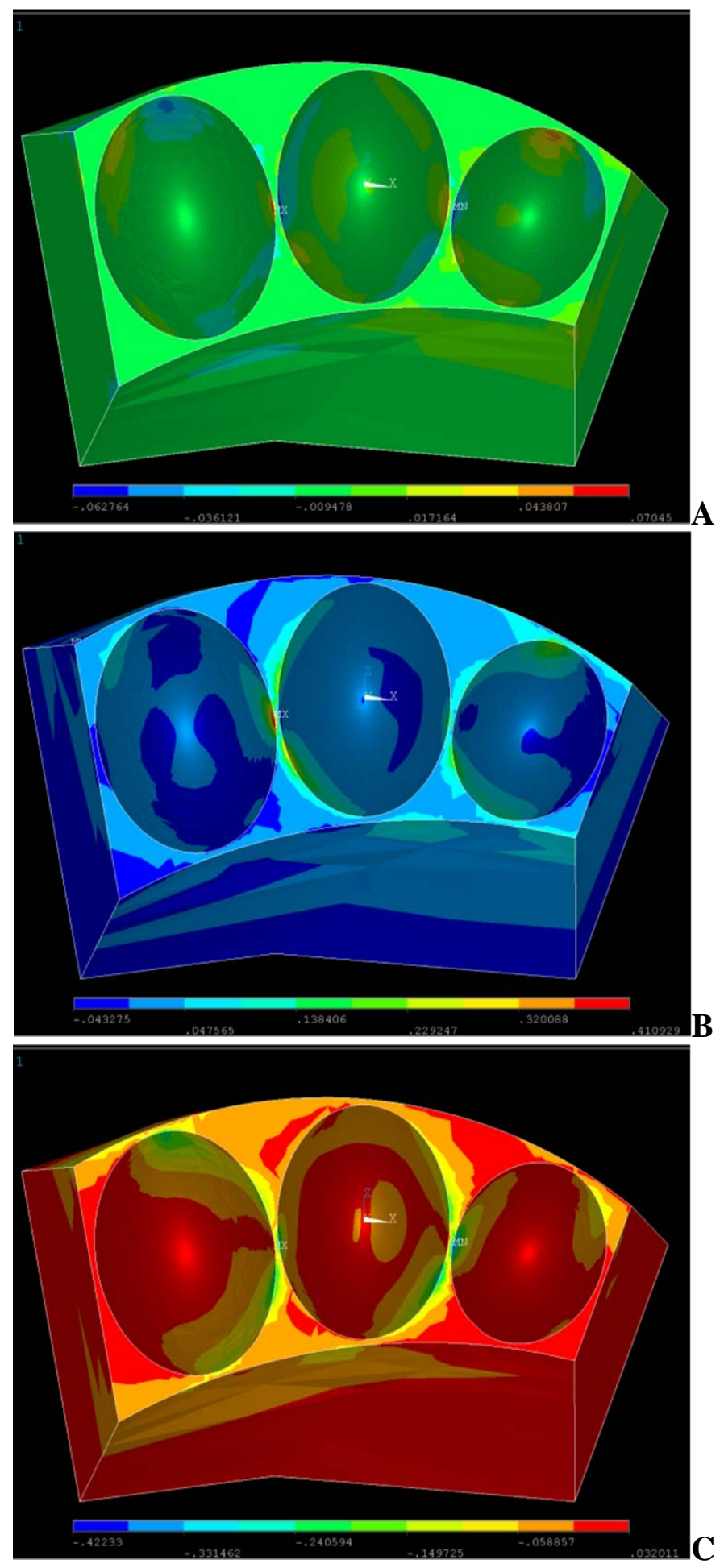

Figure 22. As in Figure 10 except for tooth tipping. 

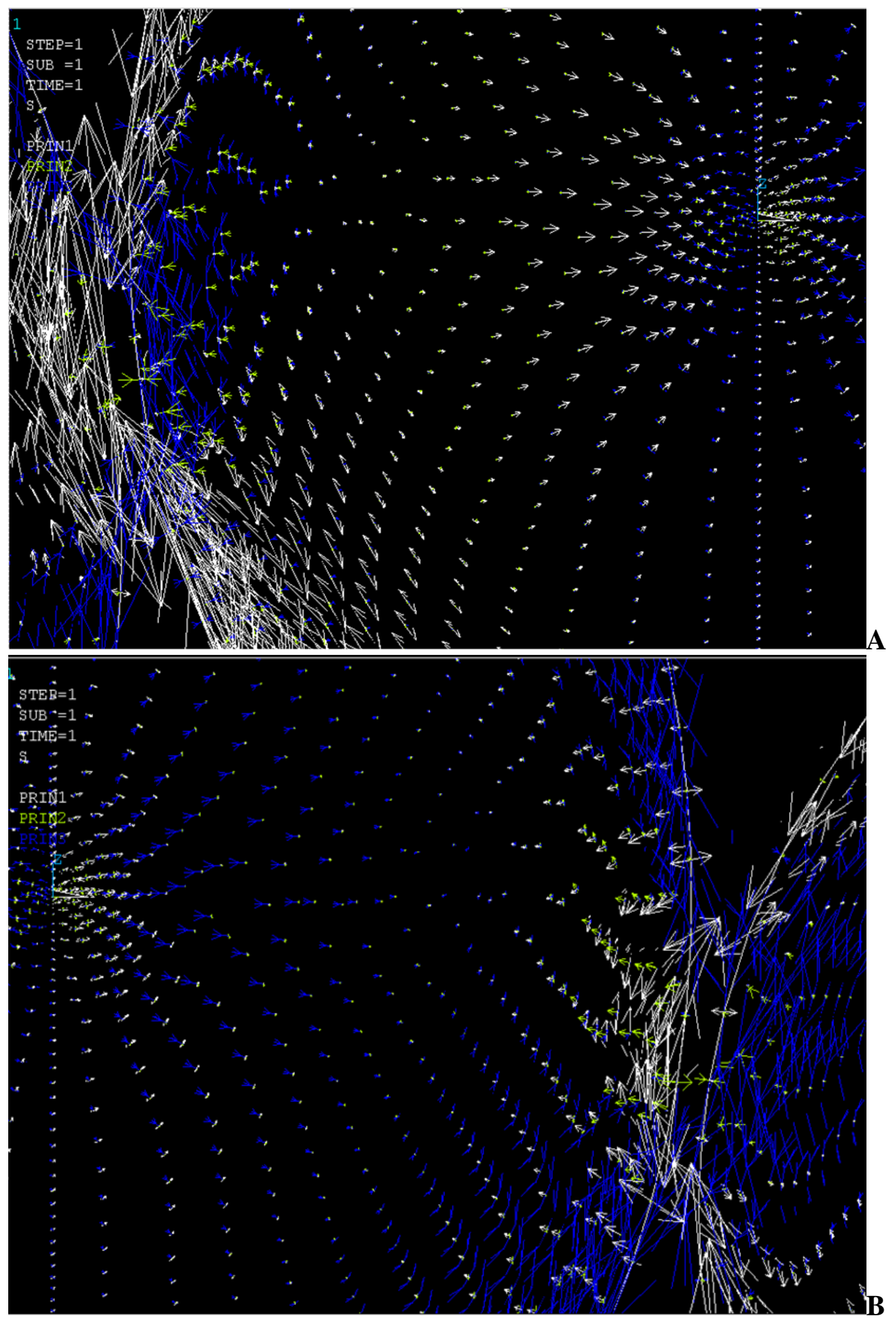

Figure 23. As in Figure 11 except for tooth tipping. 


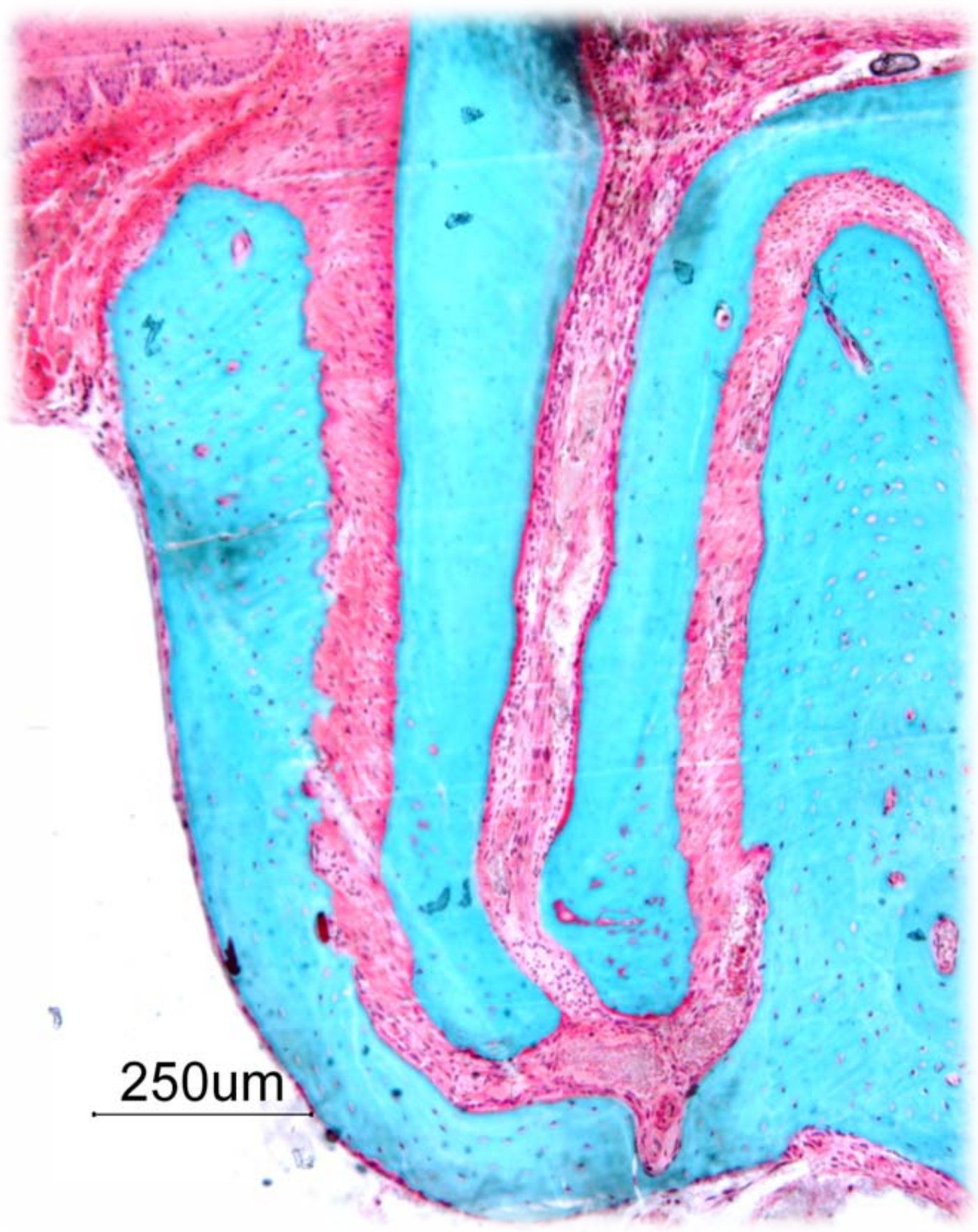

Figure 24. Typical histological section, focusing on the root and PDL. Bone and tooth root are stained blue, and PDL is stained pink.There were no noticeable differences between WT and KO sections. 


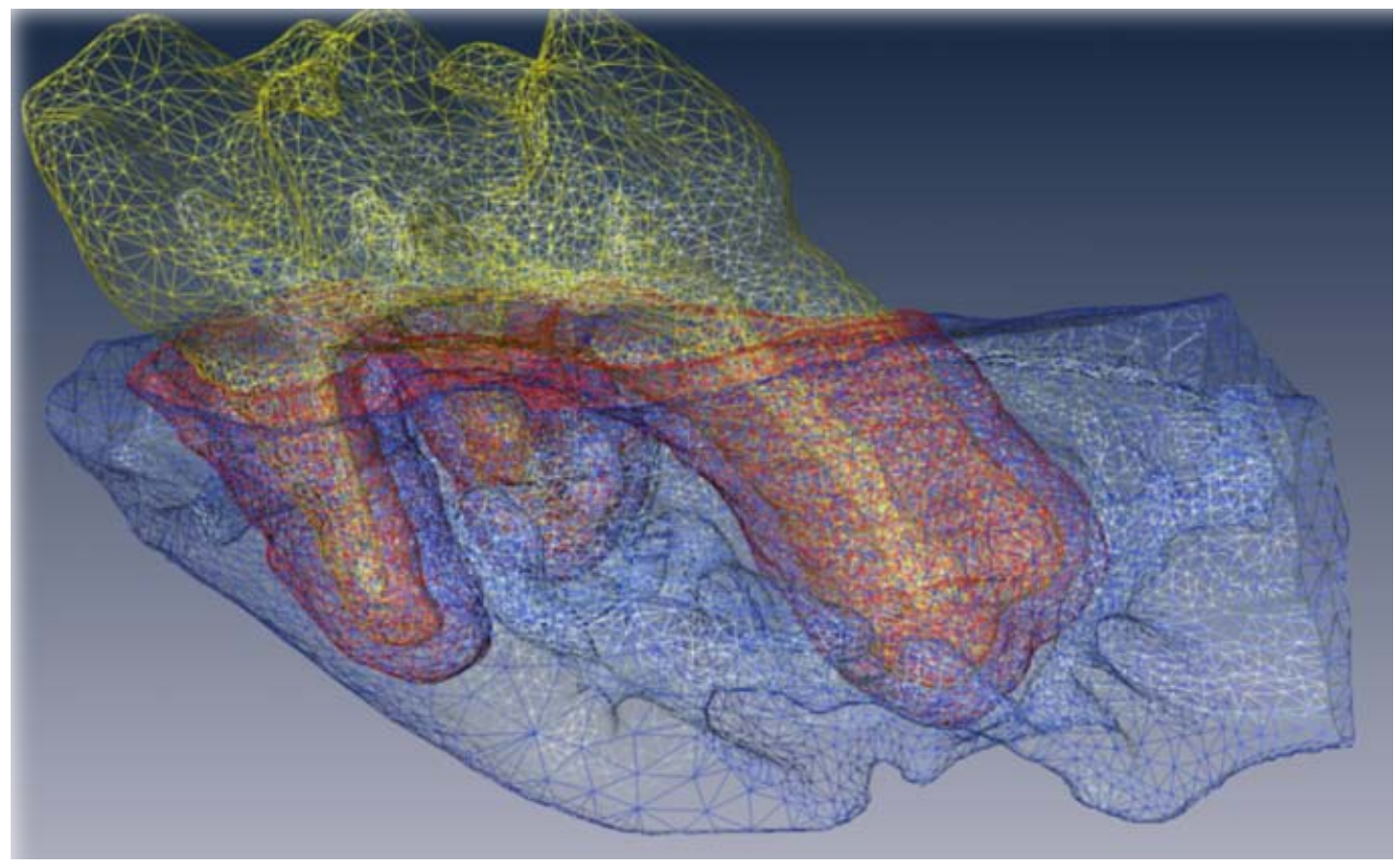

Figure 25. Surface finite element model of a representative specimen showing highly organic internal structure, even after smoothing of tissues. Yellow= tooth, blue= bone, red $=$ PDL 


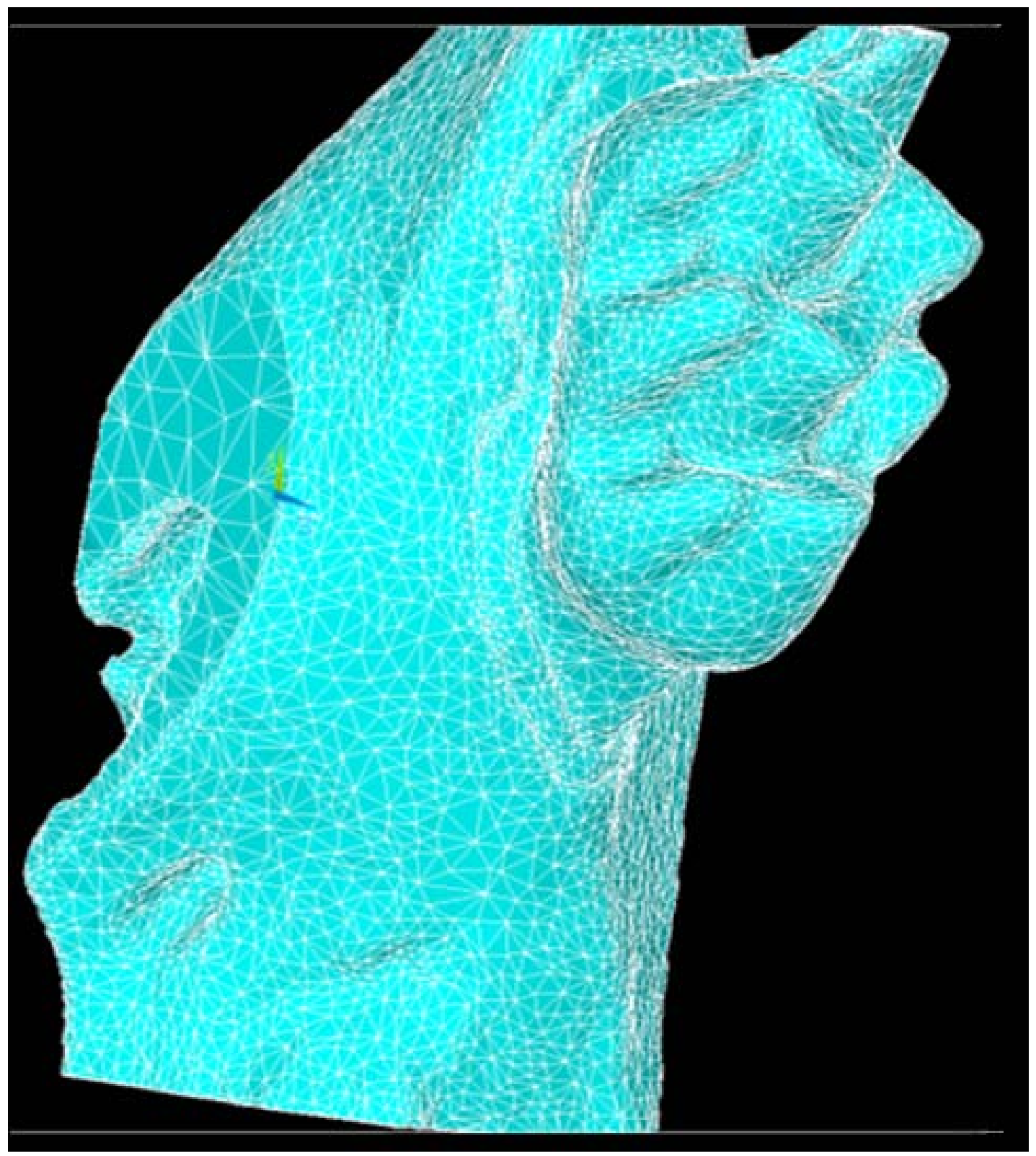

Figure 26. Resulting solid 3D finite element model obtained in ANSYS. 


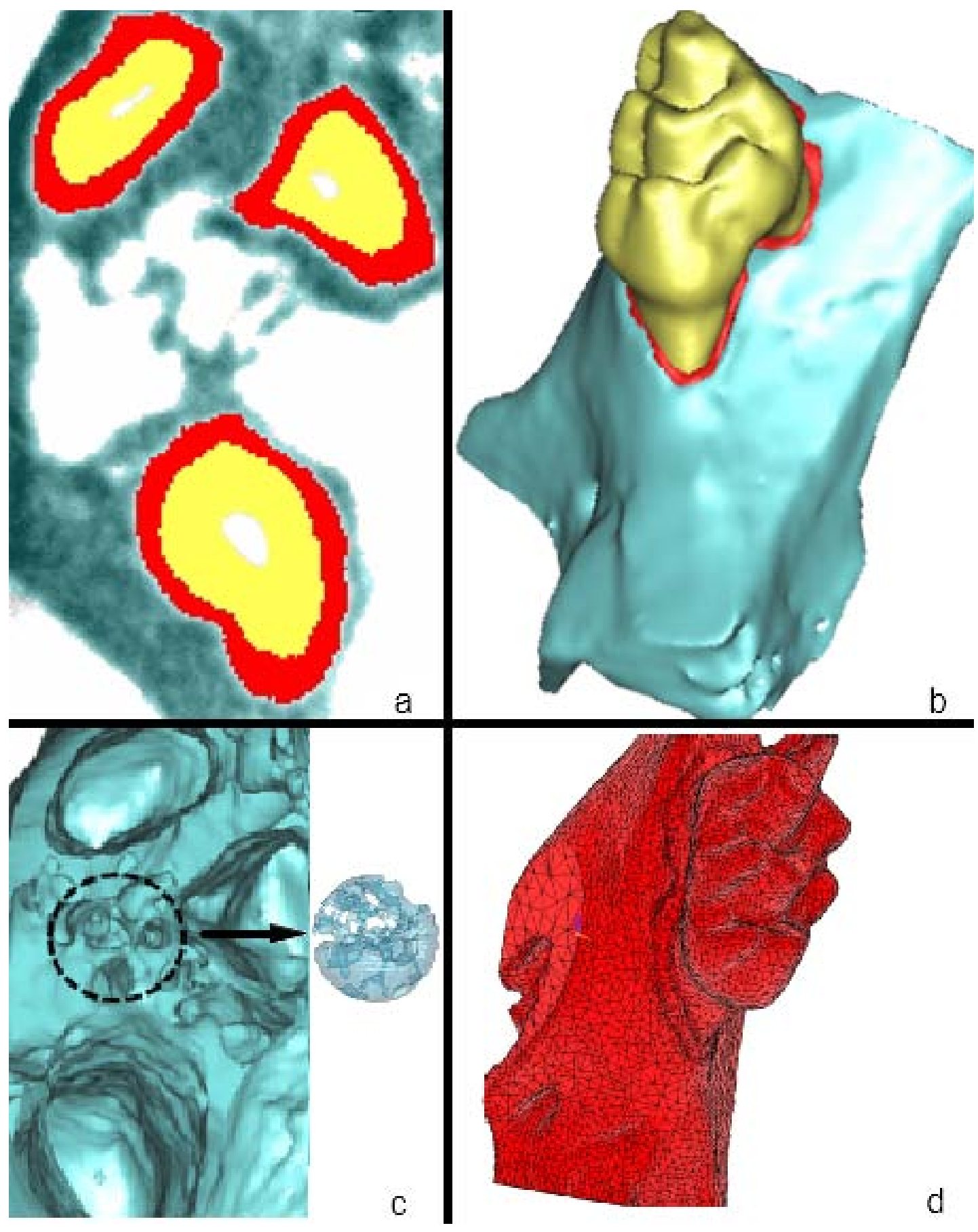

Figure 27. A, Segmentation and B, 3D reconstruction of the mouse molar. C, Isolated trabecular bone sphere for bone morphometry. D, Finite element model of the mouse molar. 


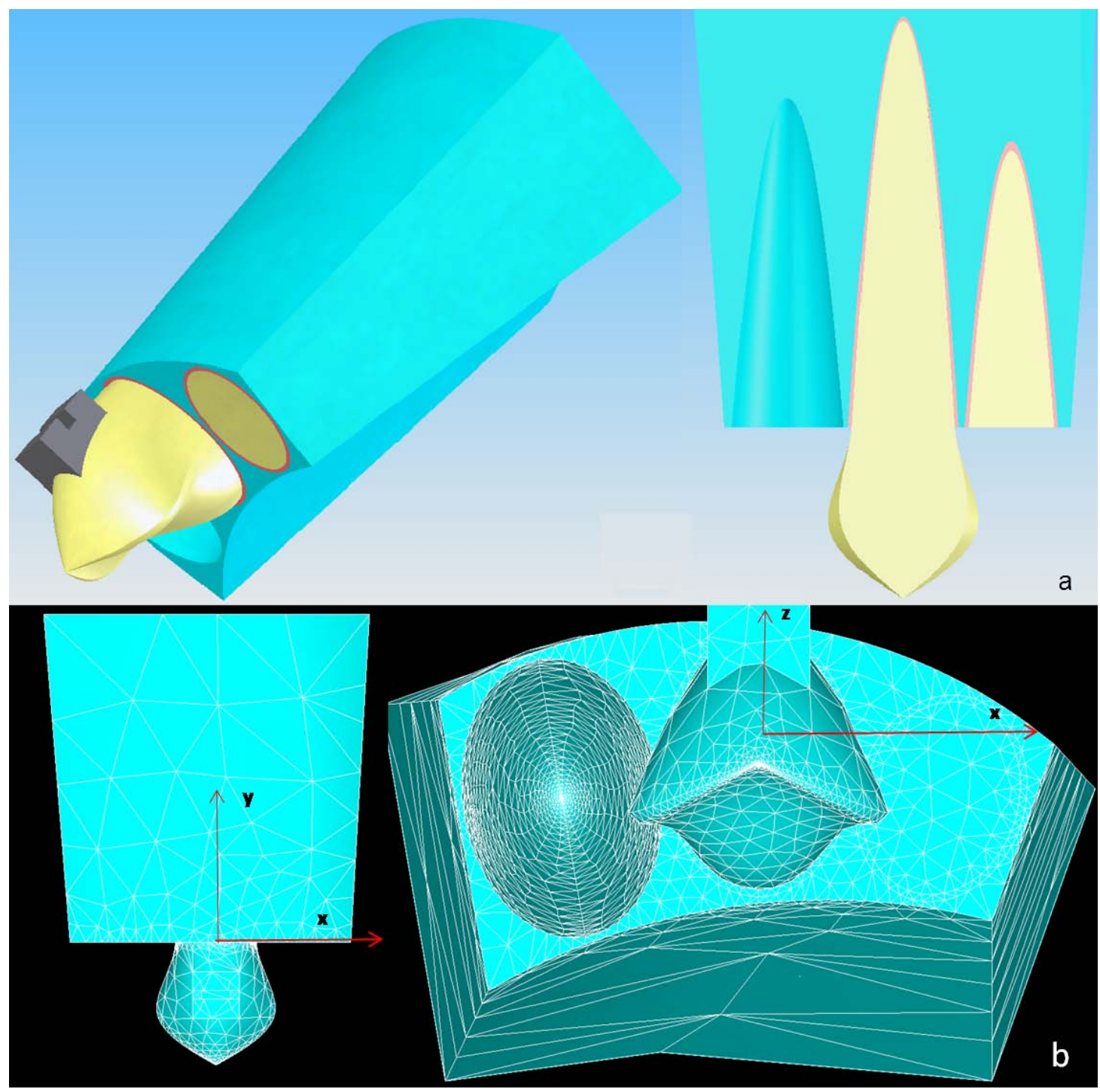

Figure 28. A, CAD model of the segment, and a cut showing the periodontal ligament, adjacent premolar socket and lateral incisor root. B, Finite element model. 


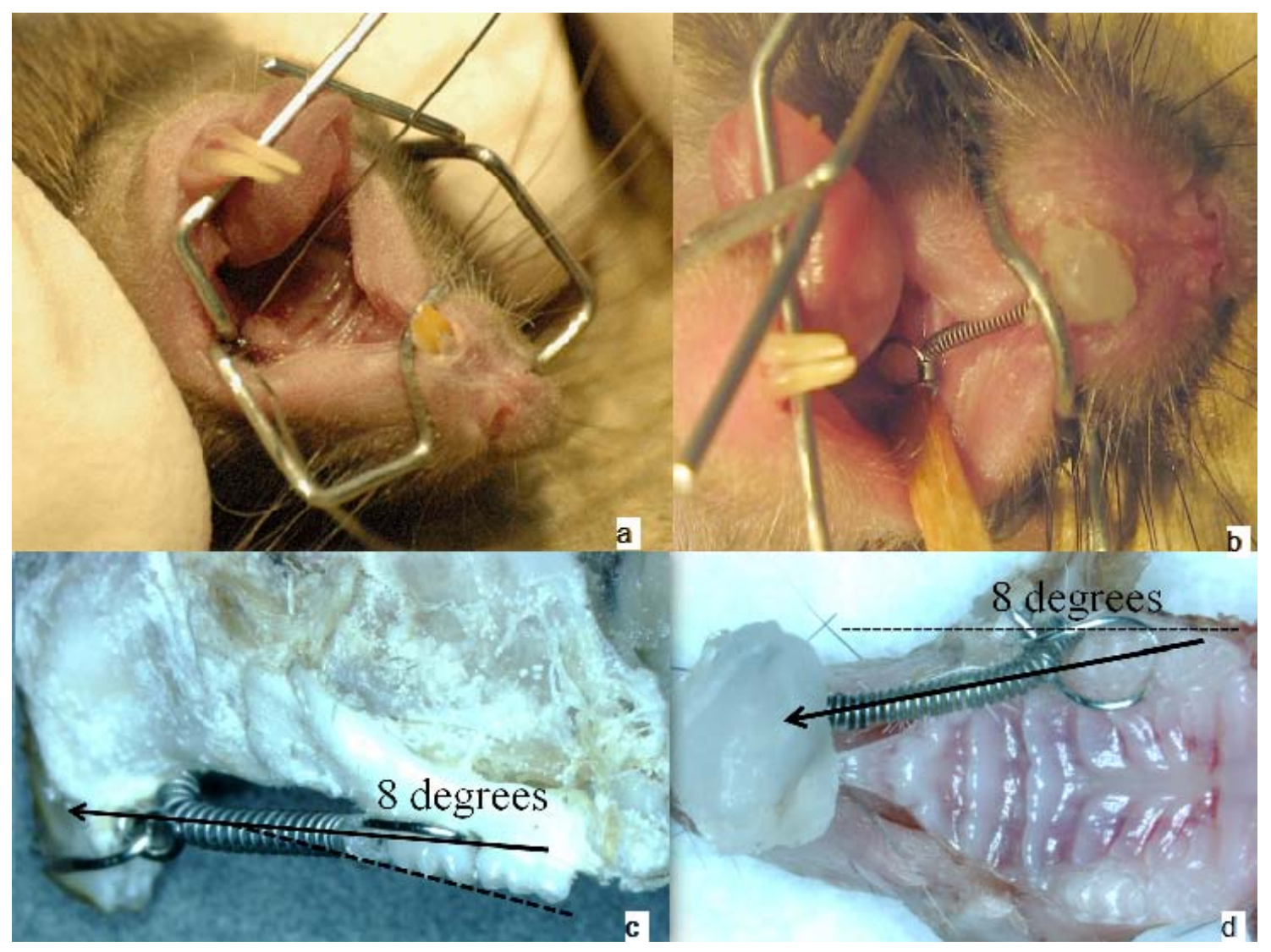

Figure 29. A, Mouse spring insertion procedure with custom prop. B, Spring in place. C, Sagittal and $\mathbf{D}$, occlusal views of the line of action of the force. 


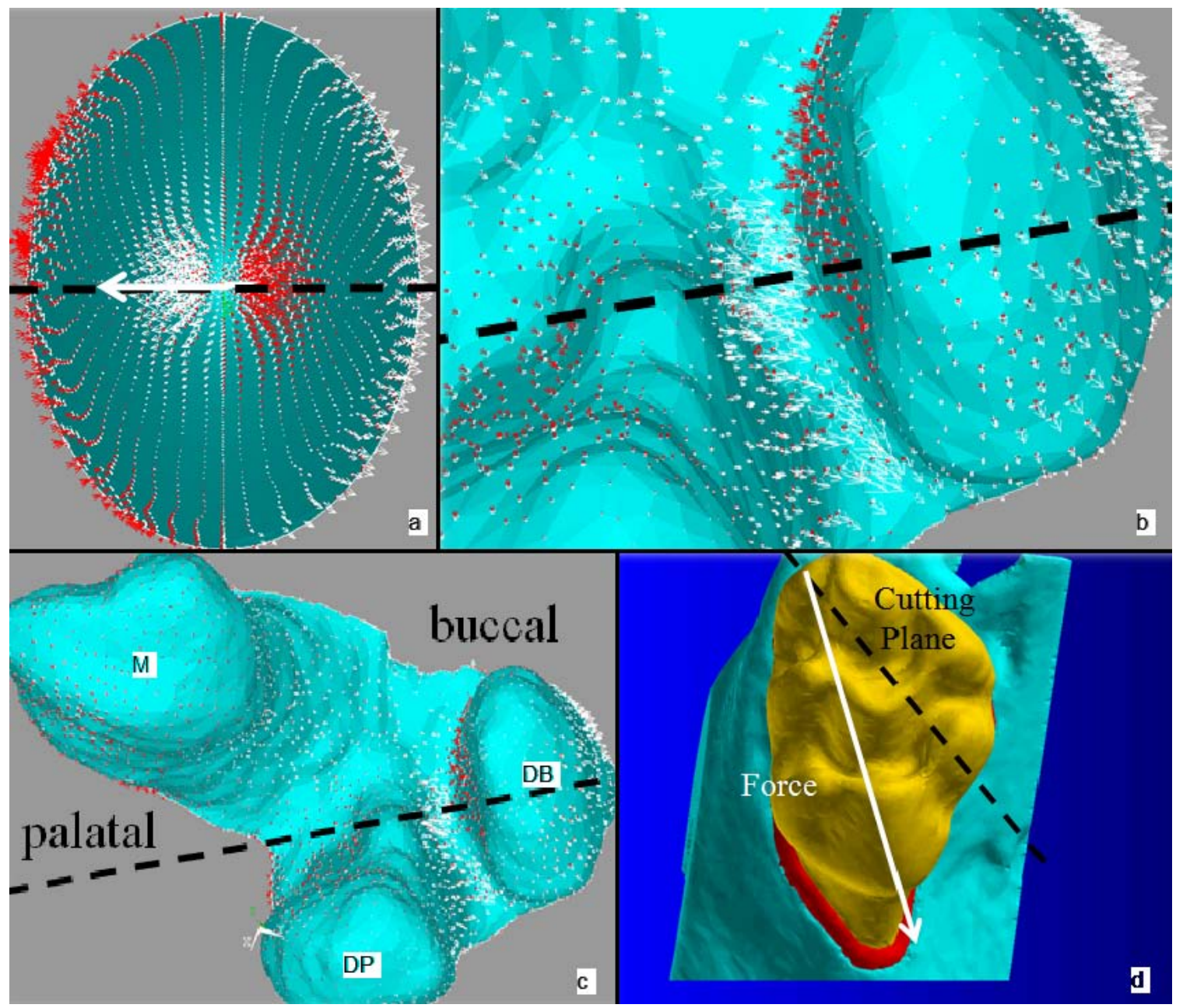

Figure 30. A, Occlusal view of idealized human canine PDL stress directions during tipping. The small red and white arrows represent maximum compressive and tensile stress directions, respectively. Note that the line of action of the force (large white arrow) and the maximum PDL stress directions are coincident with each other. B and $\mathbf{C}$ Apical views of the mouse PDL stress directions during tipping, with the correspondent root identification ( $\mathrm{DB}=$ disto-buccal, $\mathrm{DP}=$ disto-palatal and $\mathrm{M}=$ =mesial). Histological cutting plane for ERR analysis (dashed black line in $\mathbf{B}, \mathbf{C}$, and $\mathbf{D}$ ) follows the maximum compression PDL stress direction (red arrows), not the line of action of the force (white arrow in D.) 


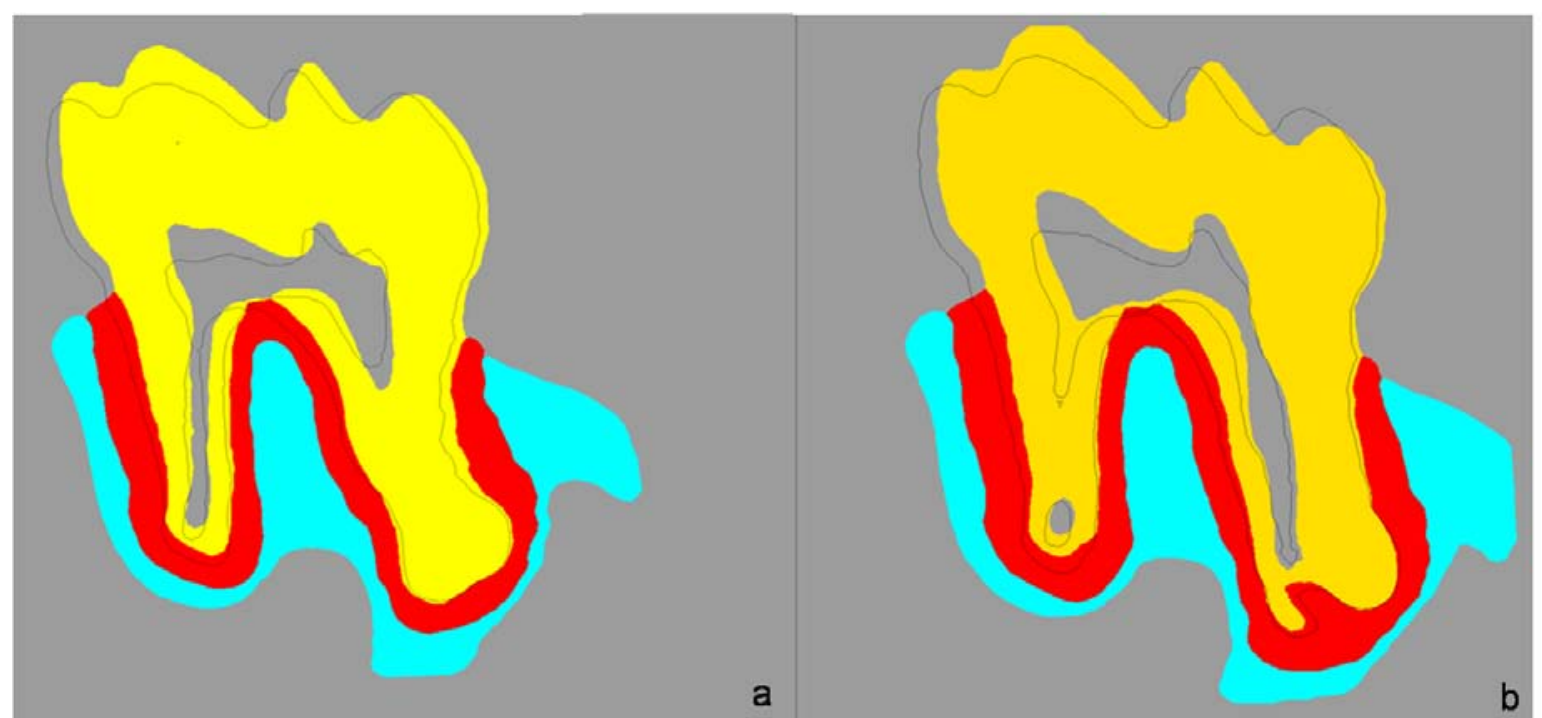

Figure 31. Static sections of the 3D FE model showing calculated initial (contour) and final (solid) positions of the teeth on the A, ERR analysis section plane and $\mathbf{B}$, bone formation analysis section plane. Because the sections followed stress directions, the tooth has negligible out-of- plane movement. This is evidenced by little change observed in tooth and root shape between the initial and final positions in these planes. 


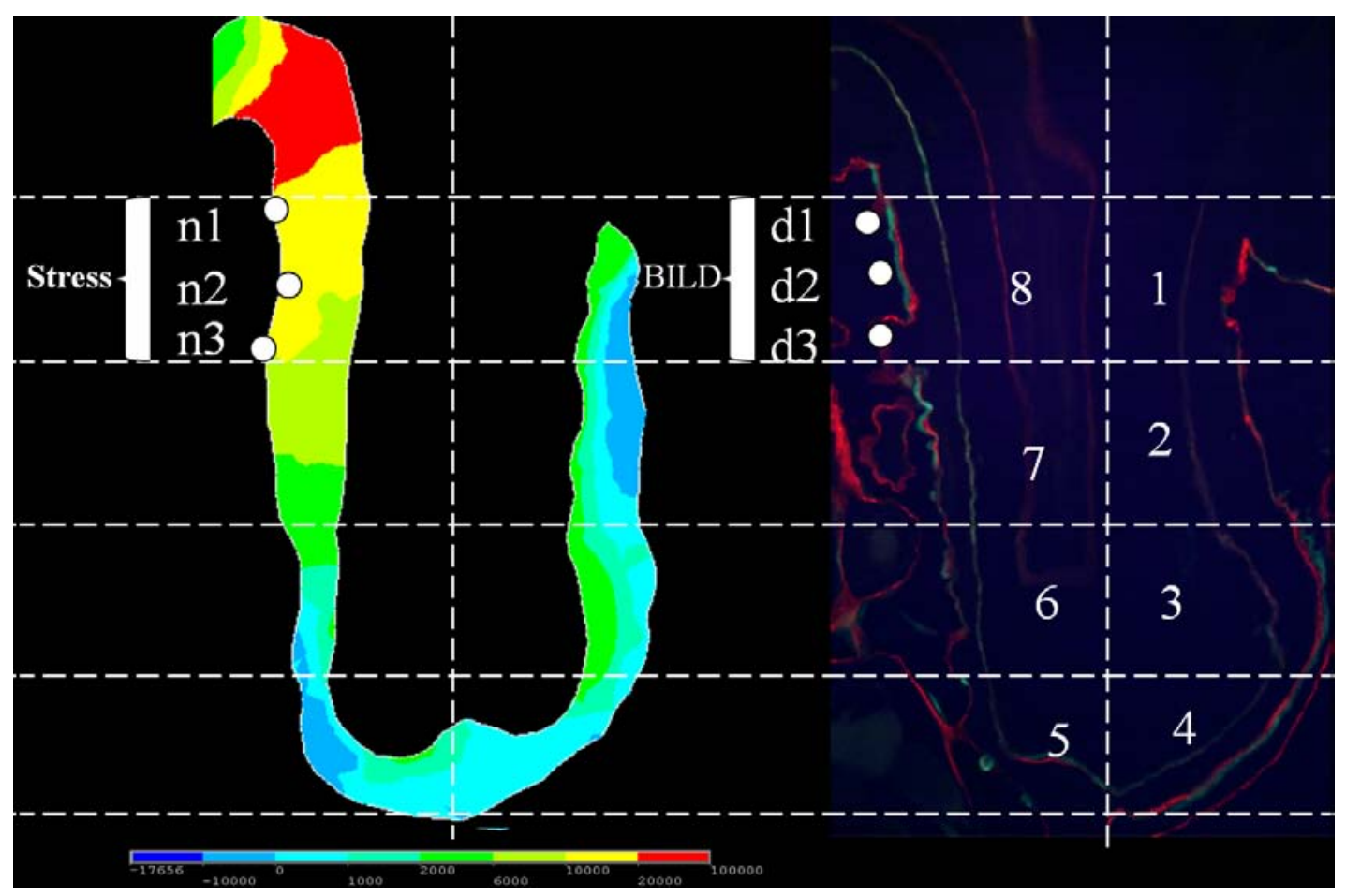

Figure 32. Method to relate FE-computed maximum PDL tensile stresses (left) to histologically measured interlabel bone distances (right.) In-plane maximum tensile stresses, acting horizontally, decrease from region 8 to 5 . Note that the pattern of PDL deformation was correctly predicted by the FEA. In bone measurements of the picture, $\mathrm{d} 3$ would be discarded because it is in a remodeling cavity, and only $\mathrm{d} 1$ and $\mathrm{d} 2$ would be averaged. The scale shows the maximum tensile stress values in Pascals. 


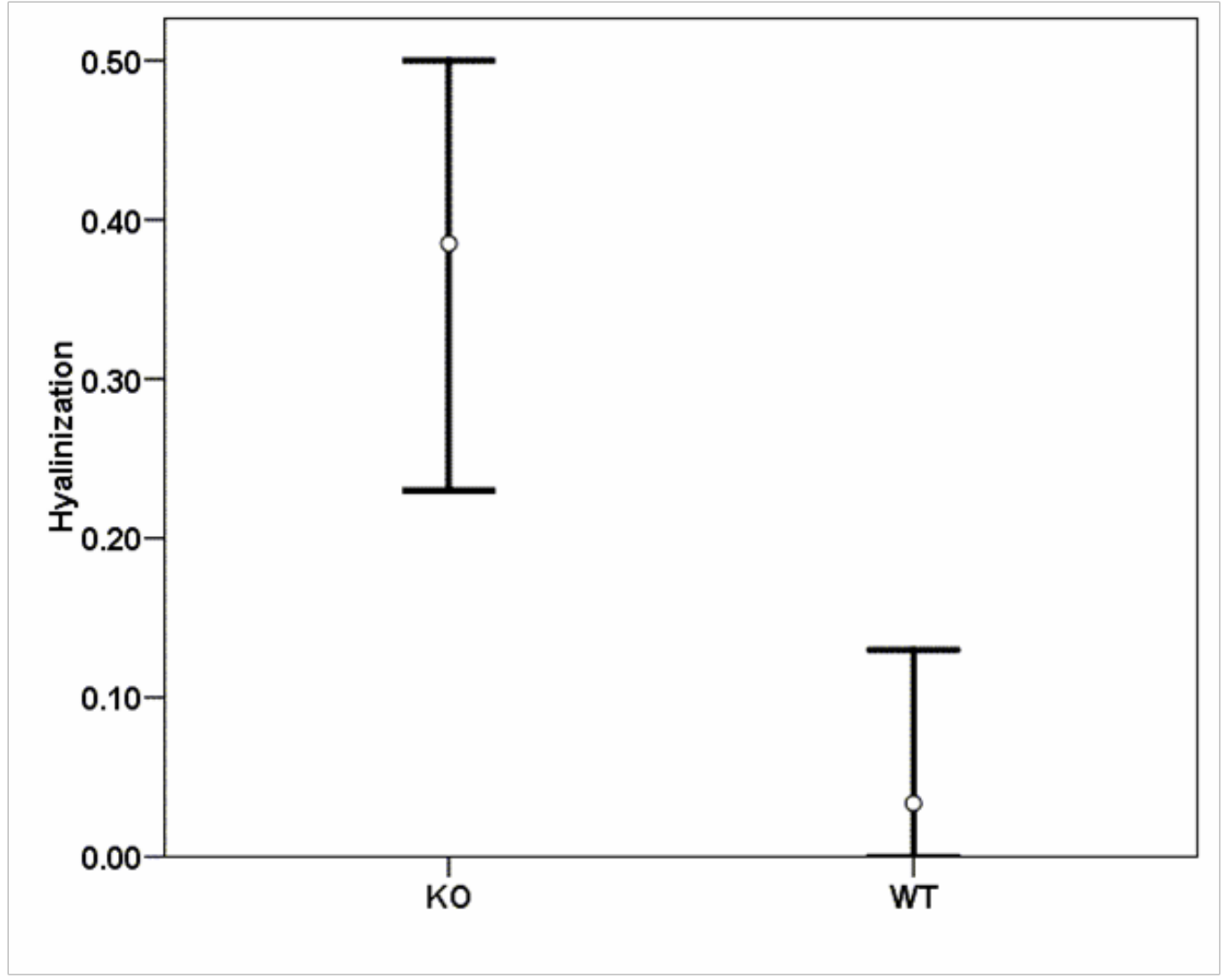

Figure 33. Comparison of tooth hyalinization scores in treated KO and WT mice $(\mathrm{p}<0.001)$. 


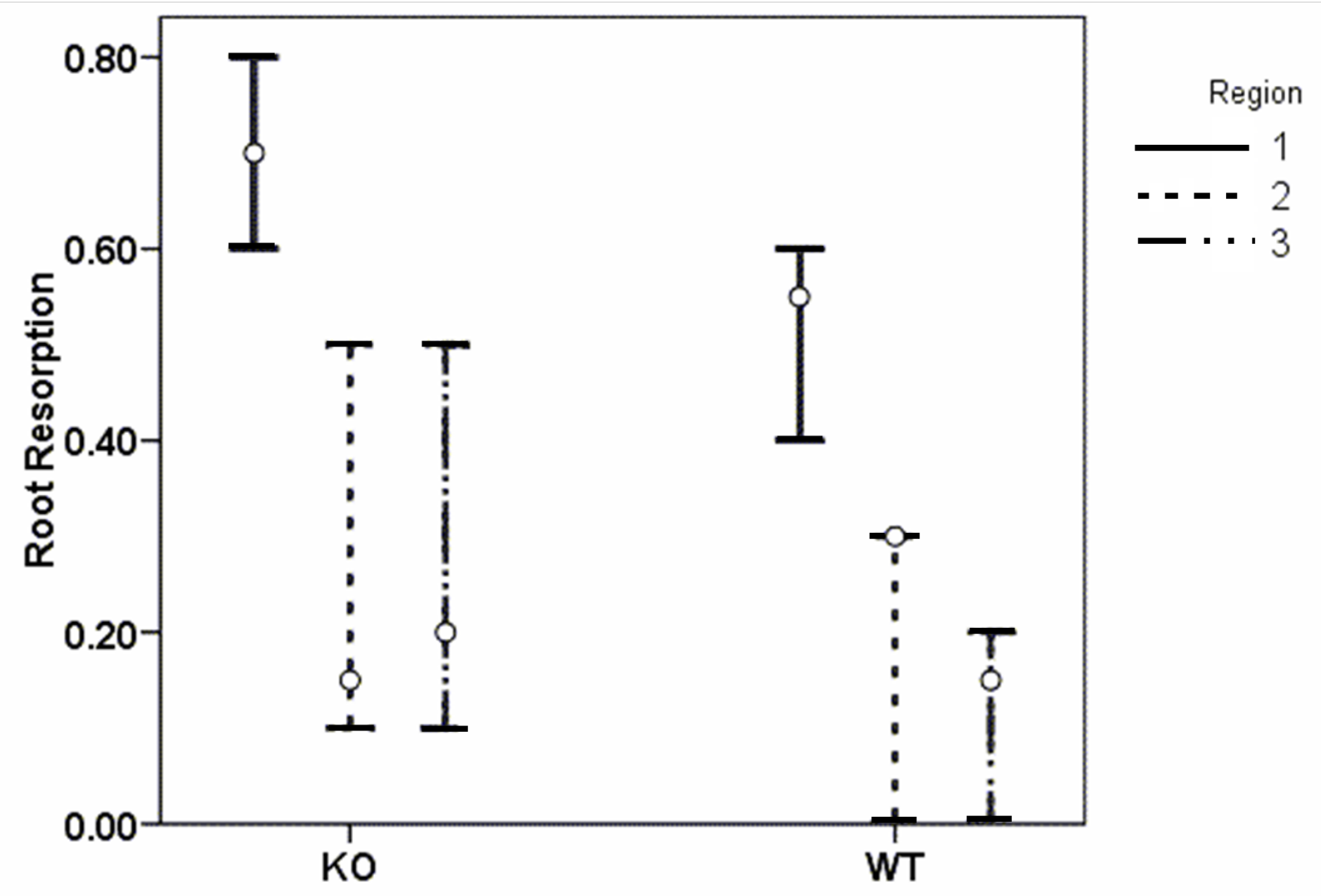

Figure 34. Comparison of ERR proportion score by region within each strain of treated mice. Significant differences in the WT and KO were found between regions 1 and 2 ( $\mathrm{p}=0.002, \mathrm{p}=0.001)$, and 1 and $3(\mathrm{p}<0.001, \mathrm{p}=0.002)$. 


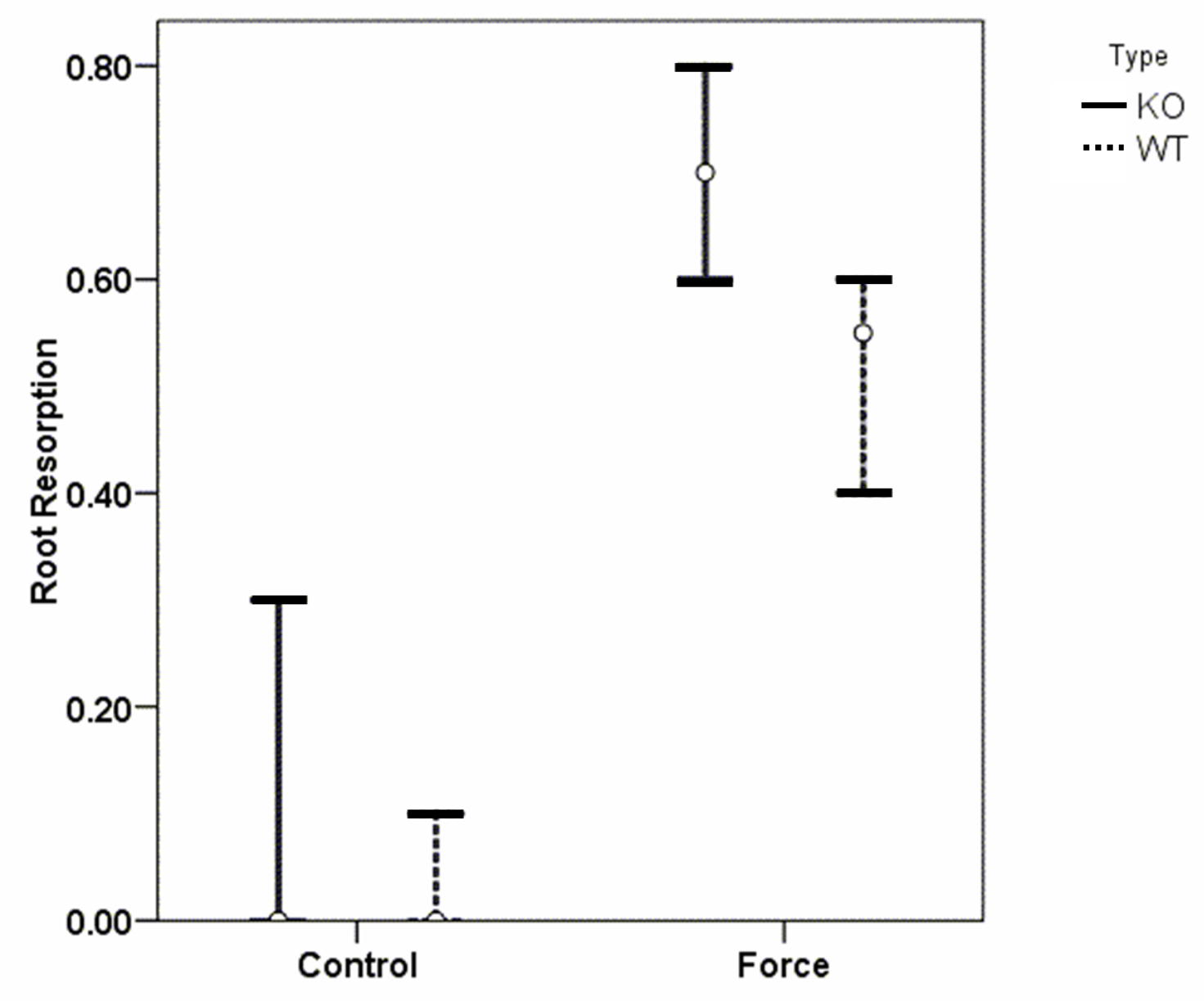

Figure 35. Comparison of ERR proportion score by type in the region of high stress $(\mathrm{p}<0.02)$. 


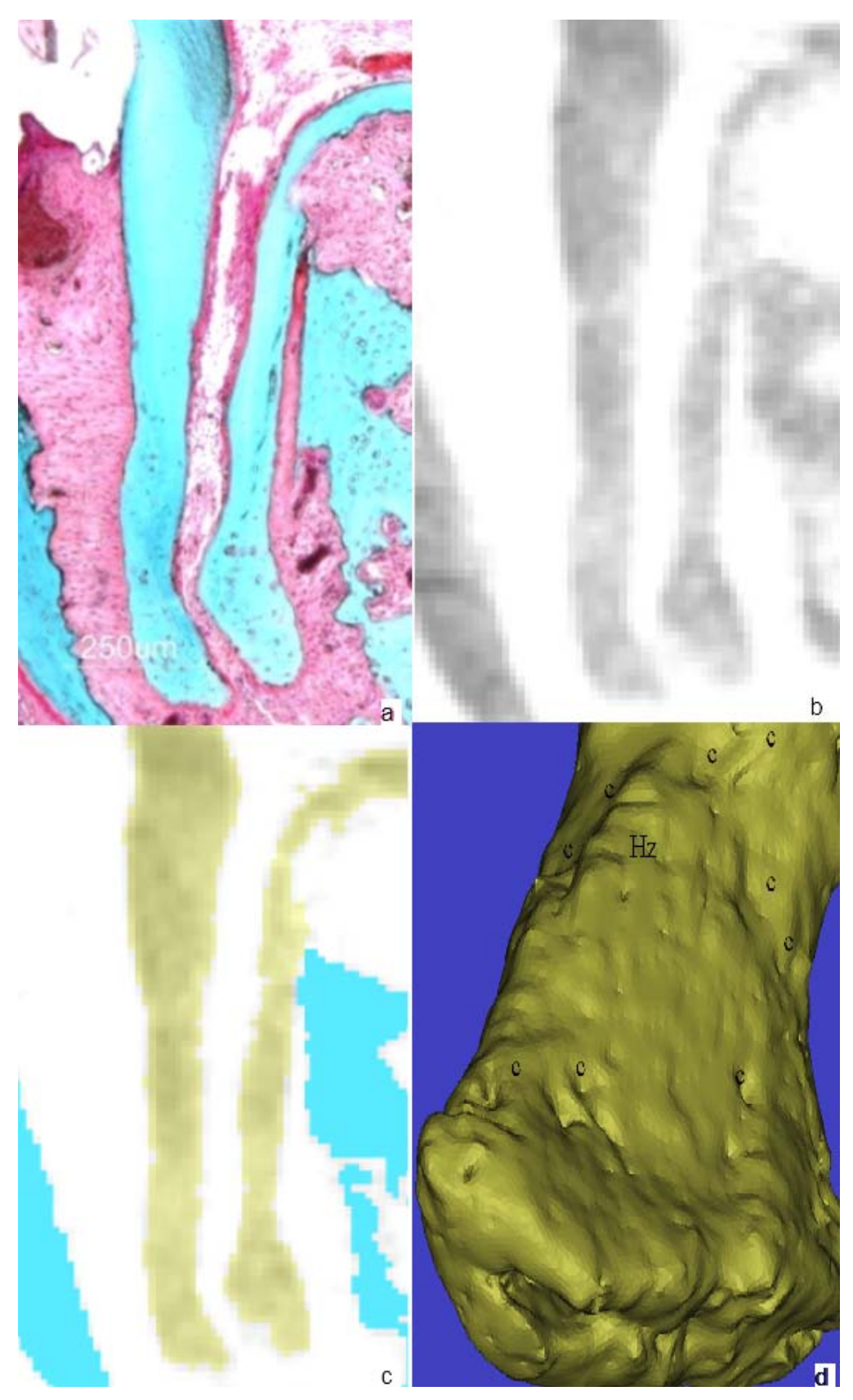

Figure 36. Illustration of microCT root resorption detection. A, Histological section of the DB root of a KO force group mouse. B, MicroCT reconstructed section of the same region and $\mathbf{C}$, its segmentation. Such segmentation of all sections allows 3D rendering of the structures and volume estimation. D, Visualization of resorption cavities (c) and the area of hyalinized PDL tissue (Hz). 


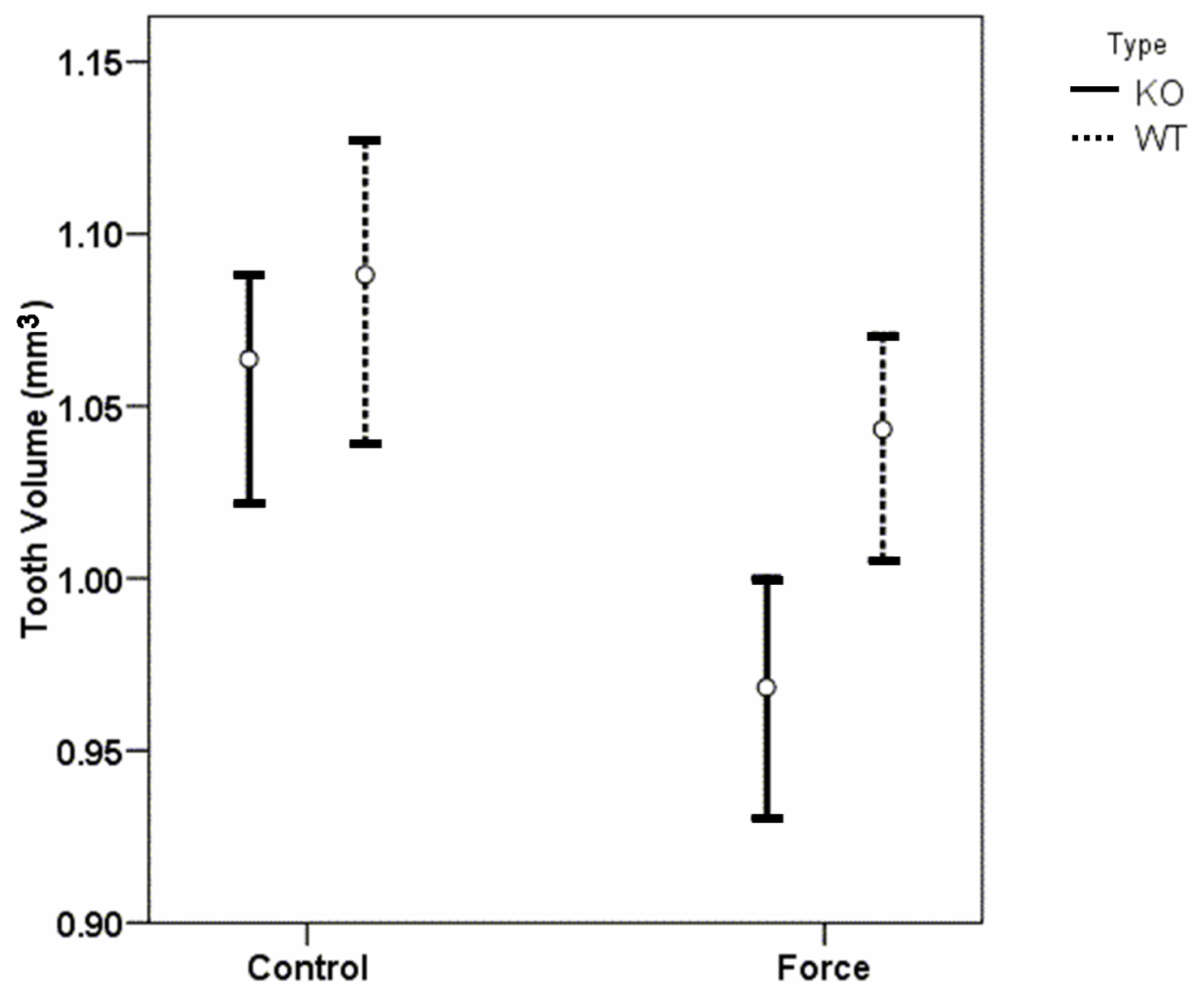

Figure 37. Tooth volume by type in control and force groups. A significant difference was found only after force application, confirming that KO has more ERR ( $p=0.002)$. 


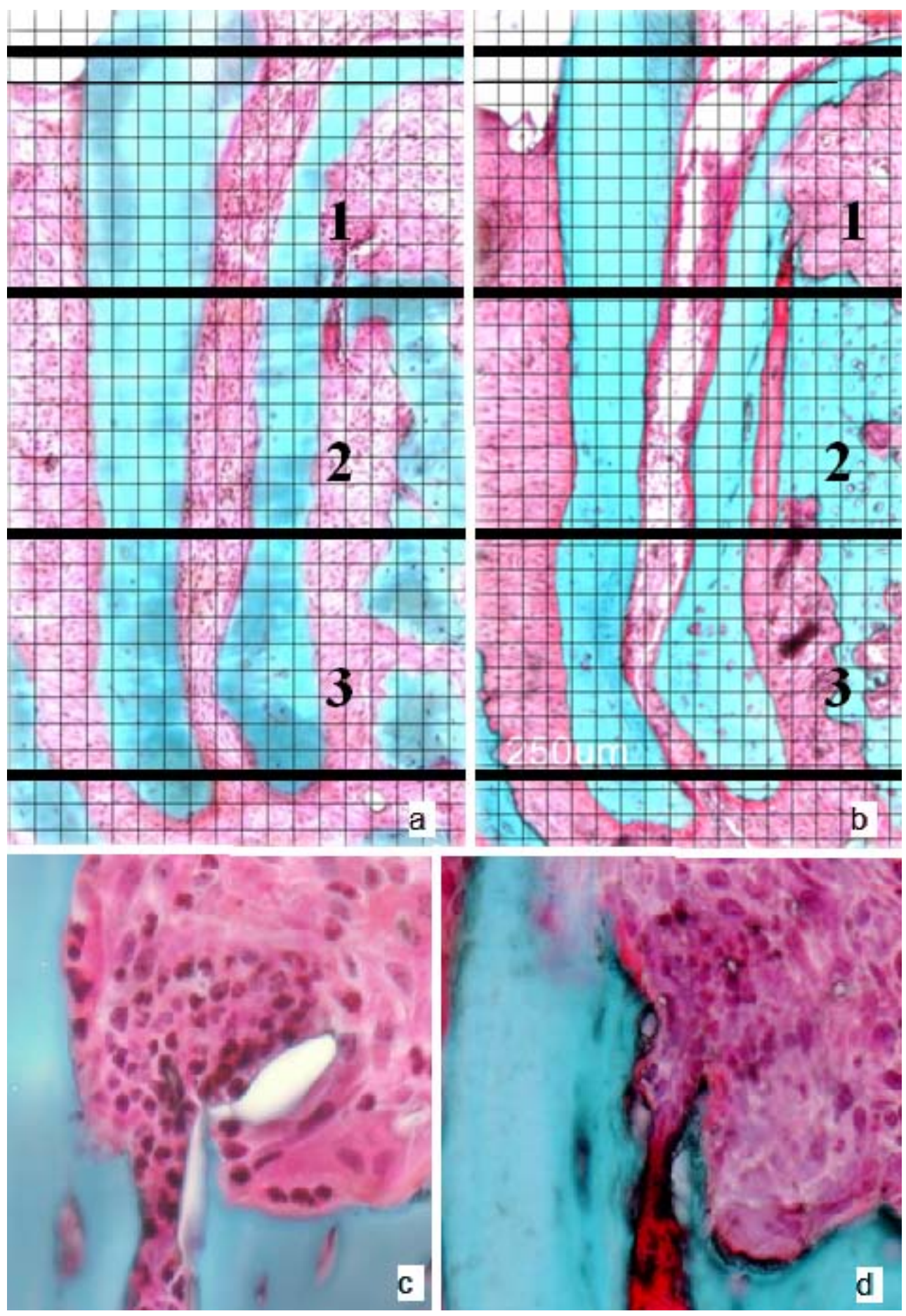

Figure 38. Example sections of WT (A and $\mathbf{C}$ ) and KO (B and $\mathbf{D})$ histology. $\mathbf{C}$ and $\mathbf{D}$ are magnified high stress regions of a and b, respectively. Note the increased presence of neutrophils (lobular shaped nuclei) in the WT section. 


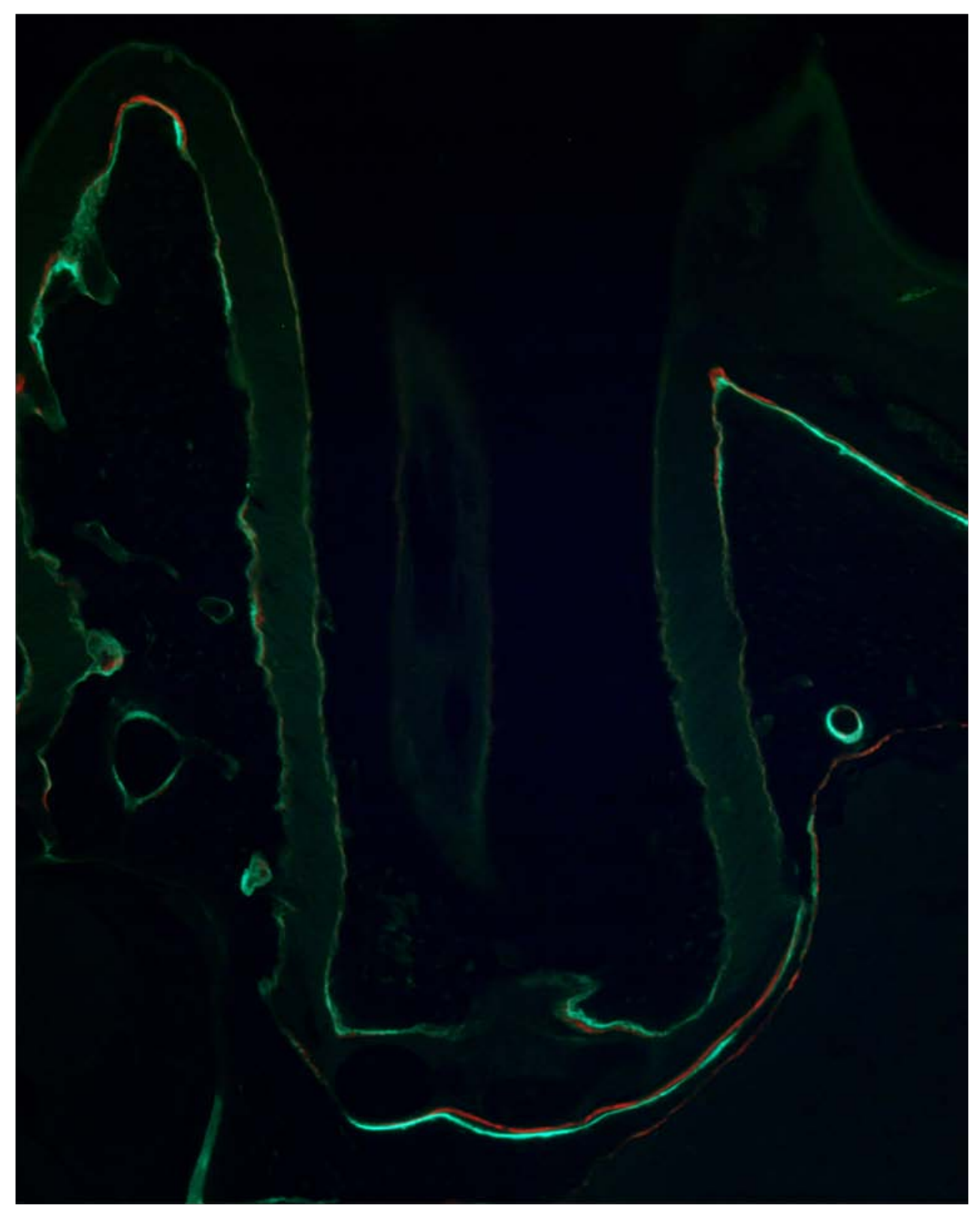

Figure 39. Example section of a labeled control WT disto-palatal root. Note normal bone apposition around blood vessels. New bone is also observed on the palatal periosteal surface and near the apex, suggesting slight eruption during the experimental period. Due to this interference, the apical regions (2 apical region 5 points and region 6 ) were eliminated from linear regression analysis in the treated mice. 


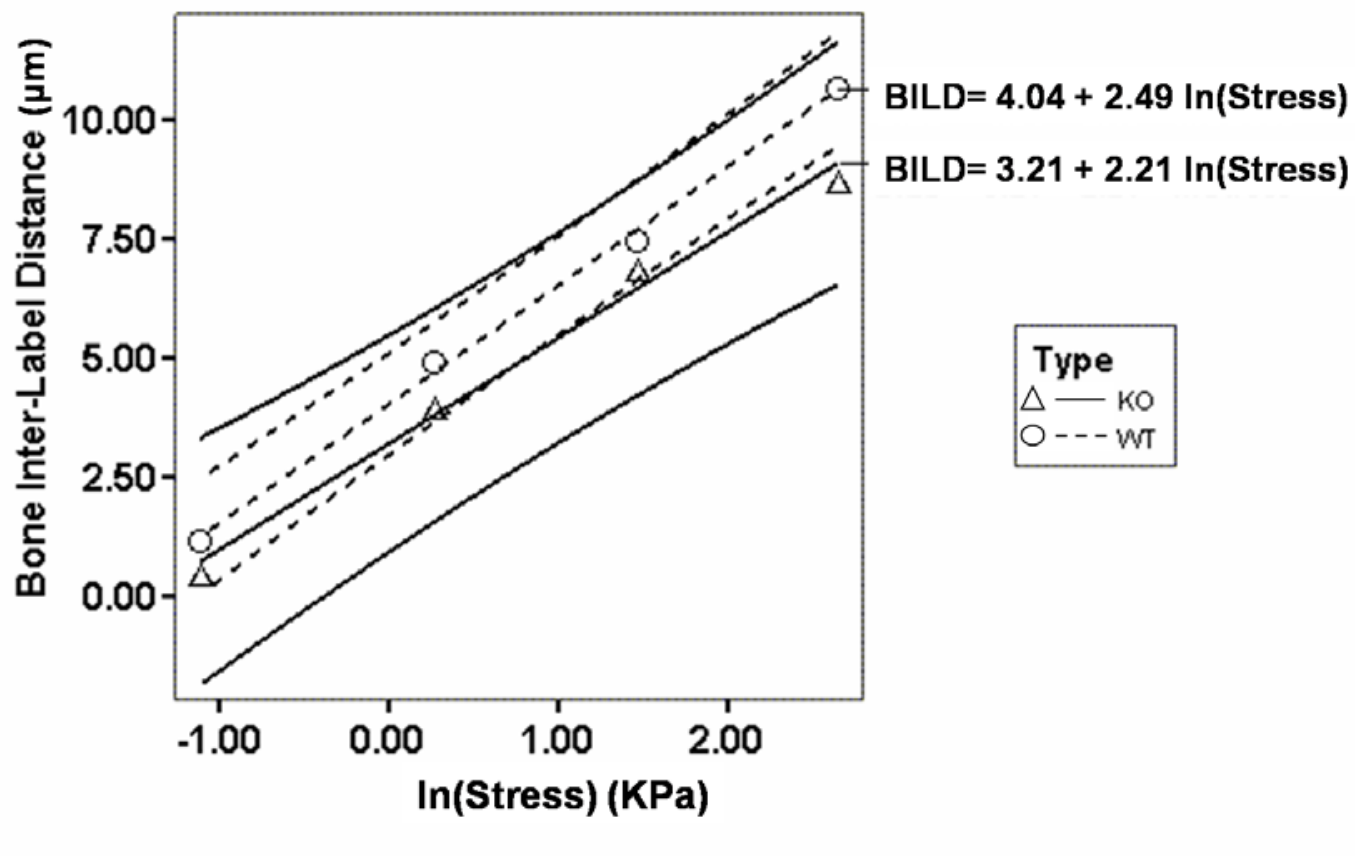

Figure 40. Linear regression plots of the natural logarithm of maximum principal stress [ln (Stress)] vs. Bone Interlabel Distance in the DP root of WT and KO mice. The outer lines in each regression are the limits of the 95\% confidence interval. 


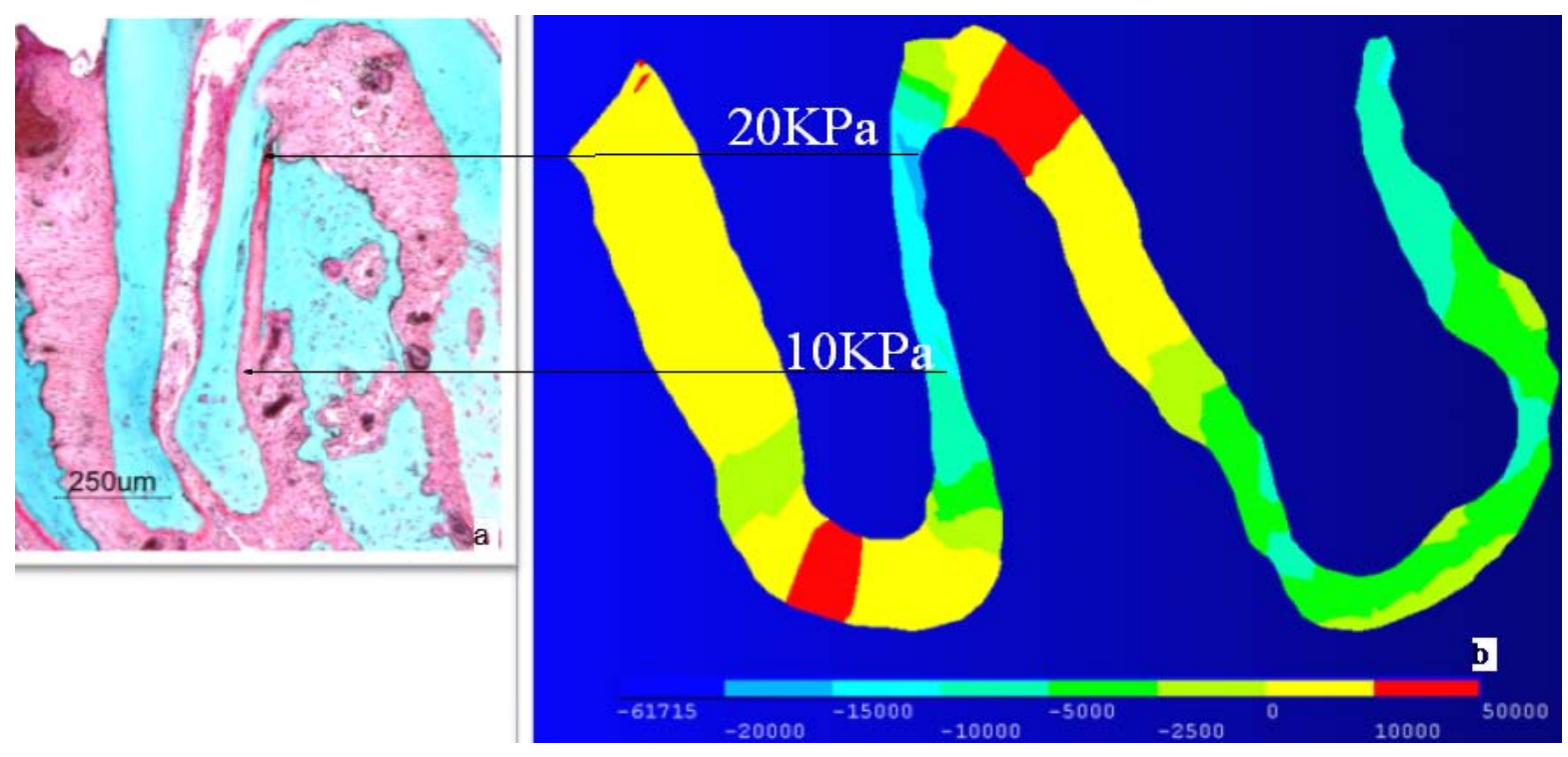

Figure 41. A, Histological section of a KO mouse. Large area of hyalinized tissue remains on the compression side of the DB root. $\mathbf{B}$, Corresponding section of the FE model. Note that the FEA correctly predicted the deformation of the PDL. Hyalinization occurs at PDL stress levels $>\sim 10 \mathrm{KPa}$. 


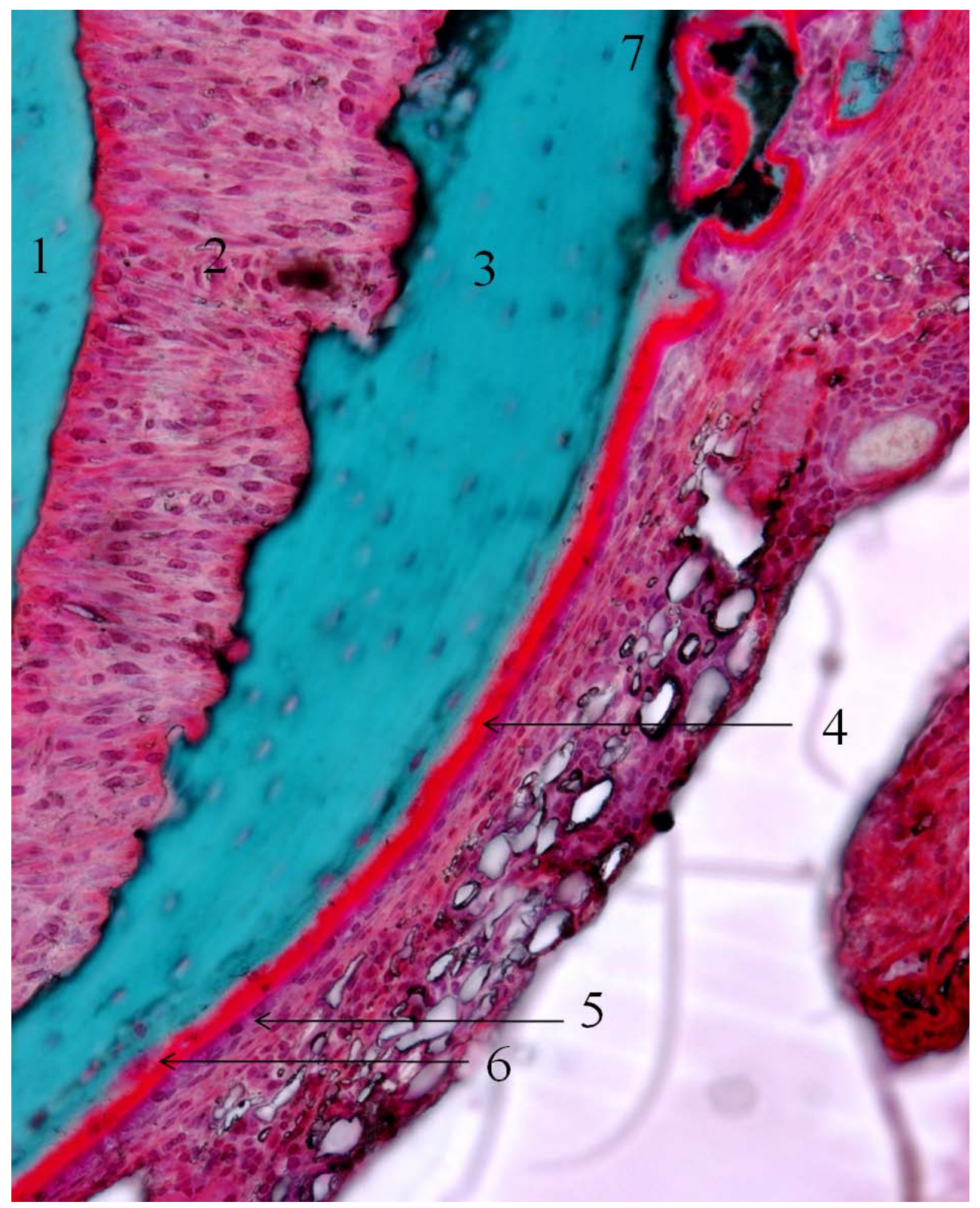

Figure 42. Structures around the high-tension PDL of DB root. Note stretched fibroblasts and the absence of bone formation and inflammatory cells within the socket. Bone formation, however, is prominent on the periosteum of the buccal surface. (1) Root. (2) PDL with stretched fibroblasts. (3) Buccal alveolar wall. (4) Osteoid. (5) Osteoblasts. (6) Trapped osteoblast that would become an osteocyte. (7) Cervical area where once there was resorption, and now there is formation. 


\section{REFERENCES}

Agarwal, S., Chandra, C. S., Piesco, N. P., Langkamp, H. H., Bowen, L. \& Baran, C. (1998). Regulation of periodontal ligament cell functions by interleukin-1beta. Infect Immun 66, 932-7.

Agarwal, S., Long, P., Seyedain, A., Piesco, N., Shree, A. \& Gassner, R. (2003). A central role for the nuclear factor-kappaB pathway in anti-inflammatory and proinflammatory actions of mechanical strain. Faseb J 17, 899-901.

Akin, E., Gurton, A. U. \& Olmez, H. (2004). Effects of nitric oxide in orthodontic tooth movement in rats. Am J Orthod Dentofacial Orthop 126, 608-14.

Al-Qawasmi, R. A., Hartsfield, J. K., Hartsfield, J. K., Jr., Everett, E. T., Weaver, M. R., Foroud, T. M. \& Roberts, W. E. (2004). Root resorption associated with orthodontic force in IL-1Beta knockout mouse. J Musculoskelet Neuronal Interact 4, 383-5.

Al-Qawasmi, R. A., Hartsfield, J. K., Jr., Everett, E. T., Flury, L., Liu, L., Foroud, T. M., Macri, J. V. \& Roberts, W. E. (2003a). Genetic predisposition to external apical root resorption. Am J Orthod Dentofacial Orthop 123, 242-52.

Al-Qawasmi, R. A., Hartsfield, J. K., Jr., Everett, E. T., Flury, L., Liu, L., Foroud, T. M., Macri, J. V. \& Roberts, W. E. (2003b). Genetic predisposition to external apical root resorption in orthodontic patients: linkage of chromosome-18 marker. J Dent Res 82, 356-60.

Al-Qawasmi, R. A., Hartsfield, J. K., Jr., Everett, E. T., Weaver, M. R., Foroud, T. M., Faust, D. M. \& Roberts, W. E. (2006). Root resorption associated with orthodontic force in inbred mice: genetic contributions. Eur J Orthod 28, 13-9.

Andersen, K. L., Mortensen, H. T., Pedersen, E. H. \& Melsen, B. (1991a). Determination of stress levels and profiles in the periodontal ligament by means of an improved three-dimensional finite element model for various types of orthodontic and natural force systems. J Biomed Eng 13, 293-303.

Andersen, K. L., Pedersen, E. H. \& Melsen, B. (1991b). Material parameters and stress profiles within the periodontal ligament. Am J Orthod Dentofacial Orthop 99, 427-40.

Bao, B., Zhao, M. \& Rao, Y. (1995). [The effects of rat condylar endogenous prostaglandin E2 on the mandiblar growth and adaptation during the rat mandible functional protraction]. Zhonghua Kou Qiang Yi Xue Za Zhi 30, 365-6.

Barlow, P. G., Li, Y., Wilkinson, T. S., Bowdish, D. M., Lau, Y. E., Cosseau, C., Haslett, C., Simpson, A. J., Hancock, R. E. \& Davidson, D. J. (2006). The human cationic host defense peptide LL-37 mediates contrasting effects on apoptotic pathways in different primary cells of the innate immune system. J Leukoc Biol 80, 509-20.

Bassett, C. A. \& Becker, R. O. (1962). Generation of electric potentials by bone in response to mechanical stress. Science 137, 1063-4.

Bassett, C. A., Pawluk, R. J. \& Becker, R. O. (1964). Effects of Electric Currents on Bone in Vivo. Nature 204, 652-4.

Baumrind, S. (1969). A reconsideration of the propriety of the "pressure-tension" hypothesis. Am J Orthod 55, 12-22. 
Baylink, D. J., Finkelman, R. D. \& Mohan, S. (1993). Growth factors to stimulate bone formation. J Bone Miner Res 8 Suppl 2, S565-72.

Beer, F. J. J., ER; Dewolf JT. (2002). Mechanics of Materials, 3rd edition. Mcgraw-Hill, New York.

Bianchi, M.E. (2007). DAMPs, PAMPs and alarmins: all we need to know about danger. J Leukoc Biol 81, 1-5.

Bidwell, J. P., Yang, J., Robling, A. G. (2008). Is HMGB1 an osteocyte alarmin? J Cell Biochem. 103, 1671-80.

Bodin, P. \& Burnstock, G. (1998). Increased release of ATP from endothelial cells during acute inflammation. Inflamm Res 47, 351-4.

Boekenoogen, D. I., Sinha, P. K., Nanda, R. S., Ghosh, J., Currier, G. F. \& Howes, R. I. (1996). The effects of exogenous prostaglandin E2 on root resorption in rats. Am J Orthod Dentofacial Orthop 109, 277-86.

Bonewald, L. F. \& Mundy, G. R. (1990). Role of transforming growth factor-beta in bone remodeling. Clin Orthop Relat Res, 261-76.

Brady, T. A., Piesco, N. P., Buckley, M. J., Langkamp, H. H., Bowen, L. L. \& Agarwal, S. (1998). Autoregulation of periodontal ligament cell phenotype and functions by transforming growth factor-beta1. J Dent Res 77, 1779-90.

Brezniak, N. \& Wasserstein, A. (1993a). Root resorption after orthodontic treatment: Part 1. Literature review. Am J Orthod Dentofacial Orthop 103, 62-6.

Brezniak, N. \& Wasserstein, A. (1993b). Root resorption after orthodontic treatment: Part 2. Literature review. Am J Orthod Dentofacial Orthop 103, 138-46.

Brezniak, N. \& Wasserstein, A. (2002a). Orthodontically induced inflammatory root resorption. Part I: The basic science aspects. Angle Orthod 72, 175-9.

Brezniak, N. \& Wasserstein, A. (2002b). Orthodontically induced inflammatory root resorption. Part II: The clinical aspects. Angle Orthod 72, 180-4.

Brough, D., Le Feuvre, R. A., Wheeler, R. D., Solovyova, N., Hilfiker, S., Rothwell, N. J. \& Verkhratsky, A. (2003). Ca2+ stores and Ca2+ entry differentially contribute to the release of IL-1 beta and IL-1 alpha from murine macrophages. $J$ Immunol 170, 3029-36.

Brudvik, P. \& Rygh, P. (1991). Root resorption after local injection of prostaglandin E2 during experimental tooth movement. Eur J Orthod 13, 255-63.

Brudvik, P. \& Rygh, P. (1993a). The initial phase of orthodontic root resorption incident to local compression of the periodontal ligament. Eur J Orthod 15, 249-63.

Brudvik, P. \& Rygh, P. (1993b). Non-clast cells start orthodontic root resorption in the periphery of hyalinized zones. Eur J Orthod 15, 467-80.

Brudvik, P. \& Rygh, P. (1994a). Multi-nucleated cells remove the main hyalinized tissue and start resorption of adjacent root surfaces. Eur J Orthod 16, 265-73.

Brudvik, P. \& Rygh, P. (1994b). Root resorption beneath the main hyalinized zone. Eur $J$ Orthod 16, 249-63.

Brudvik, P. \& Rygh, P. (1995a). The repair of orthodontic root resorption: an ultrastructural study. Eur J Orthod 17, 189-98.

Brudvik, P. \& Rygh, P. (1995b). Transition and determinants of orthodontic root resorption-repair sequence. Eur J Orthod 17, 177-88.

Burnstock, G. (1999). Release of vasoactive substances from endothelial cells by shear stress and purinergic mechanosensory transduction. J Anat 194 ( Pt 3), 335-42. 
Burr, D. B., Robling, A. G. \& Turner, C. H. (2002). Effects of biomechanical stress on bones in animals. Bone 30, 781-6.

Burstone, C. J. \& Pryputniewicz, R. J. (1980). Holographic determination of centers of rotation produced by orthodontic forces. Am J Orthod 77, 396-409.

Canalis, E., Centrella, M., Burch, W. \& McCarthy, T. L. (1989). Insulin-like growth factor I mediates selective anabolic effects of parathyroid hormone in bone cultures. J Clin Invest 83, 60-5.

Cattaneo, P. M., Dalstra, M. \& Melsen, B. (2005). The finite element method: a tool to study orthodontic tooth movement. J Dent Res 84, 428-33.

Chakfe, Y., Seguin, R., Antel, J. P., Morissette, C., Malo, D., Henderson, D. \& Seguela, P. (2002). ADP and AMP induce interleukin-1beta release from microglial cells through activation of ATP-primed P2X7 receptor channels. J Neurosci 22, 30619.

Chan, E. \& Darendeliler, M. A. (2005). Physical properties of root cementum: Part 5. Volumetric analysis of root resorption craters after application of light and heavy orthodontic forces. Am J Orthod Dentofacial Orthop 127, 186-95.

Chan, E. \& Darendeliler, M. A. (2006). Physical properties of root cementum: part 7. Extent of root resorption under areas of compression and tension. Am J Orthod Dentofacial Orthop 129, 504-10.

Chan, E. K. \& Darendeliler, M. A. (2004). Exploring the third dimension in root resorption. Orthod Craniofac Res 7, 64-70.

Chan, E. K., Darendeliler, M. A., Jones, A. S. \& Kaplin, I. J. (2004a). A calibration method used for volumetric measurement of orthodontically induced root resorption craters. Ann Biomed Eng 32, 880-8.

Chan, E. K., Darendeliler, M. A., Petocz, P. \& Jones, A. S. (2004b). A new method for volumetric measurement of orthodontically induced root resorption craters. Eur $J$ Oral Sci 112, 134-9.

Chan, E. K., Petocz, P. \& Darendeliler, M. A. (2005). Validation of two-dimensional measurements of root resorption craters on human premolars after 28 days of force application. Eur J Orthod 27, 390-5.

Chao, C. F., Shih, C., Wang, T. M. \& Lo, T. H. (1988). Effects of prostaglandin E2 on alveolar bone resorption during orthodontic tooth movement. Acta Anat (Basel) 132, 304-9.

Chen, J., Bulucea, I., Katona, T. R. \& Ofner, S. (2007). Complete orthodontic load systems on teeth in a continuous full archwire: the role of triangular loop position. Am J Orthod Dentofacial Orthop 132, 143 e1-8.

Chen, L. \& Brosnan, C. F. (2006). Regulation of Immune Response by P2X7 Receptor. Crit Rev Immunol 26, 499-513.

Chen, W. F. \& Lui, E. M. (2005). Handbook of structural engineering., 2nd edition. CRC Press, London, UK.

Choy, K., Pae, E. K., Park, Y., Kim, K. H. \& Burstone, C. J. (2000). Effect of root and bone morphology on the stress distribution in the periodontal ligament. Am J Orthod Dentofacial Orthop 117, 98-105.

Christiansen, R. L. \& Burstone, C. J. (1969). Centers of rotation within the periodontal space. Am J Orthod 55, 353-69. 
Collo, G., Neidhart, S., Kawashima, E., Kosco-Vilbois, M., North, R. A. \& Buell, G. (1997). Tissue distribution of the P2X7 receptor. Neuropharmacology 36, 127783.

Copeland, S. \& Green, L. J. (1986). Root resorption in maxillary central incisors following active orthodontic treatment. Am J Orthod 89, 51-5.

Darendeliler, M. A., Kharbanda, O. P., Chan, E. K., Srivicharnkul, P., Rex, T., Swain, M. V., Jones, A. S. \& Petocz, P. (2004). Root resorption and its association with alterations in physical properties, mineral contents and resorption craters in human premolars following application of light and heavy controlled orthodontic forces. Orthod Craniofac Res 7, 79-97.

Davidovitch, Z. (1991). Tooth movement. Crit Rev Oral Biol Med 2, 411-50.

Davidovitch, Z., Nicolay, O. F., Ngan, P. W. \& Shanfeld, J. L. (1988). Neurotransmitters, cytokines, and the control of alveolar bone remodeling in orthodontics. Dent Clin North Am 32, 411-35.

Dorow, C. \& Sander, F. G. (2005). Development of a Model for the Simulation of Orthodontic Load on Lower First Premolars Using the Finite Element Method. $J$ Orofac Orthop 66, 208-218.

Epker, B. N. \& Frost, H. M. (1965). Correlation of Bone Resorption and Formation with the Physical Behavior of Loaded Bone. J Dent Res 44, 33-41.

Ferrari, D., Pizzirani, C., Adinolfi, E., Lemoli, R. M., Curti, A., Idzko, M., Panther, E. \& Di Virgilio, F. (2006). The P2X7 receptor: a key player in IL-1 processing and release. J Immunol 176, 3877-83.

Filvaroff, E., Erlebacher, A., Ye, J., Gitelman, S. E., Lotz, J., Heillman, M. \& Derynck, R. (1999). Inhibition of TGF-beta receptor signaling in osteoblasts leads to decreased bone remodeling and increased trabecular bone mass. Development 126, 4267-79.

Fukui, T. (1993). Analysis of stress-strain curves in the rat molar periodontal ligament after application of orthodontic force. Am J Orthod Dentofacial Orthop 104, 27 35.

Furuya, K., Sokabe, M. \& Furuya, S. (2005). Characteristics of subepithelial fibroblasts as a mechano-sensor in the intestine: cell-shape-dependent ATP release and P2Y1 signaling. J Cell Sci 118, 3289-304.

Gartland, A., Hipskind, R. A., Gallagher, J. A. \& Bowler, W. B. (2001). Expression of a $\mathrm{P} 2 \mathrm{X} 7$ receptor by a subpopulation of human osteoblasts. J Bone Miner Res 16, 846-56.

Goldin, B. (1989). Labial root torque: effect on the maxilla and incisor root apex. Am $J$ Orthod Dentofacial Orthop 95, 208-19.

Goldson, L. \& Henrikson, C. O. (1975). Root resorption during Begg treatment; a longitudinal roentgenologic study. Am J Orthod 68, 55-66.

Goltzman, D. (2008) Studies on the mechanisms of the skeletal anabolic action of endogenous and exogenous parathyroid hormone. Arch Biochem Biophys 473, 218-24.

Graber, T. M., Vanarsdall, R. L. \& Vig, K. W. L. (2005). Orthodontics : current principles \& techniques, 4th edition. Elsevier Mosby, St. Louis, MO. 
Grahames, C. B., Michel, A. D., Chessell, I. P. \& Humphrey, P. P. (1999). Pharmacological characterization of ATP- and LPS-induced IL-1beta release in human monocytes. Br J Pharmacol 127, 1915-21.

Grygorczyk, R. \& Hanrahan, J. W. (1997). CFTR-independent ATP release from epithelial cells triggered by mechanical stimuli. Am J Physiol 272, C1058-66.

Gu, B. J., Sluyter, R., Skarratt, K. K., Shemon, A. N., Dao-Ung, L. P., Fuller, S. J., Barden, J. A., Clarke, A. L., Petrou, S. \& Wiley, J. S. (2004). An Arg307 to Gln polymorphism within the ATP-binding site causes loss of function of the human P2X7 receptor. J Biol Chem 279, 31287-95.

Gudipaty, L., Munetz, J., Verhoef, P. A. \& Dubyak, G. R. (2003). Essential role for Ca2+ in regulation of IL-1beta secretion by P2X7 nucleotide receptor in monocytes, macrophages, and HEK-293 cells. Am J Physiol Cell Physiol 285, C286-99.

Harris, E. F., Kineret, S. E. \& Tolley, E. A. (1997). A heritable component for external apical root resorption in patients treated orthodontically. Am J Orthod Dentofacial Orthop 111, 301-9.

Hartsfield JK Jr, E. E., Al-Qawasmi RA. (2004). GENETIC FACTORS IN EXTERNAL APICAL ROOT RESORPTION AND ORTHODONTIC TREATMENT. Crit Rev Oral Biol Med. 15, 115-122.

Hartsfield, J. K., Jr., Everett, E. T. \& Al-Qawasmi, R. A. (2004). Genetic Factors in External Apical Root Resorption and Orthodontic Treatment. Crit Rev Oral Biol Med 15, 115-122.

Hirao, M., Tamai, N., Tsumaki, N., Yoshikawa, H. \& Myoui, A. (2006). Oxygen tension regulates chondrocyte differentiation and function during endochondral ossification. J Biol Chem 281, 31079-92.

Hock, J. M., Canalis, E. \& Centrella, M. (1990). Transforming growth factor-beta stimulates bone matrix apposition and bone cell replication in cultured fetal rat calvariae. Endocrinology 126, 421-6.

Hollender, L., Ronnerman, A. \& Thilander, B. (1980). Root resorption, marginal bone support and clinical crown length in orthodontically treated patients. Eur J Orthod 2, 197-205.

Hou, Y., Liang, T. \& Luo, C. (1997). [Effects of IL-1 on experimental tooth movement in rabbits]. Zhonghua Kou Qiang Yi Xue Za Zhi 32, 46-8.

Hsieh, Y. F., Robling, A. G., Ambrosius, W. T., Burr, D. B. \& Turner, C. H. (2001). Mechanical loading of diaphyseal bone in vivo: the strain threshold for an osteogenic response varies with location. J Bone Miner Res 16, 2291-7.

Hurwitz, A., Finci-Yeheskel, Z., Milwidsky, A., Yagel, S., Adashi, E. Y., Laufer, N. \& Mayer, M. (1997). In-vitro modulation of plasminogen activator activity, prostaglandin E and nitric oxide production by interleukin-1 in pregnant mare serum gonadotrophin-primed theca-interstitial cells. Hum Reprod 12, 774-9.

Hwang, S. G., Yu, S. S., Poo, H. \& Chun, J. S. (2005). c-Jun/activator protein-1 mediates interleukin-1beta-induced dedifferentiation but not cyclooxygenase-2 expression in articular chondrocytes. J Biol Chem 280, 29780-7.

Ignelzi, M. A., Jr., Liu, Y. H., Maxson, R. E., Jr. \& Snead, M. L. (1995). Genetically engineered mice: tools to understand craniofacial development. Crit Rev Oral Biol Med 6, 181-201. 
Iwasaki, L. R., Gibson, C. S., Crouch, L. D., Marx, D. B., Pandey, J. P. \& Nickel, J. C. (2006). Speed of tooth movement is related to stress and IL-1 gene polymorphisms. Am J Orthod Dentofacial Orthop 130, 698 e1-9.

Iwasaki, L. R., Haack, J. E., Nickel, J. C., Reinhardt, R. A. \& Petro, T. M. (2001). Human interleukin-1 beta and interleukin-1 receptor antagonist secretion and velocity of tooth movement. Arch Oral Biol 46, 185-9.

Jager, A., Zhang, D., Kawarizadeh, A., Tolba, R., Braumann, B., Lossdorfer, S. \& Gotz, W. (2005). Soluble cytokine receptor treatment in experimental orthodontic tooth movement in the rat. Eur J Orthod 27, 1-11.

Jakobsson, R. \& Lind, V. (1973). Variation in root length of the permanent maxillary central incisor. Scand J Dent Res 81, 335-8.

Jilka, R. L. (2007). Molecular and cellular mechanisms of the anabolic effect of intermittent PTH. Bone 40, 1434-46.

John, K. \& Barakat, A. I. (2001). Modulation of ATP/ADP concentration at the endothelial surface by shear stress: effect of flow-induced ATP release. Ann Biomed Eng 29, 740-51.

Jones, M. L., Hickman, J., Middleton, J., Knox, J. \& Volp, C. (2001). A validated finite element method study of orthodontic tooth movement in the human subject. $J$ Orthod 28, 29-38.

Judex, S., Garman, R., Squire, M., Donahue, L. R. \& Rubin, C. (2004). Genetically based influences on the site-specific regulation of trabecular and cortical bone morphology. J Bone Miner Res 19, 600-6.

Kaku, M., Kohno, S., Kawata, T., Fujita, I., Tokimasa, C., Tsutsui, K. \& Tanne, K. (2001). Effects of vascular endothelial growth factor on osteoclast induction during tooth movement in mice. $J$ Dent Res 80, 1880-3.

Kale, S., Kocadereli, I., Atilla, P. \& Asan, E. (2004). Comparison of the effects of 1,25 dihydroxycholecalciferol and prostaglandin E2 on orthodontic tooth movement. Am J Orthod Dentofacial Orthop 125, 607-14.

Kanzaki, H., Chiba, M., Arai, K., Takahashi, I., Haruyama, N., Nishimura, M. \& Mitani, H. (2006). Local RANKL gene transfer to the periodontal tissue accelerates orthodontic tooth movement. Gene Ther 13, 678-85.

Kanzaki, H., Chiba, M., Takahashi, I., Haruyama, N., Nishimura, M. \& Mitani, H. (2004). Local OPG gene transfer to periodontal tissue inhibits orthodontic tooth movement. J Dent Res 83, 920-5.

Karst, M., Gorny, G., Galvin, R. J. \& Oursler, M. J. (2004). Roles of stromal cell RANKL, OPG, and M-CSF expression in biphasic TGF-beta regulation of osteoclast differentiation. J Cell Physiol 200, 99-106.

Katona, T. R. (1994). A Mechanical Engineering Analysis of Orthodontics Associated External Root Resorption. . In Biological Mechanisms of Tooth Eruption, Resorption and Replacement by Implants. (ed. Z. Davidovitch), pp. 537-543. EBSCO Media, Birmingham, AL.

Katona, T. R., Paydar, N. H., Akay, H. U. \& Roberts, W. E. (1995). Stress analysis of bone modeling response to rat molar orthodontics. J Biomech 28, 27-38.

Katsuragi, T. \& Migita, K. (2004). [The mechanism of ATP release as an autocrine/paracrine molecule]. Nippon Yakurigaku Zasshi 123, 382-8. 
Katz, S., Boland, R. \& Santillan, G. (2006). Modulation of ERK 1/2 and p38 MAPK signaling pathways by ATP in osteoblasts: Involvement of mechanical stressactivated calcium influx, PKC and Src activation. Int J Biochem Cell Biol.

Kawarizadeh, A., Bourauel, C., Gotz, W. \& Jager, A. (2003a). [Numerical study of tension and strain distribution around rat molars]. Biomed Tech (Berl) 48, 90-6.

Kawarizadeh, A., Bourauel, C. \& Jager, A. (2003b). Experimental and numerical determination of initial tooth mobility and material properties of the periodontal ligament in rat molar specimens. Eur J Orthod 25, 569-78.

Kawarizadeh, A., Bourauel, C., Zhang, D., Gotz, W. \& Jager, A. (2004). Correlation of stress and strain profiles and the distribution of osteoclastic cells induced by orthodontic loading in rat. Eur J Oral Sci 112, 140-7.

Ke, H. Z., Qi, H., Weidema, A. F., Zhang, Q., Panupinthu, N., Crawford, D. T., Grasser, W. A., Paralkar, V. M., Li, M., Audoly, L. P., Gabel, C. A., Jee, W. S., Dixon, S. J., Sims, S. M. \& Thompson, D. D. (2003). Deletion of the P2X7 nucleotide receptor reveals its regulatory roles in bone formation and resorption. Mol Endocrinol 17, 1356-67.

Kerkweg, U. \& de Groot, H. (2005). ATP-induced calcium increase as a potential first signal in mechanical tissue trauma. A laser scanning microscopic study on cultured mouse skeletal myocytes. Shock 24, 440-6.

Kim, I., Paik, K. S. \& Lee, S. P. (2005). Quantitative evaluation of the accuracy of microcomputed tomography in tooth measurement. Clin Anat.

Kim, M., Jiang, L. H., Wilson, H. L., North, R. A. \& Surprenant, A. (2001). Proteomic and functional evidence for a P2X7 receptor signalling complex. Embo $J \mathbf{2 0}$, 6347-58.

Kjaer, I. (1995). Morphological characteristics of dentitions developing excessive root resorption during orthodontic treatment. Eur J Orthod 17, 25-34.

Kohler, T., Beyeler, M., Webster, D. \& Muller, R. (2005). Compartmental bone morphometry in the mouse femur: reproducibility and resolution dependence of microtomographic measurements. Calcif Tissue Int 77, 281-90.

Kohno, S., Kaku, M., Kawata, T., Fujita, T., Tsutsui, K., Ohtani, J., Tenjo, K., Tohma, Y., Motokawa, M., Shigekawa, M., Kamada, H. \& Tanne, K. (2005). Neutralizing effects of an anti-vascular endothelial growth factor antibody on tooth movement. Angle Orthod 75, 797-804.

Kokkinos, P. P., Shaye, R., Alam, B. S. \& Alam, S. Q. (1993). Dietary lipids, prostaglandin E2 levels, and tooth movement in alveolar bone of rats. Calcif Tissue Int 53, 333-7.

Koseki, T., Gao, Y., Okahashi, N., Murase, Y., Tsujisawa, T., Sato, T., Yamato, K. \& Nishihara, T. (2002). Role of TGF-beta family in osteoclastogenesis induced by RANKL. Cell Signal 14, 31-6.

Krischak, G. D., Augat, P., Wachter, N. J., Kinzl, L. \& Claes, L. E. (1999). Predictive value of bone mineral density and Singh index for the in vitro mechanical properties of cancellous bone in the femoral head. Clin Biomech (Bristol, Avon) 14, 346-51.

Krishnan, V. (2005). Critical issues concerning root resorption: a contemporary review. World J Orthod 6, 30-40. 
Krishnan, V. \& Davidovitch, Z. (2006). Cellular, molecular, and tissue-level reactions to orthodontic force. Am J Orthod Dentofacial Orthop 129, 469 e1-32.

Kronenberg, H. M. (2003). Developmental regulation of the growth plate. Nature 423, 332-6.

Krstin, N., Dorow, C., Franke, R. P. \& Sander, F. G. (2002). [Experiments to determine the time dependent material properties of the periodontal ligament]. Biomed Tech (Berl) 47, 202-8.

Kubota, T., Yamauchi, M., Sasaguri, K., Ueda, R., Onozaki, J., Sato, S. \& Suzuki, Y. (1990). Regulation by transforming growth factor-beta (TGF-beta) in bone and periodontium. Bull Kanagawa Dent Coll 18, 143-9.

Kurol, J. \& Owman-Moll, P. (1998). Hyalinization and root resorption during early orthodontic tooth movement in adolescents. Angle Orthod 68, 161-5.

Kurol, J., Owman-Moll, P. \& Lundgren, D. (1996). Time-related root resorption after application of a controlled continuous orthodontic force. Am J Orthod Dentofacial Orthop 110, 303-10.

Labasi, J. M., Petrushova, N., Donovan, C., McCurdy, S., Lira, P., Payette, M. M., Brissette, W., Wicks, J. R., Audoly, L. \& Gabel, C. A. (2002). Absence of the $\mathrm{P} 2 \mathrm{X} 7$ receptor alters leukocyte function and attenuates an inflammatory response. J Immunol 168, 6436-45.

Lang, S. M., Moyle, D. D., Berg, E. W., Detorie, N., Gilpin, A. T., Pappas, N. J., Jr., Reynolds, J. C., Tkacik, M. \& Waldron, R. L., 2nd. (1988). Correlation of mechanical properties of vertebral trabecular bone with equivalent mineral density as measured by computed tomography. J Bone Joint Surg Am 70, 1531-8.

Lee, W. C. (1990). Experimental study of the effect of prostaglandin administration on tooth movement--with particular emphasis on the relationship to the method of PGE1 administration. Am J Orthod Dentofacial Orthop 98, 231-41.

Leiker, B. J., Nanda, R. S., Currier, G. F., Howes, R. I. \& Sinha, P. K. (1995). The effects of exogenous prostaglandins on orthodontic tooth movement in rats. Am J Orthod Dentofacial Orthop 108, 380-8.

Leitao, R. F., Ribeiro, R. A., Chaves, H. V., Rocha, F. A., Lima, V. \& Brito, G. A. (2005). Nitric oxide synthase inhibition prevents alveolar bone resorption in experimental periodontitis in rats. $J$ Periodontol 76, 956-63.

Leitao, R. F., Rocha, F. A., Chaves, H. V., Lima, V., Cunha, F. Q., Ribeiro, R. A. \& Brito, G. A. (2004). Locally applied isosorbide decreases bone resorption in experimental periodontitis in rats. J Periodontol 75, 1227-32.

Levander, E. \& Malmgren, O. (1988). Evaluation of the risk of root resorption during orthodontic treatment: a study of upper incisors. Eur J Orthod 10, 30-8.

Li, J., Liu, D., Ke, H. Z., Duncan, R. L. \& Turner, C. H. (2005). The P2X7 nucleotide receptor mediates skeletal mechanotransduction. J Biol Chem 280, 42952-9.

Lister, M. F., Sharkey, J., Sawatzky, D. A., Hodgkiss, J. P., Davidson, D. J., Rossi, A. G. \& Finlayson, K. (2007). The role of the purinergic P2X7 receptor in inflammation. J Inflamm (Lond) 4, 5.

Long, P., Liu, F., Piesco, N. P., Kapur, R. \& Agarwal, S. (2002). Signaling by mechanical strain involves transcriptional regulation of proinflammatory genes in human periodontal ligament cells in vitro. Bone 30, 547-52. 
Lossdorfer, S., Gotz, W. \& Jager, A. (2002). Localization of IL-1alpha, IL-1 RI, TNF, TNF-RI and TNF-RII during physiological drift of rat molar teeth--an immunohistochemical and in situ hybridization study. Cytokine 20, 7-16.

Lotz, J. C., Gerhart, T. N. \& Hayes, W. C. (1990). Mechanical properties of trabecular bone from the proximal femur: a quantitative CT study. J Comput Assist Tomogr 14, 107-14.

Lu, L. H., Lee, K., Imoto, S., Kyomen, S. \& Tanne, K. (1999). Histological and histochemical quantification of root resorption incident to the application of intrusive force to rat molars. Eur J Orthod 21, 57-63.

Lukic, I. K., Grcevic, D., Kovacic, N., Katavic, V., Ivcevic, S., Kalajzic, I. \& Marusic, A. (2005). Alteration of newly induced endochondral bone formation in adult mice without tumour necrosis factor receptor 1. Clin Exp Immunol 139, 236-44.

Lupi, J. E., Handelman, C. S. \& Sadowsky, C. (1996). Prevalence and severity of apical root resorption and alveolar bone loss in orthodontically treated adults. Am $J$ Orthod Dentofacial Orthop 109, 28-37.

Marseiller, E. (1977). [Les Dents Humaines], 8 edition. Gauthier-Villars, Paris.

Massey, H. M., Scopes, J., Horton, M. A. \& Flanagan, A. M. (2001). Transforming growth factor-beta1 (TGF-beta) stimulates the osteoclast-forming potential of peripheral blood hematopoietic precursors in a lymphocyte-rich microenvironment. Bone 28, 577-82.

McFadden, W. M., Engstrom, C., Engstrom, H. \& Anholm, J. M. (1989). A study of the relationship between incisor intrusion and root shortening. Am J Orthod Dentofacial Orthop 96, 390-6.

McLaughlin, K. (1964). Quantitative determination of root resorption during orthodontic treatment. Am J Orthod Dentofacial Orthop 50, 143.

Meikle, M. C. (2006). The tissue, cellular, and molecular regulation of orthodontic tooth movement: 100 years after Carl Sandstedt. Eur J Orthod 28, 221-40.

Melsen, B. (1999). Biological reaction of alveolar bone to orthodontic tooth movement. Angle Orthod 69, 151-8.

Melsen, B. (2001). Tissue reaction to orthodontic tooth movement--a new paradigm. Eur J Orthod 23, 671-81.

Middleton, J., Jones, M. \& Wilson, A. (1996). The role of the periodontal ligament in bone modeling: the initial development of a time-dependent finite element model. Am J Orthod Dentofacial Orthop 109, 155-62.

Milner, P., Bodin, P., Loesch, A. \& Burnstock, G. (1990a). Rapid release of endothelin and ATP from isolated aortic endothelial cells exposed to increased flow. Biochem Biophys Res Commun 170, 649-56.

Milner, P., Bodin, P., Loesch, A. \& Burnstock, G. (1992). Increased shear stress leads to differential release of endothelin and ATP from isolated endothelial cells from 4and 12-month-old male rabbit aorta. $J$ Vasc Res 29, 420-5.

Milner, P., Kirkpatrick, K. A., Ralevic, V., Toothill, V., Pearson, J. \& Burnstock, G. (1990b). Endothelial cells cultured from human umbilical vein release ATP, substance P and acetylcholine in response to increased flow. Proc Biol Sci 241, 245-8.

Mirabella, A. D. \& Artun, J. (1995a). Prevalence and severity of apical root resorption of maxillary anterior teeth in adult orthodontic patients. Eur J Orthod 17, 93-9. 
Mirabella, A. D. \& Artun, J. (1995b). Risk factors for apical root resorption of maxillary anterior teeth in adult orthodontic patients. Am J Orthod Dentofacial Orthop 108, 48-55.

Mitsui, N., Suzuki, N., Maeno, M., Mayahara, K., Yanagisawa, M., Otsuka, K. \& Shimizu, N. (2005). Optimal compressive force induces bone formation via increasing bone sialoprotein and prostaglandin E(2) production appropriately. Life Sci 77, 3168-82.

Miyoshi, K., Igarashi, K., Saeki, S., Shinoda, H. \& Mitani, H. (2001). Tooth movement and changes in periodontal tissue in response to orthodontic force in rats vary depending on the time of day the force is applied. Eur J Orthod 23, 329-38.

Mochizuki, H., Hakeda, Y., Wakatsuki, N., Usui, N., Akashi, S., Sato, T., Tanaka, K. \& Kumegawa, M. (1992). Insulin-like growth factor-I supports formation and activation of osteoclasts. Endocrinology 131, 1075-80.

Mundy, G. R. (1991). The effects of TGF-beta on bone. Ciba Found Symp 157, 137-43; discussion 143-51.

Myers, L. M., Brinkley, J.F. (1995). Visualization of brain surface features using registered partially segmented MRI scans. Proceedings SPIE Medical Imaging: Image Display., 43-52.

Naemsch, L. N., Dixon, S. J. \& Sims, S. M. (2001). Activity-dependent development of P2X7 current and Ca2+ entry in rabbit osteoclasts. J Biol Chem 276, 39107-14.

Nagerl, H., Burstone, C. J., Becker, B. \& Kubein-Messenburg, D. (1991). Centers of rotation with transverse forces: an experimental study. Am J Orthod Dentofacial Orthop 99, 337-45.

Nakamura, K., Sahara, N. \& Deguchi, T. (2001). Temporal changes in the distribution and number of macrophage-lineage cells in the periodontal membrane of the rat molar in response to experimental tooth movement. Arch Oral Biol 46, 593-607.

Natali, A., Pavan, P., Carniel, E. \& Dorow, C. (2004). Viscoelastic response of the periodontal ligament: an experimental-numerical analysis. Connect Tissue Res 45, 222-30.

Newman, W. G. (1975). Possible etiologic factors in external root resorption. Am J Orthod 67, 522-39.

Ngan, D. C., Kharbanda, O. P., Byloff, F. K. \& Darendeliler, M. A. (2004). The genetic contribution to orthodontic root resorption: a retrospective twin study. Aust Orthod J 20, 1-9.

Nishijima, Y., Yamaguchi, M., Kojima, T., Aihara, N., Nakajima, R. \& Kasai, K. (2006). Levels of RANKL and OPG in gingival crevicular fluid during orthodontic tooth movement and effect of compression force on releases from periodontal ligament cells in vitro. Orthod Craniofac Res 9, 63-70.

Norrdin, R. W., Jee, W. S. \& High, W. B. (1990). The role of prostaglandins in bone in vivo. Prostaglandins Leukot Essent Fatty Acids 41, 139-49.

North, R. A. (2002). Molecular physiology of P2X receptors. Physiol Rev 82, 1013-67.

North, R. A. \& Barnard, E. A. (1997). Nucleotide receptors. Curr Opin Neurobiol 7, 34657.

Ogasawara, T., Yoshimine, Y., Kiyoshima, T., Kobayashi, I., Matsuo, K., Akamine, A. \& Sakai, H. (2004). In situ expression of RANKL, RANK, osteoprotegerin and cytokines in osteoclasts of rat periodontal tissue. J Periodontal Res 39, 42-9. 
Ohata, H., Tanaka, K., Aizawa, H., Ao, Y., Iijima, T. \& Momose, K. (1997). Lysophosphatidic acid sensitises $\mathrm{Ca} 2+$ influx through mechanosensitive ion channels in cultured lens epithelial cells. Cell Signal 9, 609-16.

Olejniczak, A. J. \& Grine, F. E. (2006). Assessment of the accuracy of dental enamel thickness measurements using microfocal X-ray computed tomography. Anat Rec A Discov Mol Cell Evol Biol 288, 263-75.

Oshiro, T., Shiotani, A., Shibasaki, Y. \& Sasaki, T. (2002). Osteoclast induction in periodontal tissue during experimental movement of incisors in osteoprotegerindeficient mice. Anat Rec 266, 218-25.

Patel, A. S., Reigada, D., Mitchell, C. H., Bates, S. R., Margulies, S. S. \& Koval, M. (2005). Paracrine stimulation of surfactant secretion by extracellular ATP in response to mechanical deformation. Am J Physiol Lung Cell Mol Physiol 289, L489-96.

Peters, O. A., Laib, A., Ruegsegger, P. \& Barbakow, F. (2000). Three-dimensional analysis of root canal geometry by high-resolution computed tomography. J Dent Res 79, 1405-9.

Pilkington, M. F., Sims, S. M. \& Dixon, S. J. (2001). Transforming growth factor-beta induces osteoclast ruffling and chemotaxis: potential role in osteoclast recruitment. J Bone Miner Res 16, 1237-47.

Proffit, W. R. \& Fields, H. W. (2000). Contemporary orthodontics, 3rd edition. Mosby, St. Louis.

Provatidis, C. G. (1999). Numerical Estimation of the Centres of Rotation and Resistance in Orthodontic Tooth Movement. Comput Methods Biomech Biomed Engin 2, 149-156.

Provatidis, C. G. (2000). A comparative FEM-study of tooth mobility using isotropic and anisotropic models of the periodontal ligament. Finite Element Method. Med Eng Phys 22, 359-70.

Provatidis, C. G. (2002). Parametric finite element analysis and closed-form solutions in orthodontics. Comput Methods Biomech Biomed Engin 5, 101-12.

Qian, H., Chen, J. \& Katona, T. R. (2001). The influence of PDL principal fibers in a 3dimensional analysis of orthodontic tooth movement. Am J Orthod Dentofacial Orthop 120, 272-9.

Qu, Y., Franchi, L., Nunez, G. \& Dubyak, G. R. (2007). Nonclassical IL-1 beta secretion stimulated by $\mathrm{P} 2 \mathrm{X} 7$ receptors is dependent on inflammasome activation and correlated with exosome release in murine macrophages. J Immunol 179, 191325.

Rahimi, A., Keilig, L., Bendels, G., Klein, R., Buzug, T. M., Abdelgader, I., Abboud, M. \& Bourauel, C. (2005). 3D reconstruction of dental specimens from 2D histological images and microCT-scans. Comput Methods Biomech Biomed Engin 8, 167-76.

Raisz, L. G., Fall, P. M., Gabbitas, B. Y., McCarthy, T. L., Kream, B. E. \& Canalis, E. (1993a). Effects of prostaglandin E2 on bone formation in cultured fetal rat calvariae: role of insulin-like growth factor-I. Endocrinology 133, 1504-10.

Raisz, L. G., Pilbeam, C. C. \& Fall, P. M. (1993b). Prostaglandins: mechanisms of action and regulation of production in bone. Osteoporos Int 3 Suppl 1, 136-40. 
Ralston, S. H. \& Grabowski, P. S. (1996). Mechanisms of cytokine induced bone resorption: role of nitric oxide, cyclic guanosine monophosphate, and prostaglandins. Bone 19, 29-33.

Ramanathan, C. \& Hofman, Z. (2006). Root resorption in relation to orthodontic tooth movement. Acta Medica (Hradec Kralove) 49, 91-5.

Rassendren, F., Buell, G. N., Virginio, C., Collo, G., North, R. A. \& Surprenant, A. (1997). The permeabilizing ATP receptor, P2X7. Cloning and expression of a human cDNA. J Biol Chem 272, 5482-6.

Reitan, K. (1951). The initial tissue reaction incident to orthodontic tooth movement as related to the influence of function; an experimental histologic study on animal and human material. Acta Odontol Scand 6, 1-240.

Reitan, K. (1970). Evaluation of orthodontic forces as related to histologic and mechanical factors. SSO Schweiz Monatsschr Zahnheilkd 80, 579-96.

Reitan, K. (1994). Orthodontics- current principles and techniques. (ed. T. M. Graber, Vanarsdall RL). Mosby, St Louis.

Ren, Y., Maltha, J. C. \& Kuijpers-Jagtman, A. M. (2003). Optimum force magnitude for orthodontic tooth movement: a systematic literature review. Angle Orthod 73, 8692.

Roberts, W. E. (1999). Bone dynamics of osseointegration, ankylosis, and tooth movement. J Indiana Dent Assoc 78, 24-32.

Roberts, W. E. (2005). Bone physiology, metabolism and biomechanics in orthodontic practice In Orthodontics: Current Principles and Techniques. (ed. T. M. Graber). Mosby, St. Louis.

Roberts, W. E., Goodwin, W. C., Jr. \& Heiner, S. R. (1981). Cellular response to orthodontic force. Dent Clin North Am 25, 3-17.

Roberts, W. E., Huja, S., Roberts J.A. (2004). Bone modeling: biomechanics, molecular mechanisms and clinical perspectives. Seminars in Orthodontics 10,123-61.

Roberts, W. E., Smith, R. K., Zilberman, Y., Mozsary, P. G. \& Smith, R. S. (1984). Osseous adaptation to continuous loading of rigid endosseous implants. Am J Orthod 86, 95-111.

Robling, A. G., Castillo, A. B. \& Turner, C. H. (2006a). Biomechanical and molecular regulation of bone remodeling. Annu Rev Biomed Eng 8, 455-98.

Robling, A. G., Castillo, A. B. \& Turner, C. H. (2006b). Biomechanical and Molecular Regulation of Bone Remodeling. Annu Rev Biomed Eng.

Robling, A. G. \& Turner, C. H. (2002). Mechanotransduction in bone: genetic effects on mechanosensitivity in mice. Bone 31, 562-9.

Romanello, M., Pani, B., Bicego, M. \& D'Andrea, P. (2001). Mechanically induced ATP release from human osteoblastic cells. Biochem Biophys Res Commun 289, 1275 81.

Rubartelli, A. Lotze, M. T. (2007). Inside, outside, upside down: damage-associated molecular-pattern molecules (DAMPs) and redox. Trends Immunol 28, 429-36.

Rygh, P. (1974). Hyalinization of the periodontal ligament incident to orthodontic tooth movement. Nor Tannlaegeforen Tid 84, 352-7. 
Rygh, P. (1976). Ultrastructural changes in tension zones of rat molar periodontium incident to orthodontic tooth movement. Am J Orthod 70, 269-81.

Saito, M., Saito, S., Ngan, P. W., Shanfeld, J. \& Davidovitch, Z. (1991a). Interleukin 1 beta and prostaglandin $\mathrm{E}$ are involved in the response of periodontal cells to mechanical stress in vivo and in vitro. Am J Orthod Dentofacial Orthop 99, 22640.

Saito, S., Ngan, P., Rosol, T., Saito, M., Shimizu, H., Shinjo, N., Shanfeld, J. \& Davidovitch, Z. (1991b). Involvement of PGE synthesis in the effect of intermittent pressure and interleukin-1 beta on bone resorption. $J$ Dent Res 70, 2733.

Sameshima, G. T. \& Sinclair, P. M. (2001). Predicting and preventing root resorption: Part I. Diagnostic factors. Am J Orthod Dentofacial Orthop 119, 505-10.

Sauer, H., Hescheler, J. \& Wartenberg, M. (2000). Mechanical strain-induced Ca(2+) waves are propagated via ATP release and purinergic receptor activation. Am $J$ Physiol Cell Physiol 279, C295-307.

Schneider, E. M., Vorlaender, K., Ma, X., Du, W. \& Weiss, M. (2006). Role of ATP in trauma-associated cytokine release and apoptosis by P2X7 ion channel stimulation. Ann N Y Acad Sci 1090, 245-52.

Schwartz, A. (1932). Tissue changes incidental to orthodontic tooth movement. Int Journal Orthodontia 18, 331-352.

Schwarz, A. (1932). Tissue changes incident to orthodontic tooth movement. Int J Orthod 18, 331-52.

Seifi, M., Eslami, B. \& Saffar, A. S. (2003). The effect of prostaglandin E2 and calcium gluconate on orthodontic tooth movement and root resorption in rats. Eur $J$ Orthod 25, 199-204.

Sells Galvin, R. J., Gatlin, C. L., Horn, J. W. \& Fuson, T. R. (1999). TGF-beta enhances osteoclast differentiation in hematopoietic cell cultures stimulated with RANKL and M-CSF. Biochem Biophys Res Commun 265, 233-9.

Skarratt, K. K., Fuller, S. J., Sluyter, R., Dao-Ung, L. P., Gu, B. J. \& Wiley, J. S. (2005). A 5 ' intronic splice site polymorphism leads to a null allele of the P2X7 gene in 1$2 \%$ of the Caucasian population. FEBS Lett 579, $2675-8$.

Sluyter, R., Shemon, A. N. \& Wiley, J. S. (2004). Glu496 to Ala polymorphism in the P2X7 receptor impairs ATP-induced IL-1 beta release from human monocytes. $J$ Immunol 172, 3399-405.

Smith, R. J. \& Burstone, C. J. (1984). Mechanics of tooth movement. Am J Orthod 85, 294-307.

Solini, A., Chiozzi, P., Morelli, A., Fellin, R. \& Di Virgilio, F. (1999). Human primary fibroblasts in vitro express a purinergic P2X7 receptor coupled to ion fluxes, microvesicle formation and IL-6 release. J Cell Sci 112 ( Pt 3), 297-305.

Spencer, E. M., Liu, C. C., Si, E. C. \& Howard, G. A. (1991). In vivo actions of insulinlike growth factor-I (IGF-I) on bone formation and resorption in rats. Bone 12, 21-6.

Spielmann, T., Wieslander, L. \& Hefti, A. F. (1989). [Acceleration of orthodontically induced tooth movement through the local application of prostaglandin (PGE1)]. Schweiz Monatsschr Zahnmed 99, 162-5. 
Stubley, R. (1976). The influence of transseptal fibers on incisor position and diastema formation. Am J Orthod 70, 645-62.

Sun, W., Starly, B. Nam, J., Darling A. (2005). Bio-CAD modeling and its applications in computer-aided tissue engineering. Computer-Aided Design., 1097-114.

Surprenant, A., Rassendren, F., Kawashima, E., North, R. A. \& Buell, G. (1996). The cytolytic P2Z receptor for extracellular ATP identified as a P2X receptor (P2X7). Science 272, 735-8.

Taithongchai, R., Sookkorn, K. \& Killiany, D. M. (1996). Facial and dentoalveolar structure and the prediction of apical root shortening. Am J Orthod Dentofacial Orthop 110, 296-302.

Taniguchi, N., Yoshida, K., Ito, T., Tsuda, M., Mishima, Y., Furumatsu, T., Ronfani, L., Abeyama, K., Kawahara, K., Komiya, S., Maruyama, I., Lotz, M., Bianchi, M. E., Asahara, H. (2007) Stage-specific secretion of HMGB1 in cartilage regulates endochondral ossification. Mol Cell Biol 27, 5650-63.

Tanne, K., Burstone, C. J. \& Sakuda, M. (1989a). Biomechanical responses of tooth associated with different root lengths and alveolar bone heights: changes of stress distributions in the PDL. J Osaka Univ Dent Sch 29, 17-24.

Tanne, K., Koenig, H. A., Burstone, C. J. \& Sakuda, M. (1989b). Effect of moment to force ratios on stress patterns and levels in the PDL. J Osaka Univ Dent Sch 29, 916.

Tanne, K., Nagataki, T., Inoue, Y., Sakuda, M. \& Burstone, C. J. (1991). Patterns of initial tooth displacements associated with various root lengths and alveolar bone heights. Am J Orthod Dentofacial Orthop 100, 66-71.

Tanne, K., Sakuda, M. \& Burstone, C. J. (1987). Three-dimensional finite element analysis for stress in the periodontal tissue by orthodontic forces. Am J Orthod Dentofacial Orthop 92, 499-505.

Thaller, S. R., Hoyt, J., Tesluk, H. \& Holmes, R. (1993). Effect of insulin-like growth factor-1 on zygomatic arch bone regeneration: a preliminary histological and histometric study. Ann Plast Surg 31, 421-8.

Thomsen, J. S., Laib, A., Koller, B., Prohaska, S., Mosekilde, L. \& Gowin, W. (2005). Stereological measures of trabecular bone structure: comparison of 3D micro computed tomography with 2D histological sections in human proximal tibial bone biopsies. J Microsc 218, 171-9.

Thongudomporn, U. \& Freer, T. J. (1998). Anomalous dental morphology and root resorption during orthodontic treatment: a pilot study. Aust Orthod J 15, 162-7.

Thyagarajan, T., Totey, S., Danton, M. J. \& Kulkarni, A. B. (2003). Genetically altered mouse models: the good, the bad, and the ugly. Crit Rev Oral Biol Med 14, 15474.

Toms, S. R., Lemons, J. E., Bartolucci, A. A. \& Eberhardt, A. W. (2002). Nonlinear stress-strain behavior of periodontal ligament under orthodontic loading. Am J Orthod Dentofacial Orthop 122, 174-9.

Turner, J. T., Weisman, G. A., Landon, L. A., Park, M. \& Camden, J. M. (1998). Salivary gland nucleotide receptors: evidence for functional expression of both $\mathrm{P} 2 \mathrm{X}$ and P2Y subtypes. Eur J Morphol 36 Suppl, 170-5. 
van Steenbergen, E., Burstone, C. J., Prahl-Andersen, B. \& Aartman, I. H. (2005a). The influence of force magnitude on intrusion of the maxillary segment. Angle Orthod 75, 723-9.

van Steenbergen, E., Burstone, C. J., Prahl-Andersen, B. \& Aartman, I. H. (2005b). The relation between the point of force application and flaring of the anterior segment. Angle Orthod 75, 730-5.

Vanden Bulcke, M. M., Burstone, C. J., Sachdeva, R. C. \& Dermaut, L. R. (1987). Location of the centers of resistance for anterior teeth during retraction using the laser reflection technique. Am J Orthod Dentofacial Orthop 91, 375-84.

Vanden Bulcke, M. M., Dermaut, L. R., Sachdeva, R. C. \& Burstone, C. J. (1986). The center of resistance of anterior teeth during intrusion using the laser reflection technique and holographic interferometry. Am J Orthod Dentofacial Orthop 90, 211-20.

Verna, C. \& Melsen, B. (2003). Tissue reaction to orthodontic tooth movement in different bone turnover conditions. Orthod Craniofac Res 6, 155-63.

Verna, C., Zaffe, D. \& Siciliani, G. (1999). Histomorphometric study of bone reactions during orthodontic tooth movement in rats. Bone 24, 371-9.

Viecilli, R., Katona, T. \& Roberts, W. (2007). Optimization of microCT data processing for modelling of dental structures in orthodontic studies. Comput Methods Biomech Biomed Engin 10, 257-63.

Viecilli, R. F. (2006). Self-corrective T-loop design for differential space closure. Am J Orthod Dentofacial Orthop 129, 48-53.

Viecilli, R. F., Chen, J., Katona, T. R. \& Roberts, W. E. (In press). The force system of an adjustable molar root movement mechanism. Am J Orthod Dentofacial Orthop.

Viecilli, R. F., Katona, T. R., Chen, J., Hartsfield Jr, J. K. \& Roberts, W. E. (2008). The Mechanical Environment of Orthodontic Tooth Movement and Root Resorption. Am J Orthod Dentofacial Orthop In press.

Viecilli, R. F., T.R., K., Chen, J., Roberts, W. E. \& Hartsfield Jr., J. K. (Submitted in 2008). Comparison of Dentoalveolar Morphology in C57B/6 and P2X7R KO mice for the Development of Biomechanical Orthodontic Models. Anat Rec.

Vollmer, D., Bourauel, C., Maier, K. \& Jager, A. (1999). Determination of the centre of resistance in an upper human canine and idealized tooth model. Eur J Orthod 21, 633-48.

von Bohl, M., Maltha, J. C., Von Den Hoff, J. W. \& Kuijpers-Jagtman, A. M. (2004). Focal hyalinization during experimental tooth movement in beagle dogs. Am J Orthod Dentofacial Orthop 125, 615-23.

Waarsing, J. H., Day, J. S. \& Weinans, H. (2004). An improved segmentation method for in vivo microCT imaging. J Bone Miner Res 19, 1640-50.

Wachter, N. J., Augat, P., Krischak, G. D., Sarkar, M. R., Mentzel, M., Kinzl, L. \& Claes, L. (2001). Prediction of strength of cortical bone in vitro by microcomputed tomography. Clin Biomech (Bristol, Avon) 16, 252-6.

Wachter, N. J., Krischak, G. D., Mentzel, M., Sarkar, M. R., Ebinger, T., Kinzl, L., Claes, L. \& Augat, P. (2002). Correlation of bone mineral density with strength and microstructural parameters of cortical bone in vitro. Bone 31, 90-5.

Waldman, H. B. (1998). Changing number and distribution of orthodontists: 1987-1995. Am J Orthod Dentofacial Orthop 114, 50-4. 
Wiley, J. S., Dao-Ung, L. P., Li, C., Shemon, A. N., Gu, B. J., Smart, M. L., Fuller, S. J., Barden, J. A., Petrou, S. \& Sluyter, R. (2003). An Ile-568 to Asn polymorphism prevents normal trafficking and function of the human P2X7 receptor. $J$ Biol Chem 278, 17108-13.

Wilson, H. L., Francis, S. E., Dower, S. K. \& Crossman, D. C. (2004). Secretion of intracellular IL-1 receptor antagonist (type 1) is dependent on P2X7 receptor activation. J Immunol 173, 1202-8.

Wise, G. E., Lin, F. \& Zhao, L. (1995). Immunolocalization of interleukin-1 alpha in rat mandibular molars and its enhancement after in vivo injection of epidermal growth factor. Cell Tissue Res 280, 21-6.

Wright, K. W. \& Yettram, A. L. (1979). An analytical investigation into possible mechanical causes of bone remodelling. J Biomed Eng 1, 41-9.

Xu, L. X., Kukita, T., Yu, H., Nakano, Y. \& Koga, T. (1998). Expression of the mRNA for types I and II interleukin-1 receptors in dental tissues of mice during tooth development. Calcif Tissue Int 63, 351-6.

Yamaguchi, M., Aihara, N., Kojima, T. \& Kasai, K. (2006). RANKL Increase in Compressed Periodontal Ligament Cells from Root Resorption. J Dent Res 85, 751-6.

Yamaguchi, M. \& Kasai, K. (2005). Inflammation in periodontal tissues in response to mechanical forces. Arch Immunol Ther Exp (Warsz) 53, 388-98.

Yamaguchi, M., Ozawa, Y., Nogimura, A., Aihara, N., Kojima, T., Hirayama, Y. \& Kasai, K. (2004). Cathepsins B and L increased during response of periodontal ligament cells to mechanical stress in vitro. Connect Tissue Res 45, 181-9.

Yamamoto, K., Sokabe, T., Ohura, N., Nakatsuka, H., Kamiya, A. \& Ando, J. (2003). Endogenously released ATP mediates shear stress-induced Ca2+ influx into pulmonary artery endothelial cells. Am J Physiol Heart Circ Physiol 285, H793803.

Yamasaki, K., Shibasaki, Y. \& Fukuhara, T. (1983). [Use of prostaglandins in orthodontic tooth movement--experimental and clinical studies]. Shikai Tenbo 62, 480-92.

Yamasaki, K., Shibata, Y. \& Fukuhara, T. (1982). The effect of prostaglandins on experimental tooth movement in monkeys (Macaca fuscata). J Dent Res 61, 14446.

Yamasaki, K., Shibata, Y., Imai, S., Tani, Y., Shibasaki, Y. \& Fukuhara, T. (1984). Clinical application of prostaglandin E1 (PGE1) upon orthodontic tooth movement. Am J Orthod 85, 508-18.

Yan, T., Riggs, B. L., Boyle, W. J. \& Khosla, S. (2001). Regulation of osteoclastogenesis and RANK expression by TGF-beta1. J Cell Biochem 83, 320-5.

Yang, M., Ding, Y., Xu, R. \& Zhou, B. (2000). [Distribution of IL-1 beta in periodontium of experimental osteoporosis rats during orthodontic tooth movement]. Hua Xi Kou Qiang Yi Xue Za Zhi 18, 314-6.

Yee, J. A. (1979). Response of periodontal ligament cells to orthodontic force: ultrastructural identification of proliferating fibroblasts. Anat Rec 194, 603-14.

Yee, J. A., Kimmel, D. B. \& Jee, W. S. (1976). Periodontal ligament cell kinetics following orthodontic tooth movement. Cell Tissue Kinet 9, 293-302. 
Yeni, Y. N., Christopherson, G. T., Dong, X. N., Kim, D. G. \& Fyhrie, D. P. (2005). Effect of microcomputed tomography voxel size on the finite element model accuracy for human cancellous bone. J Biomech Eng 127, 1-8.

Yoshida, H., Hayashi, S., Kunisada, T., Ogawa, M., Nishikawa, S., Okamura, H., Sudo, T., Shultz, L. D. \& Nishikawa, S. (1990). The murine mutation osteopetrosis is in the coding region of the macrophage colony stimulating factor gene. Nature 345 , 442-4.

Zengo, A. N., Bassett, C. A., Pawluk, R. J. \& Prountzos, G. (1974). In vivo bioelectric petentials in the dentoalveolar complex. Am J Orthod 66, 130-9.

Zengo, A. N., Pawluk, R. J. \& Bassett, C. A. (1973). Stress-induced bioelectric potentials in the dentoalveolar complex. Am J Orthod 64, 17-27.

Zhang, D. (1992). [The experimental research on the changes in the amount of prostaglandins in rat alveolar bone during tooth movement]. Zhonghua Kou Qiang Yi Xue Za Zhi 27, 290-2, 319. 


\section{CURRICULUM VITAE}

\section{Rodrigo F. Viecilli}

\section{Education:}

Indiana University

MSc

2008-

Indiana University

$\mathrm{PhD}$

2004- 2009

Hospital de Aeronautica de Canoas

Orthodontics Specialization

2001-2003

Universidade Federal do RS

Dentistry

1996-2000

Instituto Tecnologico de Aeronautica Aeronautical Engineering (Inc)

1994-1996

\section{Awards:}

$1^{\text {st }}$ Place- American Association of Orthodontists Milo Hellman Award for Basic Science Research (2009)

$1^{\text {st }}$ Place- Trident Award for Innovation in Oral Care (2008)

$2^{\text {nd }}$ Place- American Association of Orthodontists Charles Schultz Award for Basic

Science Research (2008)

$2^{\text {nd }}$ Place- Maynard K. Hine Manuscript Award (2008)

\section{Publications:}

Viecilli, R.F. (2006) "Self-corrective t-loop for differential space closure.” Am J Orthod Dentofac Orthop. 129(1):48-53.

Viecilli, R.F., T.R. Katona and W. E. Roberts (2007). "Optimization of microCT data processing for modelling of dental structures in orthodontic studies.” Comput Methods Biomech Biomed Engin 10(4):257-63.

Martins R.P., P.H. Buschang, R.F. Viecilli, A. dos Santos-Pinto (2008). "Curvature versus v-bends in a group B titanium T-loop spring.” Angle Orthodontist. 78(3):517-23.

Viecilli, R. F., T. R. Katona, J. Chen, J. K. Hartsfield, Jr. and W. E. Roberts (2008). “The three-dimensional mechanical environment of orthodontic tooth movement and root resorption.” Am J Orthod Dentofacial Orthop. . 133(6):791.e11-26

Viecilli, R.F., J. Chen, T. R. Katona and W. E. Roberts (In press). "The force system generated by an adjustable molar root movement mechanism.” Am J Orthod Dentofac Orthop.

Viecilli, R. F., T. R. Katona, J. Chen, J. K. Hartsfield and W. E. Roberts (In press). "Comparison of dentoalveolar morphology in C57B/6 and P2X7R KO mice for the development of biomechanical orthodontic models.” Anat Rec.

Viecilli, R. F., T. R. Katona, J. Chen, J. K. Hartsfield, Jr. and W. E. Roberts (Accepted). "Orthodontic mechanotransduction and the role of the P2X7 receptor." Am J Orthod Dentofac Orthop. 